Review Article

\title{
Neuroprotective Phytochemicals in Experimental Ischemic Stroke: Mechanisms and Potential Clinical Applications
}

\author{
Hui Xu $\mathbb{D}^{1,2}$ Emily Wang, ${ }^{3}$ Feng Chen, ${ }^{1}$ Jianbo Xiao $\mathbb{D}^{4},{ }^{4}$ and Mingfu Wang $\mathbb{D}^{1,2}$ \\ ${ }^{1}$ Institute for Advanced Study, Shenzhen University, Shenzhen 508060, China \\ ${ }^{2}$ School of Biological Sciences, The University of Hong Kong, Pokfulam Road, Hong Kong, China \\ ${ }^{3}$ Rice University, Houston, Texas, USA \\ ${ }^{4}$ International Research Center for Food Nutrition and Safety, Jiangsu University, Zhenjiang 212013, China
}

Correspondence should be addressed to Jianbo Xiao; jianboxiao@yahoo.com and Mingfu Wang; mfwang@hku.hk

Received 22 December 2020; Revised 10 March 2021; Accepted 29 March 2021; Published 29 April 2021

Academic Editor: Daniele Vergara

Copyright (C) 2021 Hui Xu et al. This is an open access article distributed under the Creative Commons Attribution License, which permits unrestricted use, distribution, and reproduction in any medium, provided the original work is properly cited.

\begin{abstract}
Ischemic stroke is a challenging disease with high mortality and disability rates, causing a great economic and social burden worldwide. During ischemic stroke, ionic imbalance and excitotoxicity, oxidative stress, and inflammation are developed in a relatively certain order, which then activate the cell death pathways directly or indirectly via the promotion of organelle dysfunction. Neuroprotection, a therapy that is aimed at inhibiting this damaging cascade, is therefore an important therapeutic strategy for ischemic stroke. Notably, phytochemicals showed great neuroprotective potential in preclinical research via various strategies including modulation of calcium levels and antiexcitotoxicity, antioxidation, anti-inflammation and BBB protection, mitochondrial protection and antiapoptosis, autophagy/mitophagy regulation, and regulation of neurotrophin release. In this review, we summarize the research works that report the neuroprotective activity of phytochemicals in the past 10 years and discuss the neuroprotective mechanisms and potential clinical applications of 148 phytochemicals that belong to the categories of flavonoids, stilbenoids, other phenols, terpenoids, and alkaloids. Among them, scutellarin, pinocembrin, puerarin, hydroxysafflor yellow A, salvianolic acids, rosmarinic acid, borneol, bilobalide, ginkgolides, ginsenoside Rd, and vinpocetine show great potential in clinical ischemic stroke treatment. This review will serve as a powerful reference for the screening of phytochemicals with potential clinical applications in ischemic stroke or the synthesis of new neuroprotective agents that take phytochemicals as leading compounds.
\end{abstract}

\section{Introduction: Ischemic Stroke}

Stroke occurs when the blood supply to the brain tissue is interrupted or reduced. Generally, stroke can be divided into two major categories: ischemic stroke and hemorrhagic stroke, according to how the blood flow is disrupted. Ischemic stroke is caused by the occlusion of cerebral arteries by thrombi or embolisms, blocking the blood flow to one part of the brain. Hemorrhagic stroke results from the ruptures of a weakened blood vessel, leading to the accumulation of blood in the surrounding brain tissue [1]. Of the two, ischemic stroke is the primary type, accounting for about $80 \%$ of all strokes [2]. Stroke ranks second in the cause of death worldwide, and about 5.5 million people die from stroke each year (WHO health statistics). Besides, stroke has a high disability rate, resulting in permanent disability for around $50 \%$ of its survivors [3]. Many risk factors are associated with stroke, such as age, hypertension, obesity, hyperlipidemia, diabetes, smoking, and alcohol consumption. With the great increase in the aging population, the occurrence of stroke is predicted to continue rising, and the mortality of stroke may exceed $12 \%$ by 2030 [4]. Hence, stroke is a challenging disease that greatly increases the worldwide economic and social burden.

1.1. Pathophysiology of Ischemic Stroke. When ischemic stroke occurs, blood flow to the specific territory of the brain that is supplied by the occluded artery is reduced. Generally, 
the ischemic area of the brain can be divided into the infarct core and the ischemic penumbra according to the severity of the blood flow reduction. The infarct core is characterized by a rapid decrease in ATP levels and energy stores and severe ionic disruption, which result in cell death within a few minutes. Surrounding the core area is the ischemic penumbra. In this area, blood flow reduction is less severe due to perfusion from collateral blood vessels. Hence, the insult to the ischemic penumbra is much milder than that to the infarct core. As a result, multiple milder cell death mechanisms occur in this area such as inflammation and apoptosis, providing promising therapeutic targets for ischemic stroke [5]. Notably, the ischemic penumbra is dynamic, in which the infarct core expands at the cost of the penumbra during cerebral ischemia. Hereby, early reperfusion is the most effective manner to reduce the cerebral infarction of ischemic stroke patients [6].

Ischemic stroke injuries include two parts: ischemic injury and reperfusion injury. The cell death mechanisms of the ischemic brain are redundant, and at least three dominant mechanisms are involved: ionic imbalance and excitotoxicity, oxidative/nitrosative stress, and inflammation. Notably, those mechanisms are developed in a relatively certain order and become the dominant events at different stages of ischemic stroke. Generally, ionic imbalance and excitotoxicity play a critical role in the ischemic phase, and oxidative/nitrosative stress peaks at the beginning phase of reperfusion, while inflammation lasts for several days or weeks after reperfusion. After activation, those mechanisms affect the function of cell membranes and organelles such as the mitochondria, endoplasmic reticulum (ER), lysosomes, and nuclei. Consequently, different cell death pathways are activated, including apoptosis and necrosis [5]. Autophagy/mitophagy is also activated in ischemic stroke, but whether it promotes or decreases the cerebral ischemia-reperfusion $(I / R)$ injuries has not been agreed upon at present. Studies suggested that apoptosis and cytoprotective autophagy/mitophagy tended to be induced by moderate cerebral I/R injuries, while necrosis or destructive autophagy/mitophagy was activated during severe I/R damage [7]. The major mechanisms of cell death in ischemic stroke are illustrated in Figure 1.

1.2. Major Pharmacological Therapies for Ischemic Stroke. Major approaches to treat ischemic stroke can be divided into two types: recanalization and neuroprotection. Recanalization is aimed at restoring the blood flow with thrombolytic agents or accessory devices in the acute phase of ischemic stroke (from minutes to hours) or preventing the reoccurrence of stroke with antiplatelet and anticoagulant agents, while neuroprotection is aimed at protecting neurons from the different pathological factors of ischemic stroke [8]. Recently, researchers also pronounced the theory of promoting brain neurogenesis to achieve long-term recovery after ischemic stroke. Several compounds are found to enhance neurogenesis in experimental stroke models, such as epigallocatechin-3-gallate (EGCG), curcumin, and ginkgolide $\mathrm{K}$ [9-11]. Yet, no agents are clinically approved for this therapy at present.
1.2.1. Thrombolysis. Intravenous (IV) thrombolysis with recombination tissue plasminogen activator ( $\mathrm{r}$-tPA, alteplase) is the only US Food and Drug Administration(FDA-) approved pharmacological treatment for acute ischemic stroke [12]. tPA promotes the conversion of plasminogen to plasmin, an active proteolytic enzyme that cleaves the cross-linkages between fibrin molecules of clots [13]. Notably, r-tPA has a very short therapeutic window and is best when administrated within $3 \mathrm{~h}$ after symptom onset. Patients can still benefit from r-tPA when it is administrated between 3 and $4.5 \mathrm{~h}$ after cerebral ischemia. However, r-tPA is not recommended for patients whose treatment cannot be initiated within $4.5 \mathrm{~h}$ because it will greatly increase the rate of intracranial hemorrhage and neuronal excitotoxicity [14]. Clinically, the short therapeutic window drastically limits the eligible patients and only about $15 \%$ of the hospitalized patients are treated with r-tPA [14].

1.2.2. Antiplatelets and Anticoagulants. Antiplatelet and anticoagulant therapies are aimed at preventing the reoccurrence of stroke via the prevention of clot formation. Antiplatelets inhibit platelet activation or aggregation, while anticoagulants suppress the functions of clot-forming factors such as factors II, VII, and X. The common antiplatelet agents include aspirin, clopidogrel, dipyridamole, tirofiban, and eptifibatide. Clinical studies show that the risk of early recurrent stroke is decreased by aspirin administration within $48 \mathrm{~h}$ of ischemic stroke onset [15]. As for anticoagulants such as heparin, warfarin, dabigatran, rivaroxaban, and apixaban, it is found that urgent therapeutic anticoagulation benefits high-risk cardioembolic stroke patients. Yet, the use of anticoagulants may lead to symptomatic intracranial hemorrhage in unselected ischemic stroke patients [13].

1.2.3. Neuroprotection. Neuroprotective agents could reduce ischemic brain injuries via the promotion of neuronal survival, neuroplasticity, synaptogenesis, and neurogenesis. Hence, neuroprotection therapy could be combined with thrombolytic agents to reduce the second injuries of reperfusion [16]. Over the past two decades, over 1000 potential neuroprotective agents were found in experimental ischemic stroke, with nearly 200 agents having undergone clinical trials [17]. Particularly, edaravone and Dl-3-n-butylphthalide show great efficacy in clinical treatment and have been approved for ischemic stroke treatment in Japan and China, respectively.

Edaravone, with the trade name Radicut/Radicava, is a medication developed by Mitsubishi Tanabe Pharma of Japan. Edaravone is a free radical scavenger that targets peroxyl radicals. It was approved for the treatment of ischemic stroke in 2001 and amyotrophic lateral sclerosis (ALS) in 2017 [18]. Edaravone is widely applied in Japan, China, and other Asian countries, and nearly half of ischemic stroke patients receive edaravone treatment in Japan [19]. Clinical studies show that the combination of edaravone and intravenous thrombolysis therapy improves the neurological outcome of ischemic stroke patients $[19,20]$. Besides, edaravone is also found to reduce in-hospital mortality and 

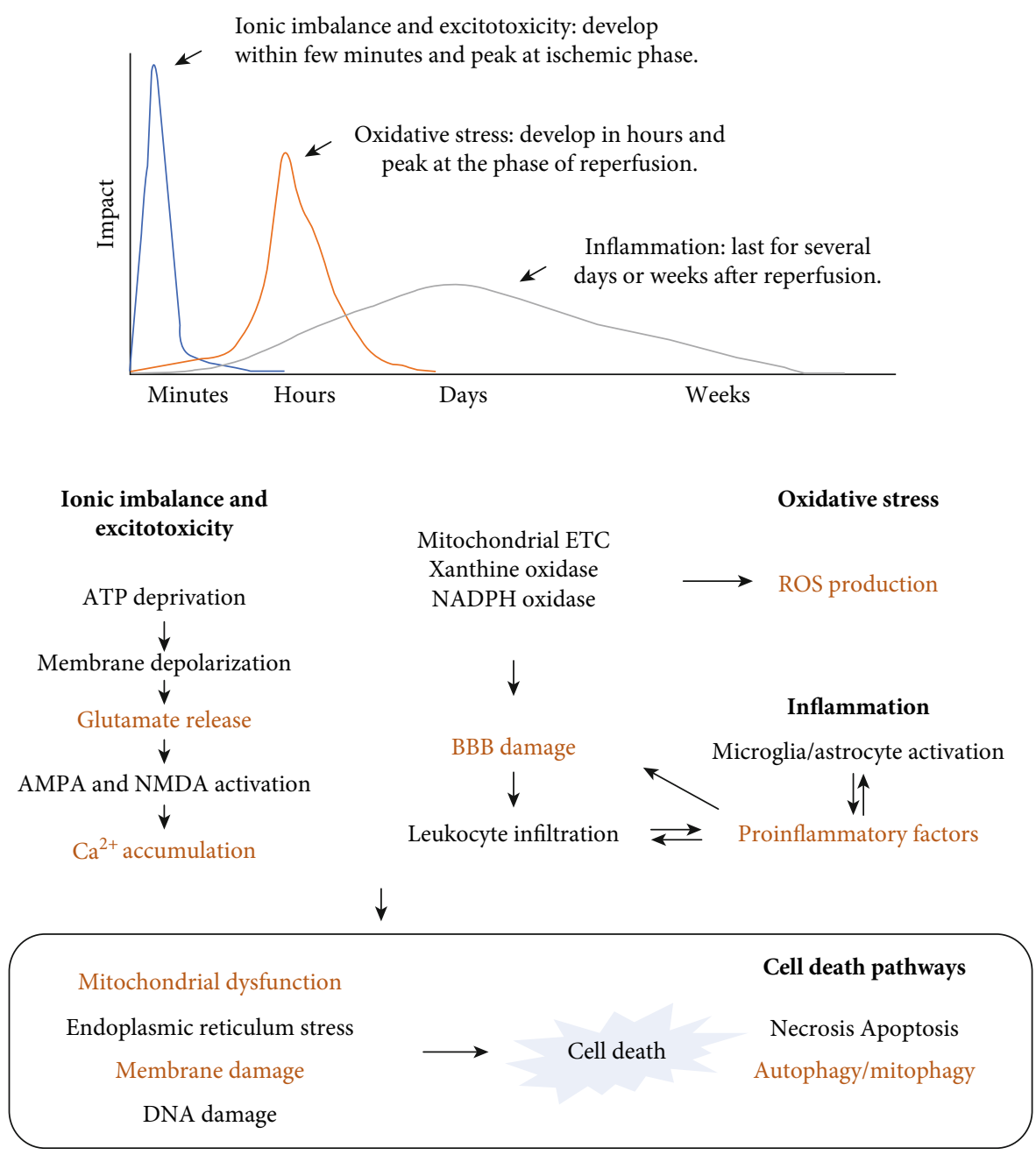

FIGURE 1: Dominant cell death mechanisms in ischemic stroke. Ionic imbalance and excitotoxicity, oxidative stress, and inflammation are major causes that lead to brain cell death in ischemic stroke. Ionic imbalance and excitotoxicity are developed within few minutes after ischemia and are the leading cause of cell death during the ischemic phase. Oxidative stress peaks at the beginning phase of reperfusion due to the sharply increased ROS production after oxygen restoration, while inflammation can last for several days or weeks after reperfusion contributing to the delayed cell death after ischemic stroke. Generally, these mechanisms can activate various cell death pathways such as necrosis, apoptosis, and autophagy/mitophagy directly or indirectly by promoting the dysfunction of organelles such as the mitochondria and endoplasmic reticulum.

intracranial hemorrhage when combined with endovascular thrombolysis therapy [21].

Dl-3-n-butylphthalide (NBP) is a neuroprotective drug developed by CSPC Pharmaceutical Group Limited. NBP is originally extracted from the seeds of Apium graveolens; synthesized NBP was later approved for ischemic stroke treatment in 2002. Clinically, NBP soft capsules and injections have been used to treat mild to moderate ischemic stroke patients in China. NBP is a multitargeted agent, exerting neuroprotection in ischemic stroke via antioxidation, anti-inflammation, antiapoptosis, and mitochondrial protection [22]. Clinical studies indicate that NBP improves neurological deficits such as waking, speaking, sense, thought, and memory impairments, promoting long-term recovery of ischemic stroke patients [23].

\subsection{Common Models for Experimental Ischemic Stroke Research}

1.3.1. Middle Cerebral Artery Occlusion Model. Most ischemic strokes occur in the middle cerebral artery (MCA) territory of the human brain, so animal models are developed to induce ischemia in this area to mimic the clinical situation. There are several ways to occlude the MCA in experimental ischemic stroke research, and the most commonly used one is the intraluminal suture MCA occlusion (MCAO) model. In this model, a monofilament is inserted into the internal carotid artery (ICA) and advanced to the origin of MCA to block the blood flow. The monofilament can be left in the blood vessel to mimic the permanent ischemia (pMCAO) or pulled out to achieve reperfusion as a model of transient focal cerebral ischemia (tMCAO/R). Normally, 60-120 min of ischemia is commonly used in rats to induce neuronal death and cerebral infarction. In addition, MCA can also be occluded directly by clipping, ligation, or hooks through the craniectomy [2]. Robinson et al. firstly report an approach that can achieve direct occlusion of the distal MCA 
(dMCAO) through ligation in Sprague-Dawley rats. The dMCAO model is more reproducible than the suture MCAO model, but it may induce skull trauma, resulting in cortical inflammation and spreading depression $[24,25]$.

1.3.2. Photothrombotic Model. The photothrombotic stroke model is induced by the intravascular photooxidation of a photosensitive dye (e.g., Rose Bengal). For stroke induction, the photosensitive dye is intravenously or intraperitoneally injected, after which the targeted cerebral vessel is illuminated with a light beam of a specific wavelength through the intact skull to activate the dye. The activated dye then promotes endothelial injuries and platelet aggregation via the formation of superoxides. Notably, the application of stereotactic coordinates during illumination makes it possible to induce infarction at the desired cortical brain region. Due to its high reproducibility and low mortality, this model is often used to study the longterm functional outcomes after stroke. Yet, the photothrombotic model has fundamental discrepancies with the pathophysiology of human ischemic stroke because of the lack of the ischemic penumbra and collateral blood flow [25-27].

1.3.3. Thromboembolic Clot Model. The thromboembolic model involves the application of prepared blood clots to achieve focal cerebral vascular occlusion. The clots are usually formed spontaneously or induced by thrombin from autologous blood. Besides, injection of thrombin directly to the MCA or intracranial segment of ICA is also a common method to induce clots. This model has a high similarity to the mechanism of vascular occlusion in human ischemic stroke, so it is often used to study thrombolysis or mechanical reperfusion-related strategies [28]. For example, $\mathrm{Ma}$ et al. reported the effect of pinocembrin in extending the therapeutic window of r-tPA with this model [29]. However, the infarct location and size induced by the thromboembolic model are variable due to differences in the size and elasticity of clots. Hence, this model is less reproducible than the MCAO model [25].

1.3.4. Global Cerebral Ischemia Model. Global cerebral ischemia is aimed at blocking all the blood flow to the brain, causing neuronal injuries to the selectively vulnerable brain areas such as the CA1 pyramidal neurons of the hippocampus and neocortex. There are many ways to achieve global cerebral ischemia including decapitation, neck tourniquet, ventricular fibrillation, and occlusion of ICAs and vertebral vessels. Currently, the most used method is bilateral ICA occlusion, namely, the two-vessel occlusion (2-VO) model. Notably, the 2-VO model induces cerebral injuries in the vulnerable brain areas with a very short ischemia period. It is found that damage can be observed in the hippocampus of animals that only suffer from $2 \mathrm{~min}$ of bilateral ICA occlusion. Although the global cerebral ischemia model is not fully compliant with the pathogenesis of human ischemic stroke, it still has advantages in studying the poststroke cognitive and neurological outcomes due to its selective damage to the vulnerable hippocampus [30].

\section{Neuroprotective Strategies of Phytochemicals in Experimental Ischemic Stroke}

Dominant mechanisms that lead to cell death in ischemic stroke include ionic imbalance and excitotoxicity, oxidative stress, and inflammation. After initiation, these events then activate various cell death pathways, including necrosis, apoptosis, and autophagy/mitophagy, directly or indirectly by causing the dysfunction of organelles, such as mitochondria and ER. Theoretically, all the events in this damaging cascade could be modulated to achieve potential neuroprotection in ischemic stroke. Notably, several strategies have been proved to be effective in experimental ischemic stroke, and the major strategies that are modulated by phytochemicals are reviewed in this section.

2.1. Calcium Modulation and Antiexcitotoxicity. Glucose and oxygen deprivation disrupts the electron transport chain (ETC), limiting the production of ATP in mitochondria. ATP depletion then enhances the anaerobic metabolism, inducing disorder of $\mathrm{Na}^{+} / \mathrm{K}^{+}$-ATPase and $\mathrm{Ca}^{2+} / \mathrm{H}^{+}$-ATPase pumps. As a result, the intracellular $\mathrm{H}^{+}, \mathrm{Na}^{+}$, and $\mathrm{Ca}^{2+}$ levels are greatly elevated, causing neuronal cell membrane depolarization and acidosis [5]. Membrane depolarization markedly elevates the release of excitatory amino acids such as glutamate. Meanwhile, the reuptake of those excitatory amino acids is impaired due to energy failure. Hence, glutamate is excessively accumulated in the extracellular space, leading to the activation of two glutamate-dependent $\mathrm{Ca}^{2+}$ ion channels: NMDA (N-methyl-D-aspartate) and AMPA ( $\alpha$-amino-3-hydroxy-5-methyl-4-isoxazolepropionic acid) receptors [31]. Consequently, intracellular $\mathrm{Ca}^{2+}$ is dramatically elevated, activating many $\mathrm{Ca}^{2+}$-dependent enzymes to promote necrotic and apoptotic cell death [32]. Accordingly, inhibition of intracellular $\mathrm{Ca}^{2+}$ accumulation or extracellular glutamate levels would reduce neuronal damage. In addition, ionic imbalance and excitotoxicity peak at the end of ischemia, so agents that target this strategy should be administrated as early as possible. Late administration could lead to ineffectiveness or even damage to brain tissues. Detailed strategies for calcium modulation and antiexcitotoxicity include enhancing the reuptake of glutamate, upregulating the inhibitory amino acid systems, modulating the activity of NMDA receptors, and regulating the non-glutamatedependent calcium-permeable cation channels.

2.1.1. Enhancing the Reuptake of Glutamate. Reuptake of glutamate is mediated by the excitatory amino acid transporters (EAATs) in astrocytes and neurons. Three types of EAATs are found in the central nervous system (CNS) of rodents, including GLAST (glutamate/aspartate transporter), GLT-1 (glutamate transporter-1), and EAAC1. Under ischemia conditions, functions of EAATs are suppressed due to ionic imbalance and ATP depletion, enhancing the neurotoxicity of glutamate. Hence, upregulation of the expression or activity of EAATs helps to reduce excitotoxicity in ischemic stroke [33]. Notably, the effectiveness of EAAT modulation has been indicated by many in vitro and in vivo studies. Several 
EAAT activators such as ginsenoside Rb1 and harmine have been found to possess neuroprotective activities in experimental ischemic stroke $[34,35]$.

2.1.2. Upregulating the Inhibitory Amino Acid Systems. Inhibitory amino acids could bind to their corresponding receptors and inhibit the postsynaptic excitatory response, and the major inhibitory amino acid in the CNS is gammaaminobutyric acid (GABA). Cerebral ischemia not only disrupts the balance between glutamate and GABA release but also suppresses the activity of GABA receptors. As a result, the inhibitory effect of GABA is markedly inhibited in ischemic stroke. Hereby, improving the glutamate and GABA balance or upregulating GABA receptors contributes to brain repair. As evidence, the GABA receptor agonist clomethiazole was reported to exert neuroprotection in animal models [36]. Besides, EGCG and ginkgolide B, two natural products that mediated neuroprotection, were found to be achieved partially by improving the balance of excitatory/inhibitory amino acids $[37,38]$.

\subsubsection{Modulating the Activity of NMDA Receptors. NMDA} receptors consist of four subunits: two GluN1 and two GluN2 (glutamate-binding). Early studies regarded the NMDA receptor as a vital regulator for glutamate-mediated neurotoxicity in ischemic stroke. Hence, numerous NMDA receptor antagonists were tested to evaluate their neuroprotective activities. However, researchers found that the toxicities of NMDA receptor antagonists were high, limiting their further application. Recently, studies indicated that the high toxicity of NMDA receptor antagonists might be attributed to the dual function of NMDA receptors in ischemic stroke. It is found that the functions of NMDA receptors depend on their locations and the subunit types. Generally, the GluN2 subunit greatly affects the function of NMDA receptors. GluN2A is mainly expressed at the synapse and promotes cell survival by activating prosurvival pathways such as PI3K (phosphoinositide 3-kinase)/Akt (protein kinase B) and CREB (cyclic AMP response element-binding protein). On the contrary, GluN2B is highly expressed in extrasynaptic sites and activates prodeath pathways such as nNOS (neuronal nitric oxide synthases). During cerebral ischemia, GluN2B is the primary activated NMDA receptor, contributing to cerebral I/R injuries. Hence, selectively inhibiting GluN2B or its downstream prodeath pathways would be a neuroprotective strategy [36, 39]. For example, TatNR2B9c, a peptide that inhibited GluN2B-mediated prodeath pathways, was found to protect neurons in MCAO models [40]. In addition, upregulation of GluN2A was reported to contribute to neuronal survival. As evidence, geniposide enhanced the expression of GluN2A and reduced brain damage in $\mathrm{tMCAO} / \mathrm{R}$ rats [41].

\subsubsection{Regulating the Non-Glutamate-Dependent Calcium-} Permeable Cation Channels. The influx of $\mathrm{Ca}^{2+}$ is also modulated via non-glutamate-dependent cation channels, including TRP (transient receptor potential) channels and ASICs (acid-sensing ion channels) [42]. TRP channels can be divided into six subgroups, with TRPC6, TRPM7, and TRPV1 being extensively studied in ischemic stroke. The roles of TRPs in ischemic stroke are different. TRPM7 and TRPV1 promote neuronal death by elevating the intracellular $\mathrm{Ca}^{2+}$ level. Yet, TRPC6 contributes to neuronal survival via activation of CaMK (calmodulin-dependent protein kinase) and CREB signaling pathways. Notably, cerebral ischemia promotes the expression of TRPM7 and TRPV1 and enhances the degradation of TRPC6. Hereby, upregulation of TRPC6 or downregulation of TRPM7/TRPV1 would decrease cerebral I/R-induced injuries [42-44]. As an example, TRPC6 was activated by resveratrol and calycosin in MCAO models [43, 45], while inhibition of TRPM7 and TRPV1 was observed in carvacrol- and capsaicin-mediated neuroprotection, respectively $[46,47]$.

ASICs, especially ASIC1a and ASIC2a, are found to mediate cerebral I/R-induced injuries. Among them, ASIC1a promotes $\mathrm{Ca}^{2+}$ influx and neuronal injuries after being activated by the increased acidosis during ischemia, while ASIC2a reduces brain damage as observed in a transient global ischemia model [48, 49]. Modulation of ASICs was observed in ginsenoside Rd-mediated neuroprotection, which inhibited ASIC1a and enhanced ASIC2a expression in $\mathrm{MCAO} / \mathrm{R}$ rats.

2.2. Antioxidation. Free radicals start to be produced during ischemia, surging in the reperfusion period, in which the free radical production systems such as mitochondrial ETC and enzymatic conversion systems are greatly promoted after oxygen restoration. In mitochondria, excessive $\mathrm{Ca}^{2+}$ accumulation during ischemia leads to the dephosphorylation of the oxidative phosphorylation (OxPhos) complexes, hyperactivating the ETC system. After reperfusion, the hyperactive ETC markedly promotes the generation of reactive oxygen species (ROS) with the supply of sufficient oxygen and glucose [50]. The enzymatic systems mainly include xanthine oxidase and NADPH (nicotinamide adenine dinucleotide phosphate) oxidase. Similarly, those enzymes are also hyperactivated during ischemia via accumulation, phosphorylation, or uncoupling, so ROS production in enzymatic systems is markedly enhanced after reperfusion [7].

Oxidative stress plays a critical role in reperfusion injuries. Firstly, oxidative stress directly destroys the cellular membrane system and DNA, leading to necrotic and apoptotic cell death. Secondly, oxidative stress enhances the opening of mPTP (mitochondrial permeability transition pore) in mitochondria, increasing the release of many proapoptotic factors such as cytochrome $\mathrm{c}$ and AIF (apoptosis-inducing factor). Thirdly, oxidative stress increases the permeability of the blood-brain barrier (BBB) by activating matrix metalloproteases (MMPs), thus elevating the incidence of cerebral hemorrhage, brain edema, and leukocyte infiltration $[51,52]$. Finally, oxidative stress interacts with the cascade of inflammation, further deteriorating reperfusion injuries. Accordingly, antioxidation would be an important strategy to reduce cerebral $\mathrm{I} / \mathrm{R}$ injuries.

Methods to modulate the oxidative stress in ischemic stroke are relatively uncomplicated, mainly including reducing NADPH oxidase-mediated ROS production and enhancing the antioxidant defense by activating the Nrf2 (nuclear 
factor erythroid 2-related factor 2) pathway. Yet, this strategy is found to be modulated by numerous neuroprotective agents including some phytochemicals.

\subsubsection{Reducing NADPH Oxidase-Mediated ROS Production.} NADPH oxidase (NOX) is regarded as the primary target to modulate ROS production in ischemic stroke, in which it is relatively hard to pharmacologically inhibit the ETC. NOXs have several homologs, with NOX2 and NOX4 playing critical roles in ischemic stroke. After ischemia, NOX4 expression in neurons is markedly increased, while NOX2 upregulation is mainly found in endothelial cells. Elevated NOX promotes the generation of ROS, so inhibition of NOX would help to reduce cerebral I/R-induced oxidative stress. The effectiveness of NOX inhibition is reported in animal models. To illustrate, NOX inhibitors, such as isoquercetin, ginsenoside Rb1, picroside II, and andrographolide, were all found to protect brain tissues from cerebral I/R damage [53-57].

\subsubsection{Enhancing the Antioxidant Defense via the Nrf2} Pathway. Normally, ROS could be scavenged by the intracellular antioxidant defenses, including enzymatic antioxidants (e.g., SOD and catalase) and nonenzymatic antioxidants (e.g., ascorbic acid) to maintain redox homeostasis [58]. However, cerebral I/R injuries greatly promote ROS production, overburdening the antioxidant defense systems. Hereby, strengthening the antioxidant defense is a critical strategy to reduce oxidative stress in ischemic stroke. $\mathrm{Nrf} 2$ is the major transcriptional factor that regulates the intracellular antioxidant defense, especially under stress conditions. Once activated, Nrf2 enhances the expression of various antioxidant enzymes such as GCL (glutamatecysteine ligase), HO-1 (heme oxygenase-1), and NQO1 $(\mathrm{NAD}(\mathrm{P}) \mathrm{H}$ dehydrogenase [quinone] 1). Overwhelming evidence indicates that $\mathrm{Nrf} 2$ reduces cerebral I/R-induced oxidative stress. Nrf2 activators such as sulforaphane, tert-butylhydroquinone, nobiletin, naringenin, astragaloside IV, and neferine were all reported to exert neuroprotection in experimental ischemic stroke [59-63].

2.3. Anti-Inflammation and BBB Protection. Inflammation is the primary poststroke damage that produces the delayed progression of cell death after ischemic stroke, developing and lasting for several days or weeks after reperfusion. Inflammation is jointly mediated by the infiltrated leukocytes and brain resident immune cells: microglia/macrophages and astrocytes. Under cerebral I/R conditions, microglia are activated rapidly and display two phenotypes: the proinflammatory phenotype (M1) and the anti-inflammatory phenotype (M2). M1 microglia contribute to neuronal cell death via secreting the proinflammatory cytokines, such as IL- $1 \beta$, IL6 , and TNF- $\alpha$. Yet, M2 microglia promote the recovery of the injured brain via anti-inflammatory mediators, such as IL-4, IL-10, and neurotrophins [64]. Astrocytes are activated after microglia and release multiple proinflammatory cytokines and inducible NOS (iNOS) after activation [65]. Then, the activated microglia and astrocytes promote the expression of adhesion molecules such as ICAM-1 (intercellular adhesion molecule 1) and induce the leukocyte infiltration into the ischemic brain, triggering a stronger cascade of inflammation. Worse even, the elevated level of proinflammatory factors activates MMPs and increases the BBB permeability. As a result, leukocyte infiltration is further elevated, creating a vicious cycle [66].

The inflammation cascade involves multiple regulators, so many targets could be modulated during this process. Methods to achieve anti-inflammation in experimental ischemic stroke mainly include regulation of microglial/astrocyte activation and leukocyte infiltration, inhibition of arachidonic acid release and metabolism, modulation of the transcriptional factors related to inflammation, and suppression of the TLR signaling pathway.

2.3.1. Regulation of Microglial/Astrocyte Activation and Leukocyte Infiltration. As discussed above, the activated microglia/astrocytes and infiltrated leukocytes greatly promoted the release of various proinflammatory factors, such as TNF- $\alpha$, IL-6, IL- $1 \beta$, MCP-1 (monocyte chemoattractant protein-1), ICAM-1, and iNOS, so inhibition of microglia1/astrocyte activation and leukocyte infiltration would contribute to neuroprotection. Accordingly, numerous neuroprotective agents were reported to modulate this strategy in ischemic stroke. To illustrate, scutellarin, epicatechin, fisetin, and calycosin were found to inhibit microglial activation in animal models of ischemic stroke, with epicatechin and fisetin also suppressing leukocyte infiltration [67-70]. Besides, salidroside-, ginkgolide B-, and celastrol-mediated neuroprotection were associated with the promotion of M2 microglial polarization, that is, transferring proinflammatory M1 microglia to antiinflammatory M2 microglia [71-73]. Furthermore, berberine, harmine, and tanshinone IIA exerted neuroprotection via inhibition of astrocyte activation [35, 74, 75].

\subsubsection{Inhibition of Arachidonic Acid Release and Metabolism.} Arachidonic acid (AA), a polyunsaturated fatty acid, is stored in the phospholipid membrane in the form of glycerol under normal conditions. Yet, elevated free radicals during ischemic stroke initiate the hydrolysis of phospholipid via activation of the phospholipases (PL, mainly PLA2 in the case of ischemic stroke). As a result, AA is released to the intracellular space and then degraded to produce several proinflammatory metabolites [76]. The degradation of AA is mediated by three independent enzymes: cyclooxygenases (COX) to form prostaglandins (PG), lipoxygenases (LOX) to form leukotrienes, and cytochrome $\mathrm{P}_{450}$ epoxygenases to form epoxyeicosatrienoic acids (EETs), respectively. Among them, COX-2 and 5-LOX are well studied in cerebral I/R-induced inflammation. It is shown that expressions of COX-2 and 5-LOX are increased after cerebral ischemia, and inhibition of COX-2 or 5-LOX by their corresponding inhibitors reduces brain damage in animal models. In addition, 12/15-LOX is also reported to promote cerebral ischemic injuries, as evidenced by few recent studies. Hence, many targets can be modulated in the metabolism of AA including PLA2, COX2, 5-LOX, and 12/15-LOX [65]. For instance, apigenin-, chrysin-, and picroside II-mediated neuroprotection were 
related to the suppression of COX-2 [77-79]. Besides, the 5LOX inhibitors caffeic acid and boswellic acid and 12/15LOX inhibitors baicalein and oxymatrine were reported to reduce cerebral damage in MCAO models [80-83].

\subsubsection{Modulation of the Transcriptional Factors Related to} Inflammation. A series of transcriptional factors participate in the cascade of inflammation, such as STAT3 (signal transducer and activator of transcription 3 ), NF- $\kappa \mathrm{B}$ (nuclear factor- $\kappa \mathrm{B}$ ), PPAR $\alpha$ (peroxisome proliferatoractivated receptor $\alpha$ ), and $\operatorname{PPAR} \gamma$. These transcriptional factors target diverse genes and eventually exert different functions in inflammation [84]. NF- $\kappa \mathrm{B}$ is a well-known proinflammatory transcriptional factor. After activation, NF- $\kappa \mathrm{B}$ promotes the expressions of various proinflammatory factors, such as iNOS, 5-LOX, COX-2, TNF- $\alpha$, and IL-6. Accordingly, inhibition of the activity of NF- $\kappa \mathrm{B}$ is found to reduce the cerebral infarction of MCAO rodents [84]. As an example, the neuroprotective activities of nobiletin and naringenin were mediated by inhibition of NF- $\kappa \mathrm{B}[60,85]$.

JAK2 (Janus kinase 2) is a receptor of proinflammatory cytokines, such as IL-6. Once activated, JAK2 promotes the phosphorylation and nuclear translocation of STAT3, initiating the expression of its target genes. The JAK2/STAT3 pathway is found to play dual roles in ischemic stroke. Some studies reported that the JAK2/STAT3 pathway contributes to brain recovery by promoting neuronal survival and neurogenesis. Yet, JAK2/STAT3 is also found to promote inflammation, especially when activated in the microglia [84]. Hence, several agents, such as atractylenolide III and sinomenine, were reported to suppress inflammation and reduce brain injuries via inhibition of the JAK2/STAT3 pathway in preclinical studies $[86,87]$.

PPARs are the major regulator of cellular glucose and lipid metabolism. Recent studies found that $\operatorname{PPAR} \alpha / \gamma$ agonists exhibit anti-inflammatory activities, indicating that PPAR $\alpha / \gamma$ might also mediate the inflammatory response. $\operatorname{PPAR} \alpha / \gamma$ are reported to suppress the inflammation cascade in ischemic stroke [84]. Accordingly, a PPAR $\gamma$ activator, malibatol A, and a $\operatorname{PPAR} \alpha / \gamma$ activator, icariin, were found to decrease cerebral damage in tMCAO/R models $[88,89]$.

2.3.4. Suppression of the TLR Signaling Pathway. TLR (Toll-like receptor), a transmembrane protein, can initiate inflammation in response to exogenous or endogenous stress. TLRs have several homologs, and TLR2/4 are reported to be involved in the inflammation cascade of ischemic stroke. The activation of TLR2/4 requires endogenous ligands, such as HMGB1 (high mobility group box 1), HSPs (heat shock proteins), hyaluronic acid, and fibronectin. After combination with ligands, the configuration of TLRs is changed, leading to the recruitment of its adaptors such as MyD88 (myeloid differentiation primary response 88 ) and TRIF (TIR-domain-containing adapter-inducing interferon- $\beta$ ). The recruited adaptors then promote inflammation via activation of NF- $\kappa \mathrm{B}$ [90]. Hence, inhibition of the HMGB1/TLR/MyD88/NF- $\kappa$ B pathway could be a potential neuroprotective strategy. Many neuroprotective compounds are reported to modulate this pathway. For instance, glycyrrhizin and berberine inhibited the HMGB1/TLR4 pathway, and vinpocetine suppressed the TLR4/MyD88/NF- $\kappa$ B signaling [91-93]. Beyond that, baicalin-, luteolin-, and curcumin-mediated neuroprotection were also found to be associated with the inhibition of TLRs [94-96].

2.4. Mitochondrial Protection and Antiapoptosis. It is known that mitochondria play a vital role in reperfusion-induced injury via the generation of excessive ROS [50]. Elevated intracellular ROS and $\mathrm{Ca}^{2+}$ levels lead to the opening of mPTP, a complicated complex existing in the mitochondrial membrane. As a result, the permeability of mitochondria is enhanced and many mitochondrial proapoptotic factors are released such as cytochrome $c$ and AIF [97]. Cytochrome c is a central regulator in caspase-dependent apoptosis. Released cytochrome c promotes the cascade of apoptosis via activation of caspase-9 and caspase-3. AIF is found to mainly mediate caspase-independent apoptosis. After release, AIF is translocated to the nucleus, binds to DNA, and promotes the chromatin condensation and annexin staining, initiating the apoptotic cascade [6].

Since the opening of $\mathrm{MPTP}$ is the major initiator for apoptosis, inhibition of the mPTP opening would be an effective neuroprotective strategy. Accordingly, hydroxysafflor yellow A-, gallic acid-, and picroside II-mediated neuroprotection were all found to be related to the inhibition of mPTP [98-100]. In addition, some regulators can modulate the opening of $\mathrm{mPTP}$ such as Bcl-2 family proteins and cyclophilin D. Bcl-2 proteins consist of proapoptotic proteins (e.g., Bax, Bad) and antiapoptotic proteins (e.g., $\mathrm{Bcl}-2, \mathrm{Bcl}-\mathrm{xl})$. It is found that Bax promotes mPTP formation, while $\mathrm{Bcl}-2$ could combine with Bax to inhibit its function. Hence, the ratio of $\mathrm{Bcl}-2 / \mathrm{Bax}$ is regarded as an important indicator of the mPTP opening, and many neuroprotective agents are found to regulate $\mathrm{Bcl}-2 / \mathrm{Bax}$ [97]. As an example, the ratio of $\mathrm{Bcl}-2 / \mathrm{Bax}$ was increased in galangin-treated pMCAO rats [101]. As for the other regulator, cyclophilin $\mathrm{D}$ promotes $\mathrm{MPTP}$ formation via binding to one of its components, the VDAC (voltage-dependent anion channel). Hereby, inhibition of cyclophilin D was also observed in the neuroprotective activities of some agents such as cyclosporin $\mathrm{A}$ and gallic acid [99]. The PI3K/Akt signaling pathway is a critical regulator of apoptosis. It is found that Akt promotes the phosphorylation of Bad, an inhibitor of Bcl-2. After phosphorylation, Bad separates from $\mathrm{Bcl}-2$ and promotes the binding of $\mathrm{Bcl}-2$ with mitochondria, suppressing the MPTP opening and subsequent cytochrome $c$ release [102]. Since the PI3K/Akt signaling pathway is fundamental in cerebral I/R-induced apoptosis, it is modulated by most of the antiapoptotic agents in experimental ischemic stroke. For example, puerarin- and silibinin (silybin)-mediated neuroprotection were associated with the upregulation of the PI3K/Akt signaling pathway $[103,104]$.

2.5. Autophagy/Mitophagy Regulation. Autophagy is a complicated process that transports the cytoplasmic proteins or organelles to lysosomes for degradation. The process of 
autophagy can be divided into four main steps: initiation, prolongation, fusion, and degradation. Initiation is aimed at forming the phagophore via the ULK1-initiated cascades. Prolongation is extending and closing of the phagophore to form a matured autophagosome that contains the targeted proteins or organelles. This process is mediated by the ATG12 and LC3 ubiquitin-like conjugation systems, in which LC3 II plays central roles. The mature autophagosome is then fused with the lysosome and degraded by lysosomal enzymes $[105,106]$. Autophagy is initially regarded as a nonselective process, but now it is widely accepted that autophagy can also be induced by a selective manner, such as through selective degradation of damaged mitochondria (mitophagy). The mitophagy cascade is similar to autophagy, except that it needs to detect the damaged mitochondria first. Generally, mitochondria which possess a decreased mitochondrial membrane potential $\left(\Delta \psi_{\mathrm{m}}\right)$ are identified and divided into two parts: healthy mitochondria and depolarized mitochondria. The depolarized mitochondria then initiate the mitophagy cascade and eventually are degraded. Notably, it is found that mitophagy is initiated after mitochondrial fission; that is, inhibition of mitochondrial fission will accordingly suppress the mitophagy [107].

Overwhelming evidence shows that autophagy/mitophagy is activated in various ischemic stroke models. Yet, the role of autophagy/mitophagy in cerebral I/R-induced injuries is still controversial at present. Several studies regard autophagy/mitophagy as a type of cell death, playing a detrimental role in ischemic stroke. Those studies indicate that neuronal death or brain damage is reduced after blocking the autophagy/mitophagy cascades via administration of 3-methyladenine (3-MA, an autophagosome formation inhibitor) or after knockdown of Beclin1 and Atg7, two critical regulators in the autophagy cascade in various in vitro and in vivo models [108]. Accordingly, inhibition of autophagy/mitophagy confers the neuroprotection of several agents, such as baicalein, calycosin, and puerarin [70, 109, 110].

On the contrary, autophagy/mitophagy is also found to play an important role in maintaining cellular homeostasis via degradation of defective or aggregated proteins and organelles [111]. The protective effects of autophagy/mitophagy in ischemic stroke are indicated by many investigations. For instance, Rami reported that inhibition of autophagy/mitophagy by 3-MA (3-methyladenine) or Atg7 knockdown in the reperfusion phase enhanced cytochrome c release and apoptosis both in vitro and in vivo [112]. In addition, many neuroprotective agents are reported to enhance the autophagy/mitophagy cascade in experimental ischemic stroke. To illustrate, the neuroprotective effects of triptolide, astragaloside IV, and ginsenoside Rb1 were found to be mediated by enhanced autophagy [113-115]. Besides, elevated mitophagy contributed to the neuroprotection of rapamycin, methylene blue, melatonin, and ginsenoside Rg1 in MCAO models [116, 117].

The controversial results are attributed to the differences in drug administration time points, doses, or routes [111]. Although no consensus has been reached at present, autophagy/mitophagy modulation is still considered to be a promising neuroprotective strategy due to its extensive interactions with the other cell death pathways such as necrosis and apoptosis. Yet, more studies are needed to further clarify its role in ischemic stroke.

2.6. Regulation of Neurotrophin Release. Neurotrophins are critical regulators of neuronal survival, development, function, and regeneration. There are many types of neurotrophins in the mammalian CNS, with NGF (nerve growth factor) and BDNF (brain-derived neurotrophic factor) being intensively studied in ischemic stroke. NGF is abundantly expressed in both the hippocampus and the cortex. After release, it binds to the TrkA (tropomyosin-related kinase A) receptor and triggers the activation of the Erk (extracellular signal-regulated kinase)/CREB pathway to improve neuronal recovery. BDNF is the most abundant neurotrophin in the mammalian CNS. BDNF binds to the TrkB receptor, activating several prosurvival pathways including PI3K/Akt signaling and MAPKs (mitogen-activated protein kinases). As one of the self-rescuing mechanisms for neurons, the expression of NGF/TrkA and BDNF/TrkB is upregulated after ischemic stroke $[118,119]$. Hence, the promotion of this process would contribute to neuronal survival and recovery. For instance, rutin and astaxanthin were found to upregulate the expression of NGF or BDNF and reduced cerebral infarction and neurological deficits in MCAO models $[119,120]$. In addition, some studies also evaluated the effects of exogenous neurotrophins in ischemic stroke. It is found that administration of exogenous neurotrophins exerted neuroprotection in animal models but failed to do the same in the clinical trials due to their low BBB permeability [121].

\section{Phytochemicals That Exert Neuroprotection in Experimental Ischemic Stroke}

Phytochemicals are the secondary metabolites of plants, such as vegetables, fruits, and herbs. Generally, phytochemicals can be divided into several chemical groups, including phenolics, terpenoids, and alkaloids. Phenolics are a class of compounds that possess at least one aromatic ring, with one or more hydroxyl groups attached. They can be further classified as flavonoids, stilbenes, phenolic acids, phenolic alcohols, and lignans. Terpenoids refer to the compounds that have the isoprene unit as their basic component, while alkaloids possess one or more nitrogen atoms in the heterocyclic ring [122]. Phytochemicals are famous for their antioxidative and anti-inflammatory activities, and some phytochemicals can usually act on more than one target to regulate cellular function. Chen et al. recently proposed a theory that the one-drug-multitarget strategy is more effective for ischemic stroke treatment when considering the complexity of stroke pathophysiology [123]. Hence, phytochemicals may have great potential in ischemic stroke treatment. In this section, we review the recent 10 years of research that reported the neuroprotective effects of phytochemicals in ischemic stroke. Only the phytochemicals that were tested on animal models of ischemic stroke are listed, and the ones that were studied 
extensively or possessed great translational potential are further discussed.

3.1. Flavonoids. Flavonoids include six major subgroups: flavones, flavanones, flavanols, flavonols, isoflavones, and anthocyanidins. In addition, flavonoids also largely exist in plants as glucoside derivatives, with the O-glycosidic bonds formed with different carbohydrates such as D-glucose, Dglucuronic acid, and D-galactose [124]. Totally, 46 kinds of neuroprotective flavonoids were found after searching the recent 10 years of studies in PubMed with keywords "Flavonoids, Stroke, Neuroprotection." The neuroprotective flavonoids and their functional mechanisms are listed in Table 1, and the chemical structures of the extensively studied flavonoids are shown in Table 2.

3.1.1. Flavones. Neuroprotective flavones include apigenin $[77,125]$, apigenin-7-O- $\beta$-D- $\left(-6^{\prime \prime}\right.$-p-coumaroyl)-glucopyranoside (APG) [126], vitexin [127], baicalein [82, 109], baicalin $[94,128-131]$, chrysin $[78,132,133]$, diosmin [134], ginkgetin [135], hispidulin [136], luteolin [95, 137, 138], luteoloside [139], orientin [140], nobiletin [60, 141-143], scutellarin [67, 144-147], and tricin 7-glucoside [148].

Scutellaria baicalensis is a traditional Chinese medicine that has long been used to treat ischemic stroke and cerebral edema [128]. Baicalein and baicalin are two principal components extracted from its roots. Baicalein was reported to improve cerebral infarction, brain edema, and neurobehavioral deficits in both the transient and permanent MCAO models [82, 109]. The neuroprotective strategies of baicalein mainly involve anti-inflammation, antiapoptosis, and antiautophagy. Cui et al. found that baicalein inhibited the 12/15LOX/p38/cPLA2 pathway and thus reduced arachidonic acid release to inhibit inflammation [82]. Besides, baicalein also suppressed the activation of NF- $\kappa \mathrm{B}$, providing another target for its anti-inflammatory activity [109]. Baicalin is also extensively studied in experimental ischemic stroke and found to improve cerebral infarction and poststroke cognitive impairments in different animal models. The most reported neuroprotective mechanism of baicalin was anti-inflammation, which was achieved via inhibition of the TLR2/4/NF- $\kappa \mathrm{B}$ pathway $[94,129]$. In addition, baicalin-mediated neuroprotection was also related to its antioxidant effect. Xu et al. reported that baicalin possessed a marked ability to scavenge peroxynitrite and reduce peroxynitrite-induced neuronal injuries [128]. Furthermore, baicalin was observed to show neuroprotection in a diabetic $\mathrm{MCAO} / \mathrm{R}$ rat model via activation of AMPK $\alpha$ - ( $5^{\prime}$ AMP-activated protein kinase $\alpha$-) mediated mitochondrial protection [130]. Notably, baicalin had the ability to cross the $\mathrm{BBB}$, reaching its peak concentration of $344 \mu \mathrm{g} / \mathrm{L}$ in cerebrospinal fluid (CSF) after $30 \mathrm{~min}$ of administration (24 mg/kg, i.v.) [149]. To conclude, baicalin showed great neuroprotective efficacy and BBB permeability in experimental ischemic stroke, with great potential for clinical application [150].

Nobiletin, a polymethoxylated flavone, is mainly isolated from the peel of Citrus fruits. Nobiletin was found to reduce cerebral infarction, improve motor functional deficit, and enhance BBB integrity in MCAO models [60, 141-143].
Two major strategies for nobiletin-mediated neuroprotection were inhibition of the TLR4/NF- $\kappa \mathrm{B}$ pathway to reduce inflammation and upregulation of $\mathrm{Nrf} 2 / \mathrm{HO}-1$-mediated antioxidation $[60,141,142]$. In addition, nobiletin was also found to promote neuronal survival by activating cytoprotective pathways such as the BDNF/Akt/CREB pathway and the Akt/mTOR (mammalian target of rapamycin) pathway [141, 143]. Most importantly, nobiletin might be able to cross the BBB during cerebral I/R according to a study performed by Yasuda et al. They reported that nobiletin could be rapidly accumulated in the damaged region of the ischemic brain after being administrated (i.v.) with a dosage of $15 \mathrm{mg} / \mathrm{kg}$ after reperfusion onset [142].

Scutellarin (scutellarein-7-O-glucuronide) is one of the major active components of the herb Erigeron breviscapus. Its neuroprotection in ischemic stroke has been extensively studied with various animal models. A study found that scutellarin had stronger efficacy than edaravone for reducing the infarct volume and inflammation of pMCAO rats, implying the great potential of scutellarin in clinical application [144]. Recently, the Dengzhanxixin injection (approval number Z53021569), which uses scutellarin as one of the major components, was applied to clinical ischemic stroke treatment in China. The most studied neuroprotective mechanism of scutellarin was the suppression of microglial activation and inflammation [144, 147, 151]. Besides, scutellarin also promoted microglial-mediated astrogliosis and enhanced the expression of neurotrophins in astrocytes, implying an interglial regulation mechanism for scutellarin $[67,146]$. Furthermore, scutellarin was found to improve cerebral blood flow in the ischemic brain [147]. To conclude, scutellarin is a key Chinese herbal medicine ingredient that has been primarily applied to clinical treatment, showing great potential in ischemic stroke.

3.1.2. Flavanones. Flavanones including eriodictyol [152], eriodictyol-7-O-glucoside [153], hesperidin [154], naringenin [61, 85, 155], naringin [156], neohesperidin [157], and pinocembrin $[29,158-160]$ have been reported to be neuroprotective in ischemic stroke in the past 10 years.

Naringenin naturally exists in Citrus fruits, such as grapefruit and orange. Naringenin reduced cerebral infarction and poststroke neurological deficits in both the permanent and transient MCAO rats. The neuroprotective strategies of naringenin were found to inhibit NF- $\kappa \mathrm{B}$ to lower inflammation, reduce $\mathrm{BBB}$ dysfunction, and promote Nrf2-mediated antioxidation [61, 85, 155]. Naringin (naringenin-7-O-rhamnoglucoside), a glucose derivative of naringenin, is also largely present in the Citrus species. Naringin is famous for its strong free radical scavenging activity. Feng et al. showed that naringin improved brain damage in $\mathrm{tMCAO} / \mathrm{R}$ rats by inhibiting $\mathrm{ONOO}^{-}$(peroxynitrite) and its induced excessive mitophagy [156]. Yet, naringin might not have good BBB permeability. It was found that the concentration of naringin in CSF only reached the peak of $0.95 \mu \mathrm{g} / \mathrm{mL}$ after it was administrated for $15 \mathrm{~min}(120 \mathrm{mg} / \mathrm{kg}$, i.v.) [156].

Pinocembrin exists in propolis, honey, ginger roots, and wild marjoram. It has drawn much attention in ischemic stroke treatment in the past decade. Pinocembrin exerted 
TABLE 1: Neuroprotective flavonoids and their functional mechanisms and targets ${ }^{\mathrm{a}}$.

\begin{tabular}{|c|c|c|}
\hline Compounds & Mechanisms and targets & Ref. \\
\hline \multicolumn{3}{|l|}{ Flavones (15) } \\
\hline Apigenin & $\begin{array}{c}\text { Anti-inflammation: iNOS } \downarrow, \text { COX- } 2 \downarrow \text {, p-p38 } \downarrow \text {, p-JNK } \downarrow \text {; histone deacetylases } \downarrow ; \\
\text { BDNF/CREB/Syn- } 1 \uparrow\end{array}$ & {$[77,125]$} \\
\hline APG & Antioxidation: $\mathrm{p}-\mathrm{STAT} 3 \uparrow$ & {$[126]$} \\
\hline Vitexin & Antiapoptosis: p-Erk $\uparrow, \mathrm{p}-\mathrm{JNK} \downarrow, \mathrm{p}-\mathrm{p} 38 \downarrow$ & {$[127]$} \\
\hline Baicalein & $\begin{array}{l}\text { Anti-inflammation: NF- } \kappa \mathrm{B} \downarrow \text {, p-MAPKs } \downarrow \text {, arachidonic acid release } \downarrow: 12 / 15 \text { - } \\
\text { LOX/p38 MAPK/cPLA } 2 \downarrow \text {; antiapoptosis; antiautophagy: PI3K/Akt/mTOR } \uparrow\end{array}$ & {$[82,109]$} \\
\hline Baicalin & $\begin{array}{l}\text { Antiapoptosis: p-CaMKII } \downarrow \text {; antioxidation: peroxynitrite scavenging } \uparrow \text {; anti- } \\
\text { inflammation: TLR } 2 / 4 / \mathrm{NF}-\kappa \mathrm{B} \downarrow \text {; mitochondrial function } \uparrow: \text { Drp- } 1 \downarrow, \mathrm{Mfn} 2 \downarrow \text {, } \\
\qquad \mathrm{AMPK} \alpha 1 \uparrow\end{array}$ & {$[94,128-131]$} \\
\hline Chrysin & Anti-inflammation: NF- $\kappa \mathrm{B} \downarrow, \mathrm{COX}-2 \downarrow$, iNOS $\downarrow$; antioxidation & {$[78,132,133]$} \\
\hline Diosmin & Bcl-2/Bax $\uparrow ; \mathrm{JAK} 2 / \mathrm{STAT} 3 \uparrow$ & [134] \\
\hline Ginkgetin & Antioxidation; anti-inflammation: JAK2/STAT3/SIRT1 $\downarrow$ & [135] \\
\hline Hispidulin & NLRP3-mediated pyroptosis $\downarrow ;$ AMPK/GSK3 $\beta \uparrow$ & {$[136]$} \\
\hline Luteolin & Anti-inflammation: TLR4/5/p38/NF- $\kappa \mathrm{B} \downarrow$; antioxidation; antiapoptosis & {$[95,137,138]$} \\
\hline Luteoloside & Anti-inflammation: PPAR $\gamma \uparrow / \mathrm{Nrf} 2 \uparrow / \mathrm{NF}-\kappa \mathrm{B} \downarrow$ & [139] \\
\hline Orientin & Antioxidation; anti-inflammation: TLR4/NF- $\kappa$ B/TNF- $\alpha \downarrow$; AQP- $4 \downarrow$ & {$[140]$} \\
\hline Nobiletin & $\begin{array}{c}\text { Anti-inflammation: TLR4/NF- } \kappa \mathrm{B} \downarrow \text {; antioxidation: Nrf2/HO- } 1 \uparrow \text {; antiapoptosis: } \\
\text { Akt/mTOR } \uparrow \text {; BDNF-Akt/CREB } \uparrow \text {; BBB permeability } \downarrow\end{array}$ & {$[60,141-143]$} \\
\hline Scutellarin & $\begin{array}{c}\text { Anti-inflammation: ACE/Ang II/AT1R } \downarrow \text {, microglial activation } \downarrow \text {, microglial- } \\
\text { mediated astrogliosis } \uparrow, \text { Notch-1/Nestin } \uparrow \text {; neurotrophin expression } \uparrow: \\
\text { BDNF/NGF/GDNF-Akt/CREB } \uparrow \text {; antioxidation }\end{array}$ & {$[67,144-147]$} \\
\hline Tricin 7-glucoside & Anti-inflammation: NF- $\kappa$ B activation $\downarrow$, HMGB1 expression $\downarrow$ & [148] \\
\hline \multicolumn{3}{|l|}{ Flavanones (7) } \\
\hline Eriodictyol & Anti-inflammation & [152] \\
\hline Eriodictyol-7-O-glucoside & Antioxidation in astrocytes: $\mathrm{Nrf} 2 / \mathrm{ARE} \uparrow$ & {$[153]$} \\
\hline Hesperidin & Antioxidation: NO pathway $\downarrow$ & {$[154]$} \\
\hline Naringenin & $\begin{array}{l}\text { BBB protection: NOD2/RIP2/NF- } \kappa \text { B/MMP-9 } \downarrow \text {; antiapoptosis; anti-inflammation: } \\
\qquad \text { NF- } \kappa \mathrm{B} \downarrow \text {; antioxidation: Nrf2 }\end{array}$ & {$[61,85,155]$} \\
\hline Naringin & $\mathrm{ONOO}^{-}$-mediated excessive mitophagy $\downarrow$ & [156] \\
\hline Neohesperidin & Antiapoptosis; antioxidation: Akt/Nrf2/HO- $1 \uparrow$ & {$[157]$} \\
\hline Pinocembrin & $\begin{array}{c}\text { Antiapoptosis; autophagy } \uparrow \text {; anti-inflammation: sEH/EETs } \downarrow \text {; neuronal loss } \downarrow \text {; } \\
\text { astrocyte proliferation } \downarrow\end{array}$ & {$[29,158-160]$} \\
\hline \multicolumn{3}{|l|}{ Flavanols (3) } \\
\hline (-)-Epicatechin (EC) & Anti-inflammation: microglial activation $\downarrow$; antioxidation: $\mathrm{Nrf} 2 / \mathrm{HO}-1 \uparrow$ & {$[68,163]$} \\
\hline $\begin{array}{l}\text { (-)-Epigallocatechin-3-Gallate } \\
\text { (EGCG) }\end{array}$ & $\begin{array}{l}\text { Calcium modulation and antiexcitotoxicity: TRPC6 } \\
\text { degradation } \downarrow / \text { MEK/Erk/CREB } \uparrow \text {, balance between the excitatory and inhibitory } \\
\text { amino acids } \uparrow \text {; antiapoptosis: PI3K/Akt/eNOS } \uparrow \text {; antioxidation: Nrf2/ARE } \uparrow \text {; anti- } \\
\text { inflammation: NF- } \kappa \text { B } \downarrow \text {; BBB protection: MMP- } 2 \downarrow \text {, MMP-9 } \downarrow \text {; ER stress } \downarrow\end{array}$ & {$[37,164-170]$} \\
\hline Procyanidin B2 & BBB protection; antioxidation: $\operatorname{Nrf} 2 \uparrow$ & {$[171]$} \\
\hline \multicolumn{3}{|l|}{ Flavonols (10) } \\
\hline Fisetin & $\begin{array}{l}\text { Anti-inflammation: macrophage infiltration } \downarrow \text {, microglial activation } \downarrow \text {, JNK/NF- } \\
\kappa \mathrm{B} \downarrow\end{array}$ & {$[69]$} \\
\hline Galangin & $\begin{array}{l}\text { Microenvironment of the neurovascular unit (NVU) } \uparrow: \mathrm{Wnt} / \beta \text {-catenin } \uparrow, \mathrm{HIF}- \\
1 \alpha / \mathrm{VEGF} \uparrow \text {; mitochondrial protection and antiapoptosis: } \mathrm{Bax} / \mathrm{Bcl}-2 \downarrow\end{array}$ & {$[101,175]$} \\
\hline Icariin & HDAC $\downarrow / C R E B \uparrow ;$ SIRT1/PGC- $1 \alpha \uparrow$; anti-inflammation: PPAR $\alpha / \gamma \uparrow$, NF- $\kappa \mathrm{B} \downarrow$ & {$[89,176,177]$} \\
\hline $\begin{array}{l}\text { Kaempferol-3-O-rutinoside } \\
\text { (KRS)/glucoside (KGS) }\end{array}$ & Anti-inflammation: STAT3 $\downarrow$, NF- $\kappa \mathrm{B} \downarrow$ & {$[178]$} \\
\hline $\begin{array}{l}\text { Kaempferide-7-O- }\left(4^{\prime \prime}-\mathrm{O}-\right. \\
\text { acetylrhamnosyl })-3-\mathrm{O}- \\
\text { rutinoside }\end{array}$ & Anti-inflammation; antioxidation; antiapoptosis & [179] \\
\hline
\end{tabular}


TABLe 1: Continued.

\begin{tabular}{|c|c|c|}
\hline Compounds & Mechanisms and targets & Ref. \\
\hline Quercetin & Energy metabolism $\uparrow$; antioxidation; PP2A subunit $\mathrm{B} \uparrow$; antiapoptosis & {$[180-182]$} \\
\hline Rutin & $\begin{array}{c}\text { Estrogen receptors } \uparrow: \text { BDNF/TrkB/Akt } \uparrow \text { and NGF/TrkA/CREB } \uparrow ; \\
\text { BBB protection: MMP-9 activity } \downarrow\end{array}$ & {$[119,183]$} \\
\hline Isoquercetin & $\begin{array}{c}\text { Antiapoptosis; anti-inflammation and antioxidation: Nrf2 } \uparrow \text {, } \\
\text { NOX4/ROS/NF- } \kappa \mathrm{B} \downarrow \text {, MAPK/TLR4/NF- } \kappa \mathrm{B} \downarrow\end{array}$ & {$[54,184]$} \\
\hline Isorhamnetin & $\mathrm{Nrf} 2 / \mathrm{HO}-1 \uparrow ;$ iNOS/NO $\downarrow$ & {$[185]$} \\
\hline Myricetin & Anti-inflammation: p38/NF- $\kappa \mathrm{B} \downarrow$; antioxidation; p-Akt $\uparrow$ & {$[186]$} \\
\hline \multicolumn{3}{|l|}{ Isoflavones (6) } \\
\hline Calycosin & $\begin{array}{l}\text { Anti-inflammation: microglial activation } \downarrow \text {; antiapoptosis; } \\
\text { antiautophagy; BDNF/TrkB } \uparrow \text {; calcium modulation: TRPC6/CREB }\end{array}$ & {$[43,70,190,191]$} \\
\hline Calycosin-7-O- $\beta$-D-glucoside & BBB protection: $\mathrm{NO} \downarrow / \mathrm{Cav}-1 \uparrow / \mathrm{MMPs} \downarrow$ & [192] \\
\hline Formononetin & Bax/Bcl-2 $\downarrow ;$ PI3K/Akt $\uparrow$ & {$[193]$} \\
\hline Genistein & $\begin{array}{c}\text { Antioxidation: } \mathrm{Nrf} 2 \uparrow \text {; antiapoptosis: } \mathrm{PI} 3 \mathrm{~K} / \mathrm{Akt} / \mathrm{mTOR} \uparrow \text {; Erk activation } \uparrow \text {; } \\
\text { ROS/NF- } \kappa \mathrm{B} \downarrow \text {; antiplatelet aggregation; vascular protection }\end{array}$ & [194-199] \\
\hline Daidzein & ROS production $\downarrow$ & [200] \\
\hline Puerarin & 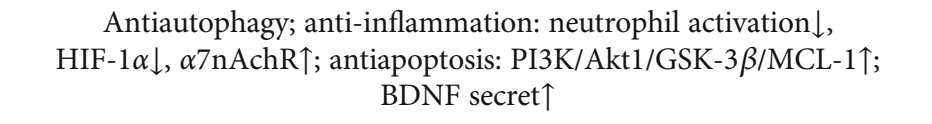 & {$[70,109,110]$} \\
\hline \multicolumn{3}{|c|}{ Anthocyanidins, chalcones, and flavonolignans (5) } \\
\hline Cyanidin-3-O-glucoside & Antiapoptosis: oxidative stress-induced AIF release $\downarrow$ & {$[211]$} \\
\hline Hydroxysafflor yellow A (HSYA) & $\begin{array}{c}\text { Antioxidation } \downarrow \text {; anti-inflammation: TLR4/MAPK/NF- } \kappa \mathrm{B} \downarrow \text {; } \\
\text { antiapoptosis: PI3K/Akt/GSK3 } \beta \uparrow, \text { mPTP opening } \downarrow \text {; } \\
\text { neurotrophin release } \uparrow: \mathrm{BDNF} \uparrow, \text { GFAP } \uparrow, \text { NGF } \uparrow \text {; autophagy } \uparrow \\
\text { Akt } \uparrow ; \text { mitochondrial function and biogenesis } \uparrow \text {; phenylalanine synthesis } \downarrow\end{array}$ & {$[98,106,212-215]$} \\
\hline Xanthohumol & Anti-inflammation; antiapoptosis; platelet activation $\downarrow$ & [216] \\
\hline Silibinin/silybin & $\begin{array}{l}\text { Anti-inflammation; antioxidation; antiapoptosis and antiautophagy: } \\
\text { PI3K/Akt/mTOR } \uparrow\end{array}$ & {$[104,217]$} \\
\hline Silymarin & Antioxidation; antiapoptosis & {$[218]$} \\
\hline
\end{tabular}

neuroprotection in experimental ischemic stroke via antiapoptosis, upregulation of autophagy, and anti-inflammation [158]. Besides, it was shown that pinocembrin inhibited the activity of soluble epoxide hydrolase $(\mathrm{sEH})$, an enzyme that degraded EETs (one of the AA metabolites) and lowered its induced neuronal damage [159]. Furthermore, pinocembrin was found to improve cognitive and memory impairments after it was administrated for $14 \mathrm{~d}$ in the global ischemia rat model [160]. Most importantly, in vitro studies found that pinocembrin could cross the $\mathrm{BBB}$ via a $\mathrm{P}$-glycoproteinconducted passive transport process [161] and extend the therapeutic time window of tPA treatment [29]. Zhao et al. reported that its neuroprotective efficacy was stronger than that of edaravone [158]. Most importantly, pinocembrin possessed high bioavailability and BBB permeability due to its good liposolubility [162]. Recently, pure synthetic pinocembrin has been subjected to a phase II clinical trial (NCT02059785) to evaluate its activity in ischemic stroke. Hereby, pinocembrin is one of the most potential drug candidates for ischemic stroke, showing great potential for clinical application.
3.1.3. Flavanols. Three flavanols are found to exert neuroprotection in ischemic stroke including (-)-epicatechin (EC) [68, 163], (-)-epigallocatechin-3-gallate (EGCG) [37, 164170], and procyanidin B2 [171].

EGCG is the most abundant catenin in green tea. EGCG has been reported to be effective in improving various CNS disorders including ischemic stroke, Alzheimer's disease, and Huntington's disease in animal models. It has been subjected to clinical trials to evaluate its efficacy in Alzheimer's disease (NCT00951834) and Huntington's disease (NCT01357681). For ischemic stroke, it was found that EGCG reduced cerebral infarction and promoted poststroke recovery in MCAO models. The neuroprotective strategies of EGCG involved the promotion of Nrf2mediated antioxidation [166], suppression of inflammation via inhibiting microglial activation and $\mathrm{NF}-\kappa \mathrm{B}[167,172]$, antiapoptosis by activating the PI3K/Akt pathway [168], and decrease of ER stress via upregulation of TRPC6 [169]. Besides, EGCG also inhibited the basal lamina degradation of the BBB by lowering the activity of MMP-9 in tMCAO/R mice [170]. Clinical studies further indicated 


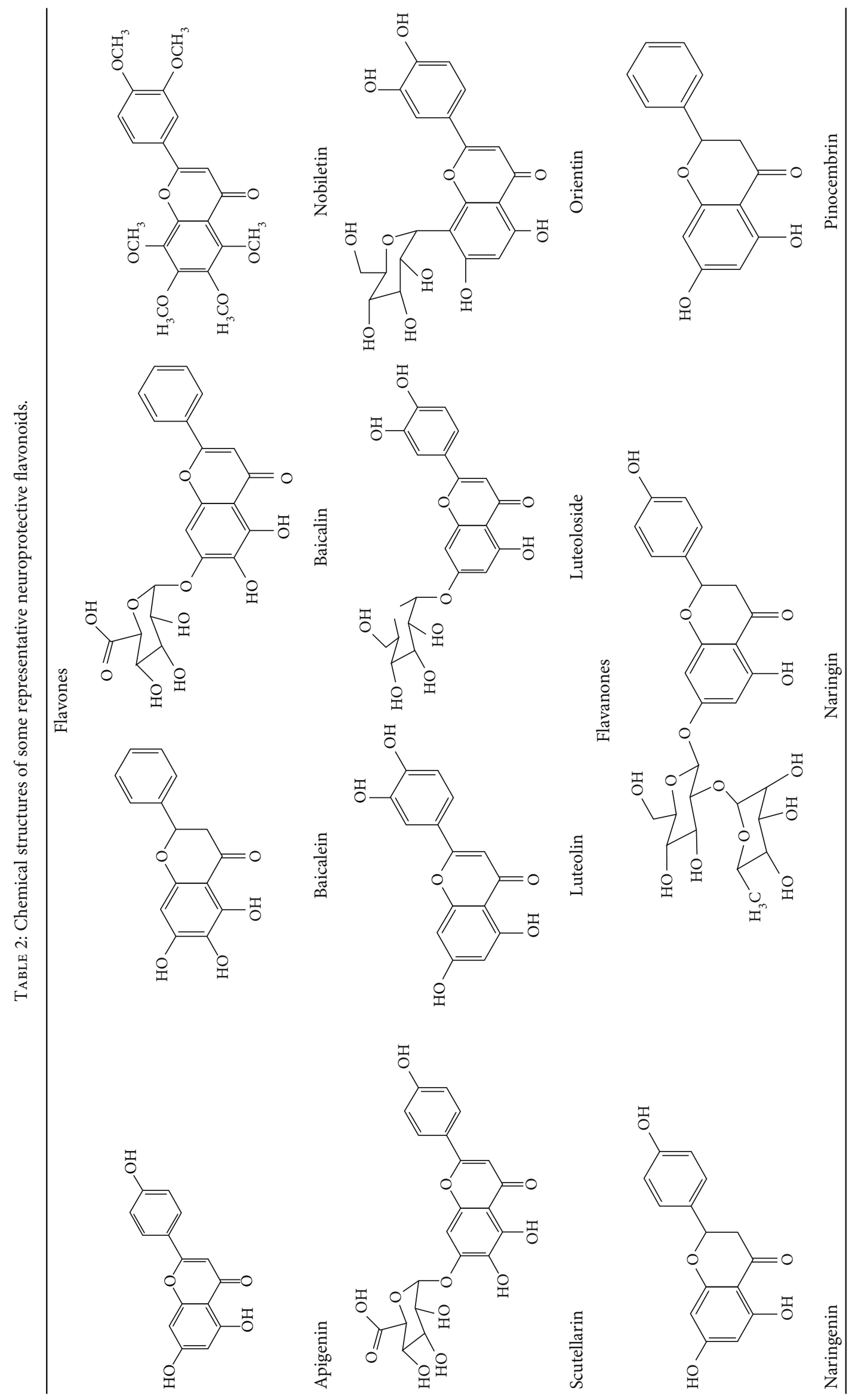




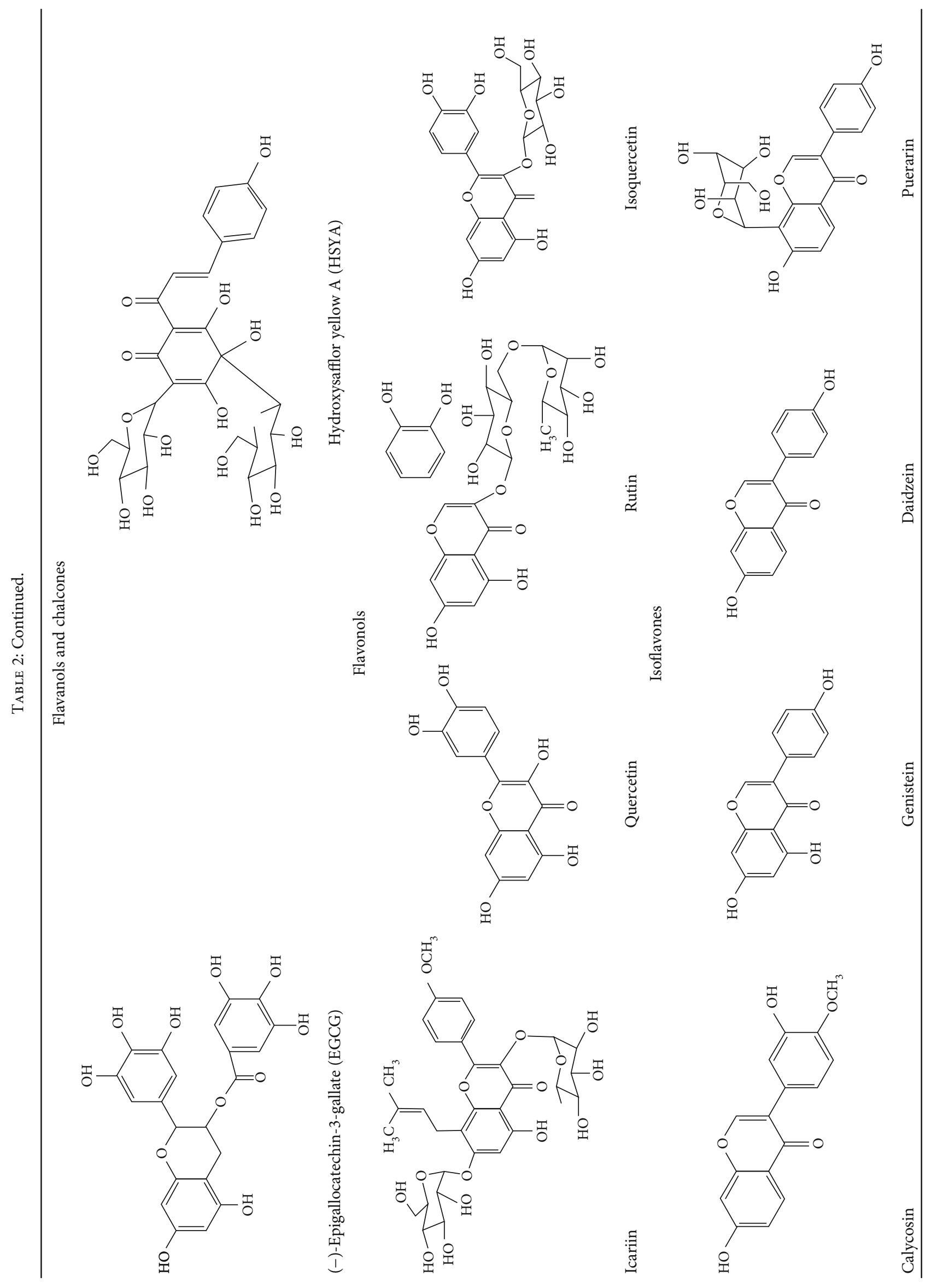


that EGCG decreased the level of MMP-2/9 in the plasma of ischemic stroke patients [164]. Furthermore, EGCG promoted long-term learning and memory recovery by maintaining the balance between the excitatory and inhibitory amino acids [37] and enhancing angiogenesis or neurogenesis $[9,173]$. Hereby, EGCG showed neuroprotective activity via regulation of multiple targets in experimental ischemic stroke. Clinically, EGCG was found to extend the therapeutic time window of tPA [165]. Previously, EGCG was considered to possess low bioavailability and BBB permeability, which disqualify it as a drug candidate for ischemic stroke treatment. However, Wei et al. recently reported that BBB permeability was greatly enhanced in aging rats, so EGCG might have great treatment potential in aged patients [174].

3.1.4. Flavonols. Neuroprotective flavonols include fisetin [69], galangin [101, 175], icariin [89, 176, 177], kaempferol-3-O-rutinoside and kaempferol-3-O-glucoside [178], kaempferide-7-O-(4" -O-acetylrhamnosyl)-3-O-rutinoside (A-F-B) [179], quercetin [180-182], rutin [119, 183], isoquercetin [54, 184], isorhamnetin [185], and myricetin [186].

Quercetin is widely distributed in fruits, vegetables, and grains, especially in apples and onions. Quercetin is often used as a dietary supplement due to its good antioxidant and anti-inflammatory activities. In experimental ischemic stroke studies, quercetin was found to reduce the brain injuries of pMCAO rats via multiple mechanisms, including antiapoptosis, promotion of autophagy, regulation of energy metabolism, and upregulation of PP2A (protein phosphatase 2) $[180-182,187]$. Recently, quercetin was subjected to a clinical trial to evaluate its effect on improving the cerebral blood flow in the aged population (NCT01376011). However, it was found that quercetin possessed low oral bioavailability $(<2 \%)$ and weak $\mathrm{BBB}$ permeability, limiting its pharmacological application in ischemic stroke [188]. Rutin (quercetin-3-rhamnosyl glucoside), a glycoside of quercetin, abundantly exists in buckwheat, passionflower, and apple. It was found that rutin $(10 \mathrm{mg} / \mathrm{kg})$ exerted similar neuroprotective efficacy as quercetin $(50 \mathrm{mg} / \mathrm{kg})$ in $\mathrm{MCAO} / \mathrm{R}$ rats, with much lower effective doses [189]. However, a direct comparison of the efficacy of quercetin and rutin should be carried out. Rutin also inhibited the activity of MMP9 and exerted BBB protective activity in a photothrombotic ischemic stroke model [183]. Furthermore, Liu et al. reported that rutin was a positive modulator of estrogen receptors. It enhanced the expression of estrogen receptors to upregulate the neurotrophin-mediated prosurvival pathways, such as the NGF/TrkA/CREB pathway and the $\mathrm{BDNF} /$ TrkB/Akt pathway in ovariectomized tMCAO/R rats [119]. Isoquercetin (quercetin-3-O-glucoside), another glycoside of quercetin, also possessed neuroprotective activity. The neuroprotective strategies of isoquercetin were found to be antioxidation mediated by Nrf2, inhibition of neuroinflammation via downregulating the TLR4/NF- $\kappa \mathrm{B}$ pathway, and antiapoptosis $[54,184]$.

3.1.5. Isoflavones. In total, 6 isoflavones are found to possess neuroprotective activity in ischemic stroke, that is, calycosin
[43, 70, 190, 191], calycosin-7-O- $\beta$-D-glucoside [192], formononetin [193], genistein [194-199], daidzein [200], and puerarin [103, 110, 201-203].

Genistein and daidzein, two major isoflavones in soybean and soy products, are described as phytoestrogens due to their structural similarity to human estrogen. Phytoestrogens can bind to the estrogen receptors and mimic the gene transcription of estrogen. However, few studies have reported the direct reaction of genistein or daidzein with estrogen receptors in ischemic stroke [204]. Instead, most of the studies focused on their antioxidant and antiapoptotic activities. It was reported that genistein or daidzein could improve neurological outcomes and reduce cerebral infarction regardless of whether it was administrated before or after MCAO via antioxidation and antiapoptosis [194, 195, 200, 205]. Besides, genistein also inhibited platelet aggregation and kept vascular reactivity in the MCAO rats, which might help to prevent clot formation [196]. Notably, genistein and daidzein played unique roles in treating postmenopausal cerebral ischemia, with several studies reporting the neuroprotective activity of genistein and daidzein in ovariectomized MCAO models. It was found that genistein pretreatment markedly decreased the neurological deficits and infarct volumes of ovariectomized MCAO rodents. The mechanisms involved the promotion of Nrf2-mediated antioxidation and inhibition of apoptosis by activating the PI3K/Akt/mTOR pathway [197199]. Besides, equol, a metabolite of daidzein, was found to exert neuroprotection in the ovariectomized $\mathrm{MCAO} / \mathrm{R}$ rats by enhancing the antioxidant defense [206]. Moreover, both genistein and daidzein could cross BBB to some extent. The efficiency of genistein was found to be below $10 \%$, while the penetration index $\left(\mathrm{AUC}_{\mathrm{CSF}} / \mathrm{AUC}_{\text {plasma }}\right)$ of daidzein was about $11.96 \%$ in SD rats $[207,208]$.

Puerarin (daidzein-8-C-glucoside) is the major bioactive component in Radix Puerariae (kudzu root). Puerarin showed marked neuroprotection in experimental ischemic stroke. It was reported that puerarin reduced the brain injuries of $\mathrm{tMCAO} / \mathrm{R}$ rats by suppression of autophagy, apoptosis, and inflammation [110, 201]. The anti-inflammatory activity of puerarin was achieved via upregulation of the cholinergic anti-inflammatory pathway, that is, promotion of $\alpha 7 \mathrm{nAchR}$ (alpha7 nicotinic acetylcholine receptor) to inhibit the JAK2/NF- $\kappa \mathrm{B}$ pathway [202]. In addition, puerarin enhanced the BDNF/PI3K/Akt pathway to promote neuronal survival and poststroke recovery $[103,203]$. Besides, the $\mathrm{AUC}_{\mathrm{CSF}} / \mathrm{AUC}_{\text {plasma }}$ of puerarin in rats was found to be about $9.29 \%$, implying its BBB permeability [208]. Notably, puerarin had shown the primary neuroprotective effect against ischemic stroke in clinical trials. For example, puerarin injection was subjected to a clinical trial, in which ischemic stroke patients were treated with conventional therapies plus an additional puerarin injection $(400 \mathrm{mg} / \mathrm{d})$ for one month. Results showed that puerarin injection significantly improved blood viscosity, neurological damage, and language function of ischemic stroke patients [209]. Besides, a meta-analysis of randomized controlled trials concluded that puerarin injection was effective and safe for clinical acute ischemic stroke treatment [210]. Hence, puerarin possessed great potential for clinical ischemic stroke treatment. 
TABLE 3: Neuroprotective stilbenoids and their functional mechanisms and targets ${ }^{\mathrm{b}}$.

\begin{tabular}{|c|c|c|}
\hline Compounds & Mechanisms and targets & Ref. \\
\hline \multicolumn{3}{|l|}{ Stilbenoids (7) } \\
\hline Resveratrol & $\begin{array}{c}\text { Anti-inflammation: T regulatory cells (Treg) } \uparrow \text {, intestinal flora-mediated immune cell } \\
\text { balance } \uparrow \text {; calcium modulation: TRPC6/MEK/CREB } \uparrow, \text { TRPC6/CaMKIV/CREB } \uparrow, \text { NMDA } \\
\text { receptor } \downarrow \text {; BDNF } \uparrow \text {; modulating energy metabolism and extending the cerebral ischemic } \\
\text { tolerance: glycolysis } \uparrow, \text { mitochondrial respiration efficiency } \uparrow \text {, phosphodiesterase } \downarrow \text {, } \\
\text { cAMP/AMPK/SIRT1 } \uparrow, \mathrm{UCP} 2 \downarrow \text {; antioxidation: Nrf2/HO-1; antiapoptosis; synaptic } \\
\text { transmission efficiency } \uparrow \text {; BBB protection: MMP-9/TIMP-1 balance } \uparrow \text {; regulation of } \\
\text { hypothalamus-pituitary-adrenal axis function; hedgehog signaling pathway } \uparrow \text {; estrogen } \\
\text { receptor } \uparrow \text {; cellular stress proteins } \uparrow\end{array}$ & {$[45,223-235]$} \\
\hline Polydatin & $\begin{array}{c}\text { BBB protection; sonic hedgehog pathway } \uparrow \text {; anti-inflammation: NF- } \kappa \mathrm{B} \downarrow \text {; antioxidation; } \\
\text { antiapoptosis }\end{array}$ & {$[236,237]$} \\
\hline Malibatol A & Mitochondrial dysfunction $\downarrow$; anti-inflammation: microglial M2 polarization $\uparrow, \operatorname{PPAR} \gamma \uparrow$ & {$[88,238]$} \\
\hline Oxyresveratrol & Antiapoptosis & [239] \\
\hline Mulberroside A & Anti-inflammation: MAPK/NF- $\kappa \mathrm{B} \downarrow$ & {$[240]$} \\
\hline Pterostilbene & Antioxidation; antiapoptosis & {$[241]$} \\
\hline $\begin{array}{l}2,3,5,4^{\prime} \text {-Tetrahydroxystilbene- } \\
2-\mathrm{O}-\beta \text {-D-glucoside }\end{array}$ & Angiogenesis $\uparrow$ & {$[242]$} \\
\hline
\end{tabular}

${ }^{\mathrm{b}}$ Notes: $\uparrow$ : activation or upregulation; $\downarrow$ : inhibition or downregulation. Abbreviation does not appear in the text. UCP2: uncoupling protein 2.

3.1.6. Anthocyanidins, Chalcones, and Flavonolignans. Cyanidin-3-O-glucoside (anthocyanidin) [211], hydroxysafflor yellow A (chalcone) [98, 106, 212-215], xanthohumol (chalcone) [216], silybin (flavonolignan) [104, 217], and silymarin (flavonolignan) [218] are neuroprotective in experimental ischemic stroke.

Hydroxysafflor yellow A (HSYA), a chalcone, is extracted from Carthamus tinctorius (safflower), a Chinese medicine that is widely used in treating cerebrovascular diseases [219]. Safflower yellow for injection (approval number Z20050146) uses HSYA as the major bioactive ingredient and has been approved in China for the treatment of cerebrovascular diseases including ischemic stroke. HSYA was found to improve cerebral infarction and cognitive impairment in animal models. The neuroprotective strategies of HSYA were multiple, including antioxidation, anti-inflammation, antiapoptosis, antiexcitotoxicity, autophagy modulation, and mitochondrial protection $[98,106,212-215,220]$. Lv et al. reported that HSYA targeted TLR4 to inhibit the NF- $\kappa$ B activation and subsequent cascade of inflammation [212, 214]. Besides, HSYA promoted the activation of the PI3K/Akt pathway to promote cytoprotective autophagy and inhibit apoptosis in MCAO rats $[106,213]$. As for mitochondrial protection, HSYA was found to improve mitochondrial function, reduce the $\mathrm{mPTP}$ opening, and promote mitochondrial biogenesis by inhibiting the production of phenylalanine [98, 215]. Notably, a single dose of $2 \mathrm{mg} / \mathrm{kg}$ (i.v.) HSYA was found to exert neuroprotection in $\mathrm{tMCAO} / \mathrm{R}$ mice, implying its high bioavailability [212]. In addition, He reported that HSYA was detected in the brain tissue homogenate of the ischemic hemisphere in MCAO rats, with the peak concentration at 90 min after HSYA administration (i.v.), indicating the BBB permeability of HSYA [221]. Hereby, HSYA had marked neuroprotective efficacy and high bioavailability and BBB permeability, so it might be a good drug candidate for ischemic stroke.
3.1.7. Summary of Flavonoids. From the current studies, baicalein and baicalin, scutellarin, pinocembrin, puerarin, and hydroxysafflor yellow A exerted great neuroprotective efficacy and high bioactivity and BBB permeability in experimental ischemic stroke. Besides, plant extracts/concentrates containing scutellarin, puerarin, and hydroxysafflor yellow A have been primarily applied in the clinical treatment of ischemic stroke due to their great neuroprotective effects. However, only pinocembrin as a single pure compound is on a phase II clinical trial at present.

3.2. Stilbenoids. Stilbenoids refer to a class of compounds that have two aromatic rings connected by an ethene bridge. Stilbenoids possess both the cis and trans forms, and the trans form is found to be more bioactive and stable [222]. In total, 7 stilbenoids are reported in the past 10 years for their neuroprotective activity in ischemic stroke, that is, resveratrol $[45,223-235]$, polydatin (resveratrol3 - $\beta$-D-glucoside) $[236,237]$, malibatol A (a resveratrol oligomer) [88, 238], oxyresveratrol [239], mulberroside A (oxyresveratrol-3, $4^{\prime}$-diglycopyranoside) [240], pterostilbene [241], and 2,3,5,4' -tetrahydroxystilbene-2-O- $\beta$-D-glucoside [242]. Their neuroprotective mechanisms are explained in Table 3, and the chemical structures of the extensively studied stilbenoids are shown in Table 4.

Resveratrol, the most famous stilbenoid, is widely distributed in plants, such as grapes. The neuroprotective effect of resveratrol in ischemic stroke has been studied extensively, and dozens of related research articles were found. It was found that resveratrol administration, especially preconditioning, improved cerebral infarction, neurological deficits, poststroke depression, and ischemic tolerance in various animal models [223]. The neuroprotective strategies of resveratrol were multiple, including the common mechanisms such as anti-inflammation, promotion of Nrf2-mediated antioxidation, antiapoptosis, and BBB protection [224-227]. In 


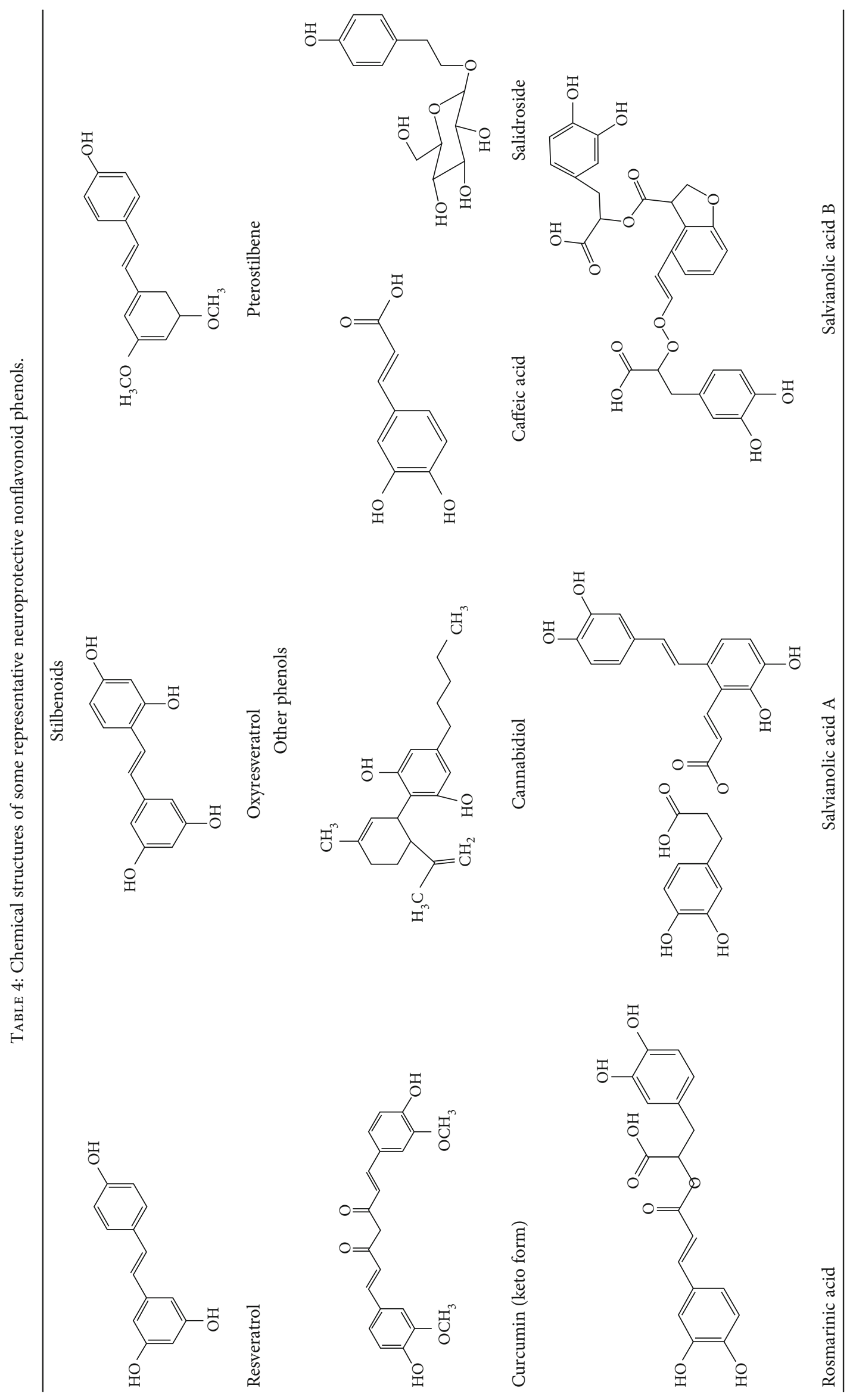


TABLE 5: Neuroprotective activity of other phenols and their mechanisms and targets ${ }^{c}$.

\begin{tabular}{|c|c|c|}
\hline Compounds & Mechanisms and targets & Ref. \\
\hline \multicolumn{3}{|l|}{ Other phenols (20) } \\
\hline Creosol & Antiexcitotoxicity; $\mathrm{Ca}^{2+}$ influx $\downarrow$ & {$[243]$} \\
\hline Curcumin & $\begin{array}{c}\text { Antiautophagy: PI3K/Akt/mTOR } \uparrow \text {; anti-inflammation: TLR4/p38/MAPK } \downarrow \text {; } \\
\text { antiapoptosis; GLUT1 and GLUT3 } \uparrow \text {; neurogenesis: Notch signaling } \\
\text { pathway } \uparrow \text {; antioxidation: Akt/Nrf2 } \uparrow \text {; mitochondrial protection: } \\
\text { SIRT1 } \uparrow ; \text { BBB protection }\end{array}$ & {$[10,96,244-249]$} \\
\hline Cannabidiol & $\begin{array}{l}\text { BBB protection; anti-inflammation; } \mathrm{Na}^{+} / \mathrm{Ca}^{2+} \text { exchangers } \uparrow ; \\
\text { antiapoptosis; antiexcitotoxicity; metabolic derangement } \downarrow\end{array}$ & {$[250-252]$} \\
\hline Hydroxytyrosol & Anti-inflammation; BDNF $\uparrow$ & {$[253]$} \\
\hline Acteoside & Antioxidation; antiapoptosis & {$[254]$} \\
\hline Hydroquinone & 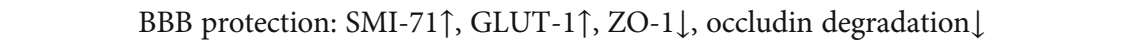 & {$[255]$} \\
\hline Lyciumamide A & Antioxidation: $\mathrm{PKC} \varepsilon / \mathrm{Nrf} 2 / \mathrm{HO}-1 \uparrow$; antiapoptosis & {$[256]$} \\
\hline Oleuropein & Antiapoptosis: Bcl-2/Bax $\uparrow$, Akt $\uparrow / G S K 3 \beta \downarrow$ & {$[257,258]$} \\
\hline Salidroside & $\begin{array}{c}\text { Anti-inflammation: microglial M2 polarization } \uparrow, \mathrm{PI} 3 \mathrm{~K} \uparrow / \mathrm{PKB} \uparrow / \mathrm{Nrf} 2 \uparrow / \mathrm{NF}-\kappa \mathrm{B} \downarrow \\
\text { PI3K/Akt/HIF } \alpha \uparrow \text {; antiapoptosis: } \mathrm{BDNF} / \mathrm{PI} 3 \mathrm{~K} / \mathrm{Akt} \uparrow ; \\
\text { complement C3 activation } \downarrow ; \text { Egrs expression } \uparrow\end{array}$ & {$[71,259-264]$} \\
\hline 6-Shogaol & Anti-inflammation: CysLT1R $\downarrow$, MAPK $\downarrow$ & [265] \\
\hline $\begin{array}{l}\text { 4-Hydroxybenzyl } \\
\text { alcohol }\end{array}$ & Antioxidation & [266] \\
\hline $\begin{array}{l}\text { 4-Methoxy benzyl } \\
\text { alcohol }\end{array}$ & BBB protection: NOS pathway $\downarrow, \mathrm{AQP}-4 \downarrow$, tight junction $\uparrow$ & [267] \\
\hline Cinnamophilin & Gray and white matter damage $\downarrow$ & {$[268]$} \\
\hline Hyperforin & TRPC6/MEK/Erk/CREB $\uparrow ;$ TRPC6/CaMKIV/CREB $\uparrow$ & [269] \\
\hline Punicalagin & Antioxidation; anti-inflammation; antiapoptosis & {$[270,271]$} \\
\hline Caffeic acid & $\begin{array}{c}\text { Antioxidation; anti-inflammation: } 5 \text {-LOX } \downarrow \text {; loss of neuronal cells } \downarrow \text {; synaptic density and } \\
\text { plasticity } \uparrow\end{array}$ & {$[80,272]$} \\
\hline Ferulic acid & Peroxiredoxin-2 $\uparrow$; thioredoxin $\uparrow$ & {$[273]$} \\
\hline Gallic acid & Antiapoptosis and mitochondrial protection: Erk $\uparrow /$ cyclophilin $\mathrm{D} \downarrow / \mathrm{mPTP} \downarrow$ & {$[99,274]$} \\
\hline Rosmarinic acid & Anti-inflammation: HMGB1/NF- $\kappa \mathrm{B} \downarrow$; synaptogenic activity $\uparrow ; \mathrm{BDNF} \uparrow ; \mathrm{BBB}$ protection & {$[275,276]$} \\
\hline Salvianolic acid A & Antioxidation; anti-inflammation; metabolic dysfunction $\downarrow$ & [277] \\
\hline
\end{tabular}

addition, resveratrol was also reported to modulate the energy metabolism of the ischemic brain. It was found that resveratrol enhanced bioenergetic efficiency such as improving glycolysis and mitochondrial respiration efficiency to extend the window of ischemic tolerance, especially in elderly individuals [228-232]. Further studies revealed that this process was achieved by activating AMPK and SIRT1, an NAD ${ }^{+}$dependent deacetylase that can induce adaptive responses under energy depletion conditions [228-232]. Furthermore, resveratrol maintained intracellular calcium homeostasis via the promotion of the TRPC6/CREB pathway and inhibition of the NMDA receptor [45] and activated sonic hedgehog signaling, a pathway that contributes to neurogenesis and neurological recovery $[233,234]$. Notably, a novel study that focused on the gut-brain axis indicated that resveratrol inhibited inflammation via modulating the intestinal floramediated immune cell balance such as the Th1/Th2 balance and Treg/Th17 balance in the lamina propria of the small intestine, proposing an original hypothesis for resveratrolmediated neuroprotection [235].
In summary, resveratrol possessed marked neuroprotective activity in experimental ischemic stroke and might have great potential in improving the ischemic tolerance when administrated before cerebral ischemia occurs.

3.3. Other Phenols. Other phenols refer to the phenolic compounds apart from flavonoids and stilbenoids, such as phenolic alcohols, phenolic acids, and lignans. In total, 20 other types of phenols are found to possess neuroprotection in ischemic stroke, including creosol [243], curcumin [10, 96, 244-249], cannabidiol [250-252], hydroxytyrosol [253], acteoside [254], hydroquinone [255], lyciumamide A [256], oleuropein [257, 258], salidroside [71, 259-264], 6-shogaol [265], 4-hydroxybenzyl alcohol [266], 4-methoxy benzyl alcohol [267], cinnamophilin [268], hyperforin [269], punicalagin [270, 271], caffeic acid [80, 272], ferulic acid [273], gallic acid $[99,274]$, rosmarinic acid $[275,276]$, and salvianolic acid A [277]. Their neuroprotective mechanisms are clarified in Table 5, and the chemical structures of the extensively studied ones are shown in Table 4. 
Curcumin is extracted from the root of Curcuma longa (turmeric), a common spice plant in Asian countries [278]. It was found that curcumin reduced cerebral infarction, neurological deficits, and brain edema in tMCAO models regarding preconditioning or postconditioning. Curcumin is a pleiotropic agent for neuroprotection, modulating multiple mechanisms including antiapoptosis, inhibition of inflammation by downregulating TLR4, promotion of Akt/Nrf2-mediated antioxidation, suppression of autophagy by activating the PI3K/Akt/mTOR pathway, protection of mitochondria via upregulating SIRT1, the decrease of ER stress, and BBB protection [96, 244-248, 279]. In addition, curcumin promoted neurogenesis via activation of the Notch signaling pathway that improves poststroke recovery [10]. Xia et al. reported that curcumin also showed neuroprotection in a diabetic stroke model. The mechanism involved antiapoptosis and promotion of glucose uptake by activating GLUT1/3 (glucose transporter 1/3) [249]. Curcumin is one of the most popular phytochemicals in pharmacological research, with numerous clinical trials conducted for various clinical disorders including CNS diseases. As an example, curcumin has been subjected to several clinical trials for Alzheimer's disease (NCT00164749, NCT00099710, and NCT01001637). Yet, those clinical trials failed due to limited bioavailability and the BBB permeability of curcumin, limiting its clinical application in ischemic stroke. Recently, researchers were trying to enhance the bioavailability of curcumin via modulation of its chemical structure or use of the solid lipid particle method. To illustrate, Wicha et al. found that hexahydrocurcumin exerted neuroprotection with lower doses, showing better bioavailability than curcumin [280].

Cannabidiol (CBD), a phytocannabinoid from Cannabis sativa, is found to be nonpsychoactive and possesses cytoprotective activities. Cannabidiol has been studied in various therapeutic uses, especially for CNS disorders [281]. For ischemic stroke, cannabidiol was reported to exert neuroprotection with relatively low effective doses (single dose of $5 \mathrm{mg} / \mathrm{kg}$, i.p.) in MCAO models [250-252]. The neuroprotective strategies of cannabidiol included antiapoptosis, antiexcitotoxicity, anti-inflammation, $\mathrm{BBB}$ protection, $\mathrm{Ca}^{2+}$ modulation, and metabolism regulation [250-252]. In addition, a meta-analysis that reviewed 34 publications of cannabidiol-mediated ischemic stroke indicated that cannabidiol markedly reduced cerebral I/R-induced infarction [282]. Most importantly, cannabidiol was highly lipophilic and could easily cross $\mathrm{BBB}$, reaching a relatively high concentration quickly after administration [283]. Notably, more than 100 clinical trials related to the therapeutic application of cannabidiol are being conducted or are completed at present, with several trials for CNS disorders. For example, two phase II clinical trials are being conducted to evaluate the efficacy of cannabidiol on motor and tremor symptom improvement in Parkinson's disease (NCT03582137, NCT02818777).

Salidroside is the main bioactive component of Rhodiola rosea L. [284] and showed neuroprotection for ischemic stroke in various MCAO models. The major neuroprotective strategies of salidroside involved anti-inflammation and antiapoptosis. Liu et al. found that salidroside suppressed inflam- mation by promoting microglial M2 polarization [71]. Other studies showed that salidroside inhibited NF- $\kappa \mathrm{B}$ and activated HIF $\alpha$ (hypoxia-inducible factors) via upregulation of the PI3K/Akt pathway, providing another mechanism for its anti-inflammatory activity [261, 263, 264]. In addition, Zhang et al. reported that the antiapoptotic effect of salidroside was achieved via activation of the BDNF/PI3K/Akt pathway in tMCAO/R mice [262]. Furthermore, salidroside upregulated the cytoprotective transcriptional factor Egrs (early growth response genes) to improve neuronal activity and synaptic plasticity $[259,260]$. However, the concentration of salidroside in the brain is extremely low after administration $(15 \mathrm{mg} / \mathrm{kg}$, i.v.), indicating that salidroside might have difficulty crossing BBB [285].

Salvianolic acids are the bioactive compounds extracted from the roots of Salvia metrorrhagia (Danshen), a traditional Chinese medicine for treating cardiovascular disease [286]. Salvianolic acid A was reported to improve brain damage in $\mathrm{MCAO} / \mathrm{R}$ rats by its antioxidant, anti-inflammatory, and metabolism regulatory activities [277]. Salvianolic acids for injection (SAFI), a commercially available Chinese herb medicine developed by Tianjin Tably Pride Pharmaceutical Company, has been approved for the treatment of ischemic stroke in the recovery phase in China. SAFI is composed of five natural phenolic acids: salvianolic acid B (68.31\%), salvianolic acid D (3.7\%), salvianolic acid Y (5.1\%), alkannic acid (3.86\%), and rosmarinic acid (2.68\%) [287]. In experimental ischemic stroke, SAFI was found to promote poststroke recovery through two major mechanisms: promotion of neurogenesis via activation of the sonic hedgehog pathway and via upregulation of neurotrophins such as BDNF and NGF [288]. Besides, SAFI also reduced brain damage in the acute phase of ischemic stroke. The mechanisms involved anti-inflammation via inhibition of microglial activation, as well as maintaining mitochondrial permeability in astrocytes through activation of the PI3K/Akt/mtCx43 (mitochondrial connexin 43) pathway [286, 287].

Caffeic acid is widely present in dietary plants such as fruits, vegetables, coffee, and olive oils. It was found that caffeic acid reduced cerebral infarction and improved poststroke learning, memory, and spatial deficits in global ischemia or pMCAO models $[80,272]$. The mechanisms involved suppression of oxidative stress, inhibition of 5-LOX-induced inflammation, and reduction of synaptic dysfunction by upregulation of synaptophysin, a biomarker for synaptic density and plasticity $[80,272]$. Rosmarinic acid, an ester of caffeic acid, exists in plants of the Lamiaceae family such as rosemary and perilla. Rosmarinic acid attenuated brain damage and memory deficits by promoting synaptogenic activity, suppressing inflammation, and upregulating BDNF in pMCAO mice. In addition, rosmarinic acid also exerted neuroprotection in a diabetic ischemic stroke model by promoting BBB function and inhibiting inflammation. As mentioned above, rosmarinic acid is one of the components of SAFI, accounting for $2.68 \%$ of the injection.

In summary, salvianolic acids and rosmarinic acid have been primarily applied as key ingredients in a Chinese herbal medicine for clinical ischemic stroke treatment. Yet, there is no clinical data to prove their individual effectiveness at 
TABLE 6: Neuroprotective terpenoids and their functional mechanisms and targets ${ }^{\mathrm{d}}$.

\begin{tabular}{|c|c|c|}
\hline Compounds & Mechanisms and targets & Ref. \\
\hline \multicolumn{3}{|l|}{ Monoterpenoids (14) } \\
\hline Borneol & Antiapoptosis; anti-inflammation; neurovascular unit function $\uparrow$ & {$[289,290]$} \\
\hline Carvacrol & $\begin{array}{c}\text { Ferroptosis } \downarrow \text {; antioxidation; GPx4 } \uparrow \text {; TRPM7 } \downarrow \text {; antiapoptosis: Bcl-2/Bax } \uparrow \text {, } \\
\text { PI3K/Akt } \uparrow \text {; anti-inflammation: NF- } \kappa \mathrm{B} \downarrow\end{array}$ & {$[46,291-293]$} \\
\hline Catalpol & Angiogenesis $\uparrow$; JAK2/STAT3 $\uparrow$; ATPase activity $\uparrow$; excitatory amino acid toxicity $\downarrow$ & {$[294,295]$} \\
\hline Cornin & Mitochondrial protection; antioxidation & [296] \\
\hline Genipin & Antiapoptosis; UCP2/SIRT3 $\downarrow$ & [297] \\
\hline Geniposide & Antiapoptosis; BBB protection; GluN2A/Akt/Erk $\uparrow$ & {$[41]$} \\
\hline Linalool & Phospholipid homeostasis $\uparrow$ & [298] \\
\hline$\beta$-Myrcene & Antioxidation: free radical scavenging & [299] \\
\hline Paeoniflorin & $\begin{array}{c}\text { Calcium modulation: } \mathrm{Ca}^{2+} \downarrow / \mathrm{CaMKII} \uparrow / \mathrm{CREB} \uparrow \text {; anti-inflammation: } \\
\text { MAPK/NF- } \kappa \mathrm{B} \downarrow \text {; antiapoptosis }\end{array}$ & [300-302] \\
\hline Perillaldehyde & Anti-inflammation: JNK $\downarrow$; antiapoptosis: Akt $\uparrow$ & {$[303]$} \\
\hline Perillyl alcohol & Anti-inflammation; antioxidation & {$[304]$} \\
\hline$\alpha$-Pinene & Antioxidation; anti-inflammation & {$[305]$} \\
\hline Picroside II & $\begin{array}{c}\text { Antioxidation: Rac-1/NOX } 2 \downarrow \text {; antiapoptosis: mPTP permeability } \downarrow \text {; } \\
\text { anti-inflammation: MEK/Erk1/2/COX-2 } \downarrow \text {; BBB protection: } \\
\text { ROCK/MLCK/MMP- } 2 \downarrow / \text { claudin- } 5 \uparrow\end{array}$ & $\begin{array}{l}{[56,79,100} \\
306,307]\end{array}$ \\
\hline Safranal & Antioxidation & [308] \\
\hline \multicolumn{3}{|l|}{ Sesquiterpenoids (8) } \\
\hline Alantolactone & Anti-inflammation: MAPK/NF- $\kappa \mathrm{B} \downarrow$ & [312] \\
\hline Atractylenolide III & Anti-inflammation: mitochondrial fission in microglia $\downarrow$, JAK2/STAT3/Drp-1 $\downarrow$ & {$[86]$} \\
\hline Bakkenolide IIIa & Antioxidation; anti-inflammation: Erk $\downarrow$, Akt/NF- $\kappa \mathrm{B} \downarrow$ & [313] \\
\hline Bilobalide & $\begin{array}{l}\text { Mitochondrial protection: complex I function } \uparrow \text {; antiexcitotoxicity; anti-inflammation: } \\
\text { JNK1/2 } \downarrow \text {, p38 MAPK } \downarrow \text {; antiautophagy; antiapoptosis; angiogenesis } \uparrow: \text { Akt/eNOS } \uparrow\end{array}$ & {$[314-317]$} \\
\hline$(-)-\alpha$-Bisabolol & Anti-inflammation & [318] \\
\hline Parthenolide & BBB permeability $\downarrow$; caspase- $1 / \mathrm{p} 38 / \mathrm{NF}-\kappa \mathrm{B} \downarrow$ & {$[319]$} \\
\hline Patchouli alcohol & Anti-inflammation & {$[320]$} \\
\hline$\beta$-Caryophyllene & Anti-inflammation: microglial M2 polarization $\uparrow, T L R 4 \downarrow$ & {$[321]$} \\
\hline \multicolumn{3}{|l|}{ Diterpenoids (11) } \\
\hline Andrographolide & $\begin{array}{l}\text { Anti-inflammation: microglial activation } \downarrow \text {, PI3K/Akt-NF- } \kappa \text { B/HIF- } 1 \alpha \downarrow \text {, } \\
\text { astrocyte activation } \downarrow \text {, iNOS; BBB permeability } \downarrow \text {; antioxidation: } \\
\text { p38/Nrf2/HO- } 1 \uparrow, \text { gp9 } 1^{\text {phox }} / \mathrm{NOX} 2 \downarrow \text {; BDNF/TrkB } \uparrow\end{array}$ & {$[57,325-328]$} \\
\hline Erinacine A & Anti-inflammation: iNOS, p38, and CHOP $\downarrow$ & [329] \\
\hline Ginkgolide B & $\begin{array}{l}\text { Anti-inflammation: microglial M2 polarization } \uparrow, \mathrm{NF}-\kappa \mathrm{B} \downarrow \\
\text { PAF receptor } \downarrow \text {; antiapoptosis; antiexcitotoxicity: imbalance of } \\
\text { excitatory and inhibitory amino acids } \downarrow ; \mathrm{BBB} \text { permeability } \downarrow\end{array}$ & $\begin{array}{l}{[38,72,330} \\
331]\end{array}$ \\
\hline Ginkgolide K & Antioxidation; neurogenesis: JAK2/STAT3个 & {$[11,332]$} \\
\hline Pseudopterosin A & Antioxidation; anti-inflammation; antiapoptosis: Akt $\uparrow$ & [333] \\
\hline Salvinorin A & Mitochondrial function $\uparrow:$ AMPK/Mfn2 $\uparrow$, kappa opioid receptor $\uparrow$ & [334] \\
\hline Tanshinone I & Neuronal death $\downarrow$; anti-inflammation & [335] \\
\hline Tanshinone IIA & $\begin{array}{c}\text { Antiapoptosis: PI3K/Akt } \uparrow \text {; anti-inflammation: } \mathrm{HMGB} 1 / \mathrm{NF}-\kappa \mathrm{B} \downarrow \\
\text { MIF/NF- } \kappa \mathrm{B} \downarrow \text {, astrocyte activation, MAPKs } \downarrow \text {, PPAR } \gamma \uparrow ; \\
\text { antioxidation; TORC } 1 \uparrow ; \text { BDNF/CREB } \uparrow\end{array}$ & {$[75,336-343]$} \\
\hline Totarol & Antioxidation: Akt/HO- $1 \uparrow$ & {$[344]$} \\
\hline Triptolide & BBB permeability $\downarrow$; anti-inflammation: $\mathrm{p} 38 / \mathrm{NF}-\kappa \mathrm{B} \downarrow$; autophagy $\uparrow$; antiapoptosis & $\begin{array}{l}{[113,345,} \\
346]\end{array}$ \\
\hline $\begin{array}{l}(1 \mathrm{~S}, 2 \mathrm{E}, 4 \mathrm{R}, 6 \mathrm{R},-7 \mathrm{E}, 11 \mathrm{E})-2,7,11- \\
\text { Cembratriene-4,6-diol }\end{array}$ & Antiapoptosis: PI3K/Akt $\uparrow$; ICAM-1 $\downarrow$ & {$[347]$} \\
\hline
\end{tabular}


TABLE 6: Continued.

\begin{tabular}{|c|c|c|}
\hline Compounds & Mechanisms and targets & Ref. \\
\hline \multicolumn{3}{|l|}{ Triterpenoids (20) } \\
\hline Arjunolic acid & Antioxidation & [357] \\
\hline Asiatic acid & Antiapoptosis and mitochondrial protection: cytochrome $\mathrm{c}$ and AIF release $\downarrow$; MMP-9 $\downarrow$ & {$[358,359]$} \\
\hline Acetyl-11-keto- $\beta$-boswellic acid & Antioxidation: Nrf2/HO-1个; anti-inflammation: 5-LOX, NF- $\kappa \mathrm{B} \downarrow$ & {$[81,360,361]$} \\
\hline 11-Keto- $\beta$-boswellic acid & Antioxidation: $\mathrm{Nrf} 2 / \mathrm{HO}-1 \uparrow$ & $\begin{array}{l}\text { Ding et al. } \\
\text { (2015) }\end{array}$ \\
\hline 28-O-Caffeoyl betulin & Anti-inflammation; hypothermic effects & [362] \\
\hline Celastrol & Anti-inflammation: microglial M2 polarization $\uparrow$, IL-33/ST2 $\downarrow$, JNK/c-Jun/NF- $\kappa \mathrm{B} \downarrow$ & {$[73,363]$} \\
\hline Echinocystic acid & Antiapoptosis; anti-inflammation: JNK $\downarrow$ & [364] \\
\hline $18 \beta$-Glycyrrhetinic acid & Antioxidation; antiapoptosis & [365] \\
\hline Maslinic acid & Synaptogenesis $\uparrow:$ axonal regeneration $\uparrow$, Akt/GSK-3 $\beta \uparrow$ & [366] \\
\hline Ursolic acid & Anti-inflammation; antioxidation: Nrf2 $\uparrow$ & [367] \\
\hline Madecassoside & Antioxidation; antiapoptosis; anti-inflammation & {$[368]$} \\
\hline Astragaloside IV & $\begin{array}{l}\text { Antiapoptosis: P62-LC3-autophagy } \uparrow \text {; antioxidation: Nrf2 } \uparrow \text {; } \\
\text { mitochondrial protection: Akt/hexokinase-II } \uparrow \text {; anti-inflammation }\end{array}$ & $\begin{array}{l}{[62,114,369} \\
370]\end{array}$ \\
\hline Glycyrrhizin & $\begin{array}{c}\text { Anti-inflammation: HMGB1/TLR4/IL-17A } \downarrow \text {; antioxidation; } \\
\text { antiexcitotoxicity; antiapoptosis }\end{array}$ & {$[91,371-373]$} \\
\hline Diammonium glycyrrhizinate & Anti-inflammation & {$[374]$} \\
\hline Ginsenoside Rb1 & 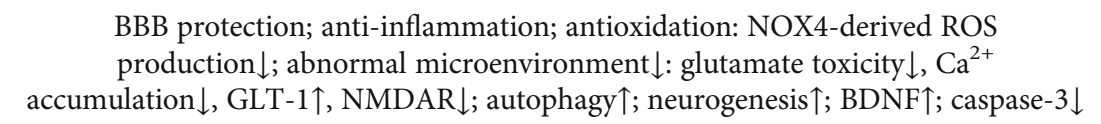 & $\begin{array}{l}{[34,55,115} \\
375,376]\end{array}$ \\
\hline Ginsenoside Rd & $\begin{array}{c}\text { Anti-inflammation: microglial proteasome-mediated NF- } \kappa \text { B activation } \downarrow \text {, PARP- } 1 \downarrow \text {; } \\
\text { antioxidation: free radical scavenging; antiapoptosis; mitochondrial protection; energy } \\
\text { restoration; } \mathrm{Ca}^{2+} \text { modulation: TRPM7 } \downarrow \text {, ASIC1 a } \downarrow \text {, ASIC2 a } \uparrow \text {; DNA damage } \downarrow \text { : } \\
\text { NEIL1/3 } \uparrow\end{array}$ & {$[49,377-383]$} \\
\hline Ginsenoside Rg1 & $\begin{array}{c}\text { Anti-inflammation: microglial proteasome-mediated NF- } \kappa \mathrm{B} \text { activation } \downarrow \text {; BDNF } \uparrow \text {; } \\
\text { excitatory amino acid } \downarrow \text {; antioxidation: miR-144 } \downarrow / \mathrm{Nrf} 2 \uparrow / \mathrm{ARE} \uparrow \text {; angiogenesis } \uparrow: \\
\text { PI3K/Akt/mTOR } \uparrow \text {; BBB permeability } \downarrow \text { : aquaporin- } 4 \downarrow \text {, PAR- } 1 \downarrow\end{array}$ & [384-389] \\
\hline 20(R)-Ginsenoside Rg3 & Antiapoptosis: calpain $I \downarrow$, caspase- $3 \downarrow$ & [390] \\
\hline Pseudoginsenoside F11 & Antiapoptosis; autophagic/lysosomal defects $\downarrow ; \mathrm{Ca}^{2+}$ overload $\downarrow$ & {$[391,392]$} \\
\hline Notoginsenoside R1 & Antiapoptosis; mitochondrial protection; estrogen receptor-Akt/Nrf $2 \uparrow$ & [393] \\
\hline \multicolumn{3}{|l|}{ Tetraterpenoids (3) } \\
\hline Astaxanthin & Antioxidation; antiapoptosis; neurogenesis $\uparrow$; neurotrophin expression: $\mathrm{BDNF} \uparrow, \mathrm{NGF} \uparrow$ & $\begin{array}{l}{[120,400,} \\
\quad 401]\end{array}$ \\
\hline Fucoxanthin & Antioxidation: Nrf2/HO-1个 & [402] \\
\hline Lutein & Antiapoptosis; antioxidation; anti-inflammation & [403] \\
\hline
\end{tabular}

${ }^{\mathrm{d}}$ Notes: $\uparrow$ : activation or upregulation; $\downarrow$ : inhibition or downregulation. Abbreviations do not appear in the text. CHOP: C/EBP homologous protein; GPx4: glutathione peroxidase 4; MLCK: myosin light chain kinase; PAR-1: protease-activated receptors; ROCK: Rho-associated kinase.

present. Cannabidiol showed good neuroprotective efficacy and high BBB permeability, so it was a good candidate for ischemic stroke drug development. Although curcumin and salidroside showed high neuroprotective efficacy, the poor bioavailability and BBB permeability might limit their further clinical applications.

3.4. Terpenoids. Terpenoids are a class of compounds that have an isoprene unit as their basic component. Depending on the number of isoprene units, terpenoids can be divided into monoterpenoids (two units), sesquiterpenoids (three units), diterpenoids (four units), triterpenoids (six units), and tetraterpenoids (eight units). In total, 56 terpenoids were found to possess neuroprotective activity for ischemic stroke after searching the recent 10 years of studies in PubMed with keywords "Terpenoids, Stroke, Neuroprotection." The neuroprotective terpenoids and their functional mechanisms are listed in Table 6, and the chemical structures of the extensively studied ones are shown in Table 7.

3.4.1. Monoterpenoids. The neuroprotective monoterpenoids include borneol [289, 290], carvacrol [46, 291-293], catalpol [294, 295], cornin [296], genipin [297], geniposide [41], linalool [298], $\beta$-myrcene [299], paeoniflorin [300-302], perillaldehyde [303], perillyl alcohol [304], $\alpha$-pinene [305], picroside II [56, 79, 100, 306, 307], and safranal [308].

Borneol is present in the plants of Artemisia and Dipterocarpaceae and has been used in traditional Chinese 


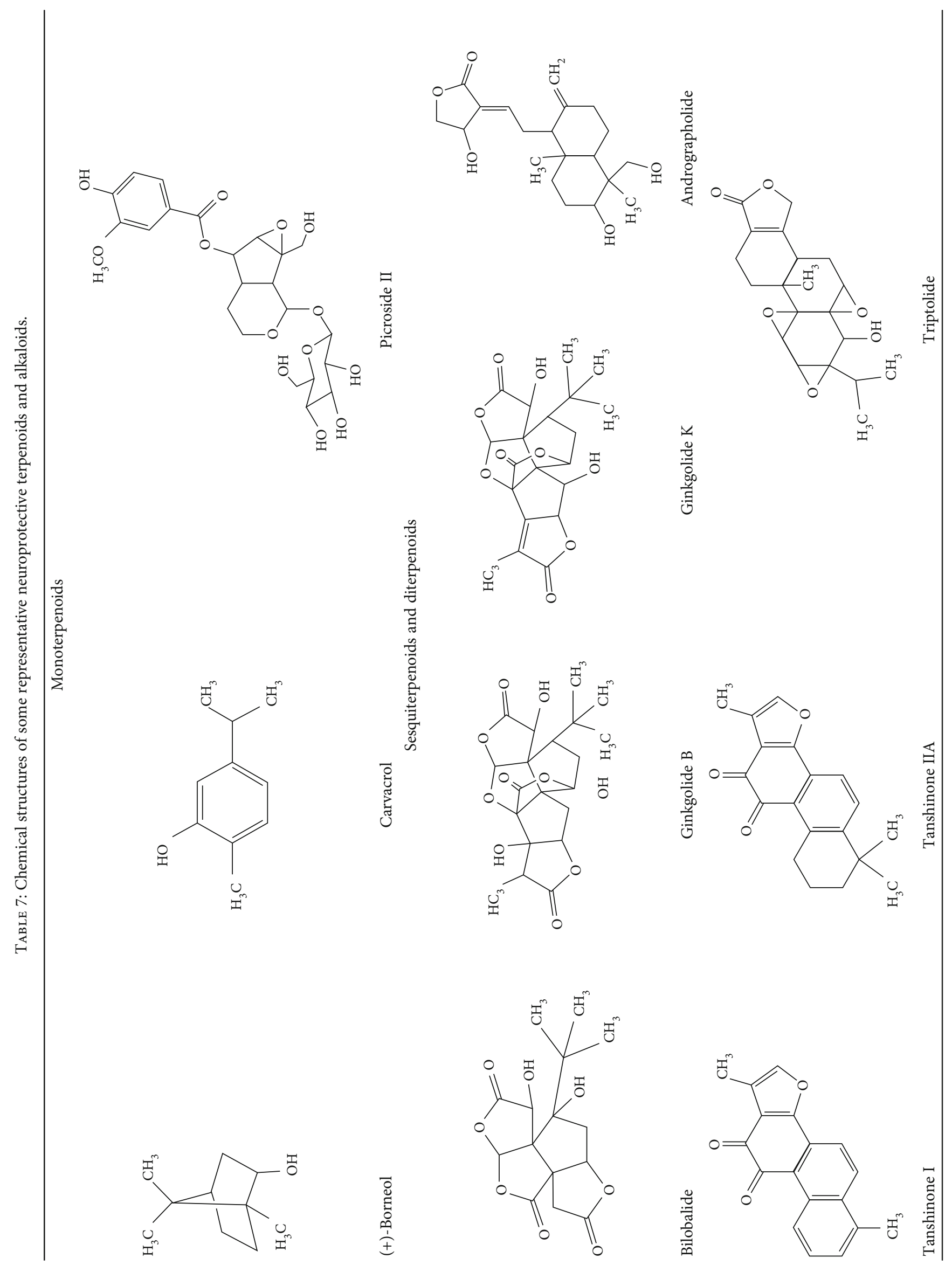




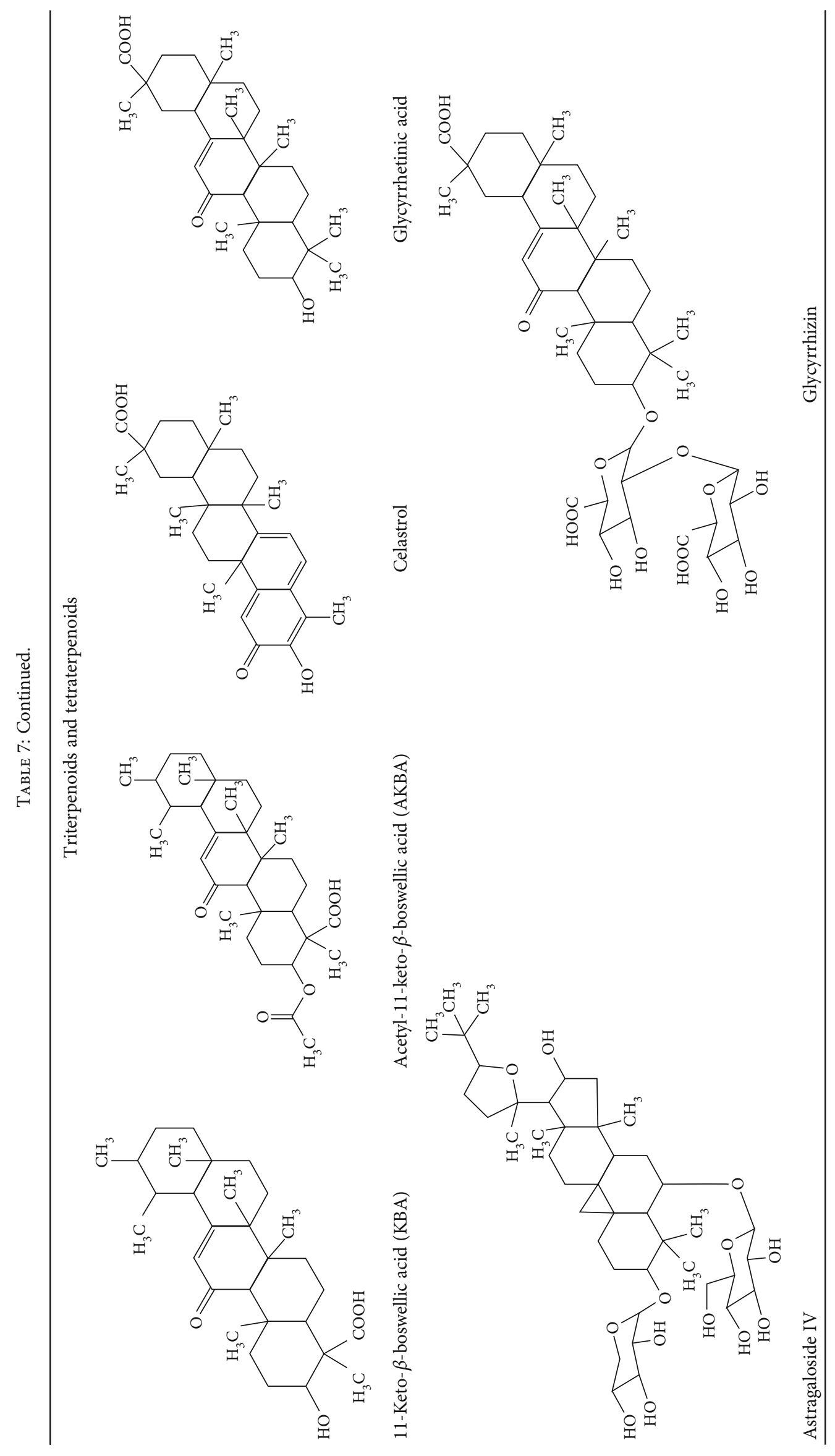




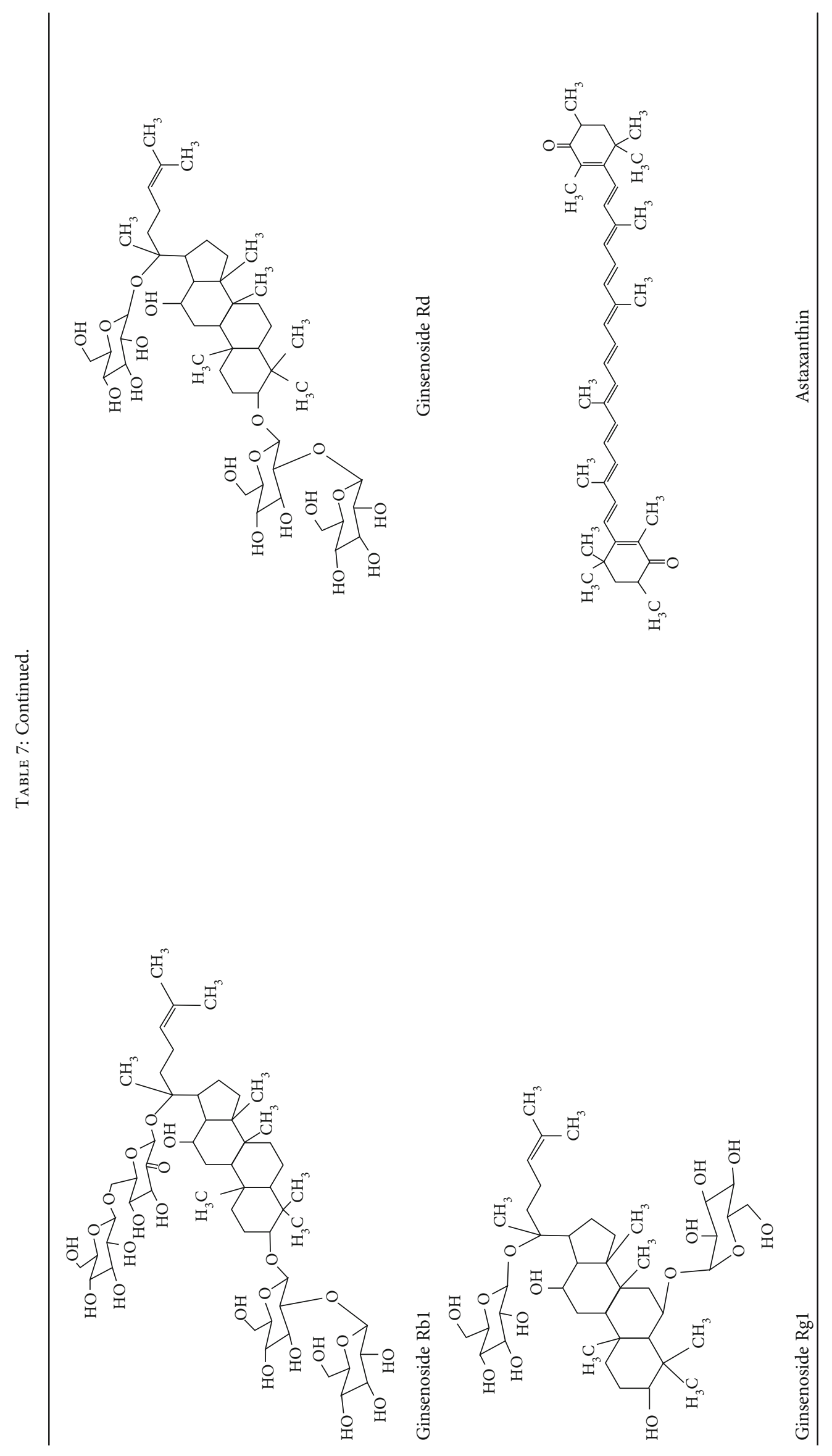




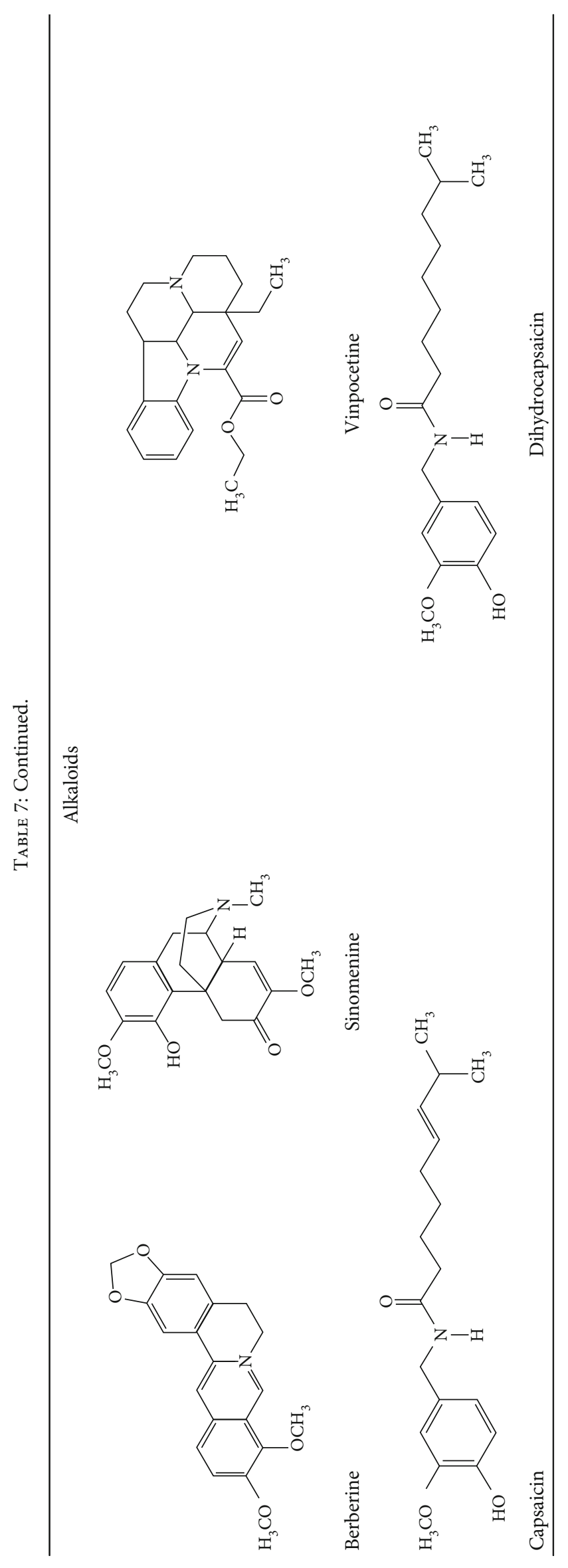


medicine to restore consciousness after stroke, coma, or other brain injuries for more than 1500 years [309]. There are three types of borneols naturally existing in the herbs: (-)-borneol, (+)-borneol, and isoborneol. Dong et al. compared the neuroprotective effect of these three borneols in pMCAO rats, finding that (-)-borneol possessed the strongest protective efficacy in reducing cerebral infarction, neurological deficits, and brain edema. The mechanism of (-)-borneol included antiapoptosis, anti-inflammation, and protection of the neurovascular units [289]. Notably, the most attractive property of borneol was its strong BBB permeability. Hereby, borneol was usually used as an "assistant" drug in traditional Chinese medicine to deliver neuroprotective medications into the brain, enhancing their therapeutic efficacy [310]. Notably, (+)-borneol possessed synergistic effects with edaravone. It was found that (+)-borneol enhanced the neuroprotective efficacy of edaravone, promoted edaravone-mediated long-term recovery, and extended its therapeutic window in the MCAO model [290]. Accordingly, a sublingual tablet consisting of edaravone and borneol was subjected to a phase I clinical trial to test its safety, tolerability, and pharmacokinetics (NCT03495206). Recently, the edaravone and dexborneol concentrated solution for injection (approval number H20200007), developed by Simcere Pharmacological Company, was approved in China in 2020. Hence, borneol has great potential to be used as an upper ushering drug in ischemic stroke.

Carvacrol is one of the major ingredients in the essential oil of oregano and thyme and is widely used as a food additive. Studies showed that it was lipophilic and able to cross BBB [311]. Carvacrol was found to reduce cerebral infarction and neurological deficits in MCAO models [46, 291-293]. Neuroprotective strategies of carvacrol involved antiinflammation via inhibition of NF- $\kappa \mathrm{B}$ and antiapoptosis via suppression of TRPM7 and promotion of the PI3K/Akt pathway $[46,292]$. Besides, carvacrol also improved poststroke learning and memory recovery in the global ischemia gerbil model via antioxidation and inhibition of ferroptosis, a programmed cell death pathway induced by iron ions and ROS [291]. Notably, carvacrol possessed an extended therapeutic window, exerting protective effects even when administrated (i.c.v.) at $6 \mathrm{~h}$ after reperfusion [293].

3.4.2. Sesquiterpenoids. In total, 8 sesquiterpenoids are found to exhibit neuroprotection in ischemic stroke, including alantolactone [312], atractylenolide III [86], bakkenolide IIIa [313], bilobalide [314-317], (-)- $\alpha$-bisabolol [318], parthenolide [319], patchouli alcohol [320], and $\beta$-caryophyllene [321].

The Ginkgo biloba leaf extract EGb761, consisting of flavonol glycosides (24\%) and terpene lactones (6\%), has been reported to exhibit neuroprotection in different CNS disorders such as stroke and Alzheimer's disease [322]. Bilobalide, one of the major bioactive terpenoids in EGb761 (accounting for $3 \%$ of EGb761), is widely studied in ischemic stroke. It was found that bilobalide showed neuroprotection in $\mathrm{tMCAO} / \mathrm{R}$ models regardless of whether it was administrated before or after ischemia [314-317]. The neuroprotective mechanisms of bilobalide are multiple. It was found that bilobalide restored the energy supply via protection of complex I in mitochondria and reduced the damage induced by energy depletion such as glutamate release, intracellular $\mathrm{Ca}^{2+}$ accumulation, and mitochondrial swelling [315, 316]. Besides, bilobalide also possessed antioxidant and anti-inflammatory activities by upregulation of JNK1/2 and p38 [314, 323]. Furthermore, bilobalide promoted angiogenesis and inhibited apoptosis and autophagy via activation of the Akt/eNOS pathway in $\mathrm{MCAO} / \mathrm{R}$ rats [317]. As for the BBB permeability, it was found that a significant level of bilobalide could be detected in the rat brain after administration of a single dose ( $8 \mathrm{mg} / \mathrm{kg}$, i.v.), indicating the brain uptake of bilobalide [324].

3.4.3. Diterpenoids. Neuroprotective diterpenoids include andrographolide [57, 325-328], erinacine A [329], ginkgolide $\mathrm{B}[38,72,330,331]$, ginkgolide $\mathrm{K}[11,332]$, pseudopterosin A [333], salvinorin A [334], tanshinone I [335], tanshinone IIA [75, 336-343], totarol [344], triptolide [113, 345, 346], and (1S,2E,4R,6R,-7E,11E)-2,7,11-cembratriene-4,6-diol [347].

Andrographolide is the primary bioactive compound in Andrographis paniculata, a traditional Chinese medicine that possesses anti-inflammatory, antiviral, and antibacterial activities [348]. Pharmacodynamic studies have shown that andrographolide could cross the $\mathrm{BBB}$, so it was extensively studied in various CNS disorders such as ischemic stroke, Alzheimer's disease, and multiple sclerosis [349]. Recently, andrographolide was subjected to a phase II clinical trial (NCT02280876) to evaluate its efficacy in multiple sclerosis. For ischemic stroke, andrographolide was reported to reduce cerebral infarction and neurological deficits in both the transient and permanent MCAO models. The neuroprotective strategies of andrographolide mainly involved antiinflammation via inhibition of NF- $\kappa \mathrm{B}$ and HIF- $1 \alpha$, antioxidation via upregulation of $\mathrm{Nrf} 2 / \mathrm{HO}-1$ and suppression of NOX2, and BBB protection [57, 325-327]. Besides, andrographolide also promoted long-term cognitive recovery in a global ischemia model by enhancing the BDNF/TrkB pathway [328]. Notably, andrographolide was able to cross BBB and possessed relatively low toxicity and high bioavailability, as only a single dose of $10 \mu \mathrm{g} / \mathrm{kg}$ (i.v.) was needed to exert neuroprotective activity against tMCAO/R injuries in rats [327]. Hereby, andrographolide might be a great candidate for ischemic stroke treatment.

Ginkgolides, another group of major terpenoids in EGb761, are diterpenoids and have been applied to clinical ischemic stroke treatment in China for a decade. Two intravenous injections that contain ginkgolides as active ingredients are approved as Chinese herbal medicine to treat mild to moderate cerebral infarction in China: ginkgolide injection and ginkgolide meglumine injection. Ginkgolide injection (approval number Z20110035), containing bilobalide, ginkgolide $\mathrm{A}$, ginkgolide $\mathrm{B}$, and ginkgolide $\mathrm{C}$, has been manufactured by Chengdu Baiyu Pharmaceutical Co., Ltd., since 2012. Ginkgolide meglumine injection (GMI, approval number Z20120024), produced by Jiangsu Kanion Pharmaceutical Co., Ltd., excludes bilobalide and only contains ginkgolide A $(1.6 \mathrm{mg} / \mathrm{mL})$, ginkgolide $\mathrm{B}$ $(2.9 \mathrm{mg} / \mathrm{mL})$, and ginkgolide $\mathrm{K}(0.19 \mathrm{mg} / \mathrm{mL})$ as active 
ingredients [350]. Recently, several studies reported the neuroprotective mechanism of GMI in animal models. To illustrate, Geng et al. reported that GMI improved energy metabolism, reduced oxidative stress, and maintained cerebral homeostasis using a metabolomic profiling method [350]. Another study revealed that GMI targeted the PI3K/Akt pathway to activate cytoprotective transcriptional factors such as Nrf2 and CREB [351]. Notably, the neuroprotective efficacy of GMI was found to be as strong as edaravone [351]. Apart from the injections, some pure ginkgolides were also extensively studied in ischemic stroke. For example, ginkgolide $\mathrm{B}$ was found to be a natural platelet-activating factor (PAF) receptor antagonist and inhibited the microglial activation via inhibition of the $\mathrm{PAF}$ receptor in $\mathrm{tMCAO} / \mathrm{R}$ mice [72]. Besides, ginkgolide $\mathrm{B}$ also possessed multiple neuroprotective strategies including anti-inflammation via suppression of NF- $\kappa \mathrm{B}$, antiapoptosis, antiexcitotoxicity, and BBB protection [38, 330, 331]. In addition, ginkgolide $\mathrm{K}$ was also reported to exert neuroprotection in MCAO models. The neuroprotective mechanisms of ginkgolide $\mathrm{K}$ involved antioxidation, mitochondrial protection, elevation of autophagy via upregulation of the AMPK/mTOR/ULK1 signaling pathway, and promotion of neurogenesis by activating JAK2/STAT3 [11, 332, 352, 353]. A study compared the neuroprotective efficacy of ginkgolides $(\mathrm{A}, \mathrm{B}$, and $\mathrm{K})$ and bilobalide and indicated that ginkgolide $\mathrm{B}$ exerted the strongest activities to reduce cerebral infarction and oxidative stress via the promotion of the Akt/Nrf2 pathway [354]. To conclude, ginkgolides and bilobalide have been primarily applied to clinical treatment, showing great potential in ischemic stroke.

Tanshinones are the major components in the root or rhizome of Salvia miltiorrhiza (Danshen), a Chinese medicine that is traditionally used to treat cardiovascular diseases. More than 40 tanshinones were found in the Danshen extract, with tanshinone I and tanshinone IIA being widely studied in the ischemic stroke area [355]. Tanshinone I was found to reduce neuronal death via anti-inflammation in global cerebral ischemic gerbils [335]. Tanshinone IIA was found to improve cerebral infarction and poststroke recovery regardless of administration before or after ischemia. The major mechanisms for tanshinone IIA-mediated neuroprotection were anti-inflammation and antiapoptosis. Antiinflammatory activity of tanshinone IIA was achieved via suppression of the proinflammatory cytokines HMGB1 and MIF, which then downregulated the MAPKs, upregulated the PPAR $\gamma$, and inhibited the astrocyte activation $[75,337$, $339,340,342,343]$. Tanshinone IIA-mediated antiapoptosis was reported to be regulated by activation of the PI3K/Akt pathway [336, 341]. In addition, tanshinone IIA improved neuronal survival and synaptic plasticity by promotion of the BDNF/CREB pathway and elevation of TORC1 (transducers of regulated CREB), a CREB coactivator [338]. Hereby, tanshinone IIA showed marked neuroprotective activity in experimental ischemic stroke. However, tanshinone IIA possessed poor solubility and half-life, limiting its BBB permeability. Accordingly, various methods were developed to enhance its bioavailability. As an example, Liu et al. developed a drug delivery system for tanshinone IIA, called cationic bovine serum albumin-conjugated tanshinone IIA PEGylated nanoparticles (CBSA-PEG-NPs). They indicated that CBSA-PEG-NPs increased the brain delivery efficiency of tanshinone IIA and thus enhanced its neuroprotective activity in ischemic stroke [339, 340].

Triptolide, a major bioactive diterpenoid in Tripterygium wilfordii, is famous for its anti-inflammatory and immunosuppressive activities. Studies showed that triptolide possessed good neuroprotective efficacy with very low effective doses (single dose of $0.2 \mathrm{mg} / \mathrm{kg}$, i.p.) in $\mathrm{tMCAO} / \mathrm{R}$ rats [345]. The major neuroprotective strategy of triptolide was anti-inflammation via inhibition of the p38/NF- $\kappa$ B pathway $[345,346]$. Besides, triptolide also lowered BBB permeability, suppressed apoptosis, and enhanced autophagy in the MCAO rats $[113,346]$. Yet, triptolide exhibited high toxicity on the liver and the heart, limiting its clinical application [356].

3.4.4. Triterpenoids. Triterpenoids are the most popular group of terpenoids and the major constituents of decoction and the extracts of many medical plants. Triterpenoid saponins, the glycosides of triterpenoids, are an important form of the bioactive triterpenoids. In total, 20 triterpenoids are found to exhibit neuroprotection in ischemic stroke including arjunolic acid [357], asiatic acid [358, 359], boswellic acids [81, 360, 361], 28-O-caffeoyl betulin [362], celastrol [73, 363], echinocystic acid [364], 18 $\beta$-glycyrrhetinic acid [365], maslinic acid [366], ursolic acid [367], and triterpenoid glycosides: madecassoside [368], astragaloside IV [62, $114,369,370]$, glycyrrhizin [91, 371-373], diammonium glycyrrhizinate [374], ginsenoside $\mathrm{Rb} 1$ [34, 55, 115, 375, 376], ginsenoside Rd [49, 377-383], ginsenoside Rg1 [384-389], ginsenoside Rg3 [390], pseudoginsenoside F11 [391, 392], and notoginsenoside R1 [393].

Boswellic acids are present in the gum resin of the herb Boswellia serrata. Several types of boswellic acids are found in B. serrata, and two of them, namely, acetyl-11-keto- $\beta$-boswellic acid (AKBA) and 11-keto- $\beta$-boswellic acid (KBA), were reported to exert neuroprotection in ischemic stroke [394]. The major neuroprotective strategies of AKBA involved anti-inflammation via inhibition of 5-LOX and promotion of Nrf2/HO-1-mediated antioxidation [81, 360]. Similar to AKBA, the activation of the Nrf2/HO-1 pathway was also observed in KBA-mediated neuroprotection [361]. Yet, boswellic acids were reported to have poor solubility and half-time, largely restricting their pharmacological applications. Efforts were made to develop high-efficiency delivery systems for boswellic acids. For example, Ding et al. reported an AKBA-loaded O-carboxymethyl chitosan nanoparticle system and found that this system enhanced the neuroprotective efficacy of AKBA [81].

Celastrol is another major bioactive terpenoid in the root of Tripterygium wilfordii, in addition to the diterpenoid triptolide. Celastrol exerted neuroprotection in ischemic stroke mainly via its anti-inflammatory activity. It is reported that celastrol promoted the polarization of microglia/macrophages from the proinflammatory M1 phase to the antiinflammatory M2 phase by decreasing proinflammatory cytokine IL-33 and its corresponding receptor ST2 (growth 
stimulation expressed gene 2) in pMCAO rats [73]. Besides, celastrol also inhibited the JNK/NF- $\kappa \mathrm{B}$ pathway to suppress the inflammatory cascade in the ischemic brain [363]. The neuroprotective efficacy of celastrol was relatively high, improving cerebral infarction and neurological deficits with low effective doses (1-3 mg/kg, i.p.). Yet, similar to triptolide, celastrol also had high toxicity and poor solubility problems, which limited its pharmacological applications [356].

Astragaloside IV is a saponin abundant in the dry root of Astragalus membranaceus (Huangqi), a Chinese herbal medicine that has been used to treat ischemic stroke in China for a long time [395]. Notably, astragaloside IV is usually regarded as a quality control marker of Huangqi. A meta-analysis study revealed that astragaloside IV reduced cerebral infarction, improved neurological impairments, decreased brain edema, and enhanced BBB integrity in experimental ischemic stroke [395]. The neuroprotective mechanisms of astragaloside IV were multiple including antioxidation, antiapoptosis, anti-inflammation, autophagy modulation, and mitochondrial protection $[62,114,369$, 370]. Hexokinase-II (HK-II), an enzyme for glycolysis, inhibited the MPTP opening after binding to mitochondria. Astragaloside IV was found to enhance the activity of HK-II and promote its binding to mitochondria, thus inhibiting mitochondrial dysfunction and mitochondrial apoptosis, as well as improving glycolysis to alleviate energy depletion [369]. Besides, astragaloside IV also activated the Nrf2 pathway to reduce oxidative stress and $\mathrm{BBB}$ permeability in an LPSinjured mouse model [62].

Ginseng, the roots of Panax ginseng, has been widely used in East Asian countries as a medication for thousands of years. Studies showed that ginsenosides are the major bioactive ingredients that contribute to the numerous therapeutic effects of ginseng. There are more than 180 kinds of ginsenosides extracted from ginseng. Generally, they can be divided into two groups: the protopanaxadiol group, including Rb1, $\mathrm{Rb} 2, \mathrm{Rd}$, and $\mathrm{Rg} 3$, and the protopanaxatriol group, including Rg1, Rf, and Re. Among them, ginsenosides Rb1, Rd, Rg1, and Rg3 were reported to exert neuroprotective activities in ischemic stroke [396].

Ginsenoside Rb1 (GS-Rb1) is the most abundant ginsenoside in ginseng, accounting for about $31 \%$ of all ginsenosides in Chinese/Korean ginseng. Wang et al. found that GS-Rb1 improved the abnormal microenvironment of the hippocampus in the photothrombotic cerebral ischemia model, as evidenced by the reduced excitotoxicity, intracellular $\mathrm{Ca}^{2+}$ level, and apoptosis, and improved regional cerebral blood flow. The mechanisms for those improvements are reported to be upregulation of GLT-1 and inhibition of NMDA receptors and cytochrome c release [34]. Besides, GS-Rb1 inhibited the NOX4-mediated ROS production and thus reduced $\mathrm{BBB}$ permeability due to downregulated MMP-9 activation [55]. Furthermore, GS-Rb1 was found to enhance cytoprotective autophagy, neurogenesis, and BDNF release in MCAO models [115, 376]. Notably, Dong et al. showed that GS-Rb1 also exerted neuroprotective activity in aged mice [375].

Ginsenoside Rd (GS-Rd) has been regarded as one of the important markers for the quality of ginseng. GS-Rd showed marked neuroprotective activity in animal models regardless of preconditioning or postconditioning. Besides, GS-Rd has a relatively wide therapeutic window. Ye et al. found that GSRd improved cerebral infarction and neurological outcomes even when administrated after $4 \mathrm{~h}$ of ischemia in MCAO rats [383]. The neuroprotective mechanisms of GS-Rd were multiple including anti-inflammation, antioxidation, and antimitochondrial apoptosis via inhibition of PARP-1 (poly(ADP-ribose) polymerase 1) [377, 379-382]. Besides, GS-Rd also inhibited DNA damage via upregulation of NEIL1/3 (human endonuclease VIII-like proteins) and reduced intracellular $\mathrm{Ca}^{2+}$ accumulation by suppression of TRPM7 and ASIC $[49,378]$. Most importantly, GS-Rd could effectively cross the intact $\mathrm{BBB}$ and was reported to have much stronger neuroprotective efficacy than edaravone [383]. Hereby, GS-Rd might possess great potential for clinical ischemic stroke treatment. Accordingly, GS-Rd was subjected to clinical trials, including a phase II trial (NCT00591084) and a phase III trial (NCT00815763). In total, 190 ischemic stroke patients in phase II and 390 patients in phase III were recruited. They were intravenously injected with GS-Rd $(10,20 \mathrm{mg})$ within $72 \mathrm{~h}$ after ischemic stroke onset for $14 \mathrm{~d}$ [382]. Clinical results showed that GSRd improved the NIHSS (National Institutes of Health Stroke Scale) at $15 \mathrm{~d}$, with no significantly elevated mortality or adverse effects [397]. Hence, GS-Rd is one of the most potential drug candidates for ischemic stroke treatment.

Ginsenoside Rg1 (GS-Rg1) accounts for about 23\% of all ginseng-derived ginsenosides in Chinese/Korean ginseng. It was indicated that GS-Rg1 possessed equivalent neuroprotective efficacy to GS-Rbl in $\mathrm{tMCAO} / \mathrm{R}$ rats [398]. The neuroprotective strategies of GS-Rg1 included anti-inflammation, antiexcitotoxicity, antioxidation via inhibition of miR-144, promotion of angiogenesis via activation of the PI3K/Akt/mTOR pathway, and upregulation of BDNF [384-386, 388]. In addition, GS-Rg1 also protected BBB integrity and reduced brain edema by inhibiting aquaporin-4, a water channel protein highly expressed in the astrocyte foot [387, 389]. Yet, the bioavailability, BBB permeability, and half-time of GS-Rg1 were poor, limiting its clinical application [399].

3.4.5. Tetraterpenoids. In total, 3 tetraterpenoids are reported to be neuroprotective in ischemic stroke, including astaxanthin [120, 400, 401], fucoxanthin [402], and lutein [403]. Notably, they all belong to the xanthophyll type of carotenoids.

Astaxanthin, a well-known antioxidant, exists abundantly in algal species, such as Haematococcus pluvialis, and crustaceans. As the only carotenoid that could cross the $\mathrm{BBB}$ according to the present studies, astaxanthin has received much attention in ischemic stroke research [404]. It was found that pretreatment with astaxanthin decreased cerebral infarction and neurological deficits in tMCAO/R rats via antioxidation and antiapoptosis [120, 400, 401]. Besides, astaxanthin also promoted neurogenesis and the release of neurotrophins such as BDNF and NGF [120, 401]. Notably, a clinical trial (NCT03945526) was conducted to test the effect of astaxanthin supplementation $(2 \times 8 \mathrm{mg}$ for 
$7 \mathrm{~d}$ ) on plasma MDA levels and neurological deficits of ischemic stroke patients.

3.4.6. Summary of Terpenoids. From the present studies, borneol, bilobalide, ginkgolides, and ginsenoside Rd have been preliminarily applied to clinical ischemic stroke treatment, and the effectiveness of ginsenoside $\mathrm{Rd}$ was further indicated by the clinical data. Besides, carvacrol, andrographolide, and astaxanthin were also great candidates for ischemic stroke treatment due to their high bioavailability and BBB permeability in rodent models. In addition, triptolide and celastrol showed marked neuroprotective efficacy and high bioavailability in experimental ischemic stroke. Yet, they possessed high toxicity, limiting their further clinical application. Generally, terpenoids exhibit strong neuroprotective activity in experimental ischemic stroke. However, the solubility and BBB permeability of terpenoids such as tanshinones, boswellic acids, and celastrol are poor. Although several strategies, such as the development of the nanoparticle delivery systems, have been tried to solve this limitation, no effective strategies have been officially approved at present.

3.5. Alkaloids. Alkaloids refer to a class of natural compounds that have one or more nitrogen atoms in the heterocyclic ring. Alkaloids can be produced in many species of plants, especially flowering plants, in the form of organic acids, esters, or binding with sugars and tannins rather than free bases. Totally, 19 natural alkaloids were found to exert neuroprotection after searching the research of the past 10 years, including berberine [74, 92, 405-409], boldine [410], capsaicin [47, 411], dihydrocapsaicin $[44,412-$ 414], harmine [35], higenamine [415], neferine [63], nicotine [416], levo-tetrahydropalmatine [417], oxymatrine [83, 418], oxysophoridine [419], piperine [420], rhynchophylline [421], sinomenine $[87,422,423]$, solasodine [424], sophoridine $[425,426]$, tetrandrine [427], trigonelline [428], and vinpocetine [93, 429]. Their neuroprotective mechanisms are explained in Table 8 , and the chemical structures of the extensively studied alkaloids are shown in Table 7.

Berberine is an isoquinoline alkaloid present in the Chinese medicine Rhizoma coptidis (Huanglian). It was found that berberine could cross the BBB and accumulate in the brain tissue, so it has been extensively studied in CNS disorders including ischemic stroke [409]. Berberine exerted neuroprotection in both the global and transient cerebral ischemia models via two major mechanisms: antiapoptosis and anti-inflammation. The antiapoptosis strategy was mainly achieved by activating the PI3K/Akt pathway [74, 405, 407]. Yang et al. found that berberine enhanced the expression of BDNF and its receptor TrkB to promote the activation of the PI3K/Akt pathway [407]. Other studies further indicated that berberine could promote the activity of the PI3K p55 $\gamma$ subunit and enhance Akt-mediated GSK activation to suppress neuronal apoptosis [405, 408]. As for the anti-inflammatory strategy, it was found that berberine reduced microglial and astrocyte activation and enhanced AMPK-dependent microglial M2 polarization [406, 409]. Besides, the
HMGB1/TLR4 pathway was also involved in inhibiting the activation of NF- $\kappa \mathrm{B}$ and the subsequent inflammatory cascade $[92,408]$. In addition, $\mathrm{Zhu}$ et al. also reported the role of berberine in promoting angiogenesis in $\mathrm{tMCAO} / \mathrm{R}$ mice [409]. Hereby, berberine showed marked neuroprotection in experimental ischemic stroke and possessed great potential for clinical application in ischemic stroke.

Capsaicin and dihydrocapsaicin are the main capsaicinoids that contribute to the pungency of chili peppers. Capsaicin and dihydrocapsaicin are famous TRPV1 antagonists, possessing desensitizing effects on TRPV1 [47]. Hence, capsaicin- and dihydrocapsaicin-mediated neuroprotection were mainly attributed to the inhibition of TRPV1. To illustrate, capsaicin was found to reduce neuronal and neurovascular damage via inhibition of TRPV1-induced excitotoxicity [47, 411]. Besides, inhibition of TRPV1 also led to hypothermia, a state that has been proved to have neuroprotective effects in experimental ischemic stroke [413]. Hereby, dihydrocapsaicin has been shown to possess marked neuroprotection via pharmacological induction of hypothermia in $\mathrm{MCAO} / \mathrm{R}$ models [44, 413]. It was reported that dihydrocapsaicin-induced hypothermia protected the ischemic brain through multiple mechanisms, including antiapoptosis via activation of the PI3K/Akt pathway and promotion of Nrf2-mediated antioxidation and antiinflammation [412-414]. Yet, it was indicated that the bioavailability and half-time of capsaicin and dihydrocapsaicin were low, limiting their clinical application. Hence, researchers are searching for the proper delivery system for capsaicin and dihydrocapsaicin.

Sinomenine is the major bioactive ingredient in the herb Sinomenium acutum. Sinomenine is known for its immunosuppressive activity and has been used to treat rheumatoid arthritis in China [87]. For neuroprotection, sinomenine was found to reduce cerebral infarction, neurological deficits, and brain edema in tMCAO/R rodents via its antiinflammatory activity [87, 422, 423]. Qiu et al. reported that sinomenine upregulated AMPK to inhibit the activation of the NLRP3 (NOD-like receptor pyrin 3) inflammasome, an activator for the release of proinflammatory cytokines [422]. Besides, it was shown that sinomenine also promoted the activation of DRD2 (astrocytic dopamine D2 receptor), an anti-inflammatory factor in astrocytes, and the expression and nuclear translocation of CRYAB $(\alpha \mathrm{B}-$ crystallin), a heat shock protein that is regulated by DRD2 [87]. Furthermore, sinomenine inhibited cerebral $\mathrm{I} / \mathrm{R}$-induced acidosis and intracellular $\mathrm{Ca}^{2+}$ accumulation in $\mathrm{tMCAO} / \mathrm{R}$ rats by suppressing ASICla and L-type calcium channels $[423,430]$. Notably, sinomenine was able to cross the BBB as indicated by Wu et al. that $0.11 \mu \mathrm{g} / \mathrm{g}$ was accumulated in the rat brain after $0.5 \mathrm{~h}$ administration of sinomenine ( $10 \mathrm{mg} / \mathrm{kg}$, i.p.) [430].

Vinpocetine is naturally present in the periwinkle plant and has been investigated at length for its effect against ischemic stroke. The neuroprotective strategy of vinpocetine in ischemic stroke mainly involved anti-inflammation via inhibition of the TLR4/MyD88 pathway [93, 429]. Notably, vinpocetine also showed a neuroprotective effect in a phase II clinical trial (NCT02878772), in which 60 
TABLE 8: Neuroprotective alkaloids and their functional mechanisms and targets ${ }^{\mathrm{e}}$.

\begin{tabular}{|c|c|c|}
\hline Compounds & Mechanisms and targets & Ref. \\
\hline \multicolumn{3}{|l|}{ Alkaloids (19) } \\
\hline Berberine & $\begin{array}{c}\text { Antiapoptosis: BDNF-TrkB-PI3K/Akt } \uparrow, \text { PI } 3 \mathrm{~K} \text { p } 55 \gamma \text { activity } \uparrow, \text { Akt/GSK } \uparrow \text {; } \\
\text { angiogenesis } \uparrow \text {; claudin-5 } \uparrow \text {; anti-inflammation: microglial and astrocyte } \\
\text { activation } \downarrow, \text { AMPK-dependent microglial M2 polarization } \uparrow \\
\text { HMGB1/TLR4/NF- } \kappa \mathrm{B} \downarrow\end{array}$ & {$[74,92,405-409]$} \\
\hline Boldine & Anti-inflammation & {$[410]$} \\
\hline Capsaicin & $\begin{array}{c}\text { Antiexcitotoxicity: TRPV1-dependent inhibition of NMDA receptors } \uparrow ; \\
\text { neurovascular protection }\end{array}$ & {$[47,411]$} \\
\hline Dihydrocapsaicin & $\begin{array}{c}\text { Hypothermia: TRPV1 } \downarrow ; \text { PI3K/Akt } \uparrow \text {; BBB protection; antioxidation; } \\
\text { anti-inflammation }\end{array}$ & {$[44,412-414]$} \\
\hline Harmine & GLT-1 $\uparrow$; astrocyte activation $\downarrow$ & {$[35]$} \\
\hline Higenamine & 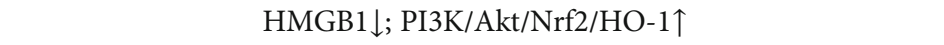 & {$[415]$} \\
\hline Neferine & Mitochondrial protection: Nrf2 pathway $\uparrow$ & {$[63]$} \\
\hline Nicotine & Anti-inflammation: microglial proliferation $\downarrow, \alpha 7 \mathrm{nAchR} \uparrow$ & [416] \\
\hline Levo-tetrahydropalmatine & Antiapoptosis: c-Abl $\downarrow$ & [417] \\
\hline Oxymatrine & $\begin{array}{c}\text { Anti-inflammation: arachidonic acid release } \downarrow, 12 / 15-\mathrm{LOX} / \mathrm{p} 38 \\
\text { MAPK/cPLA2 } \downarrow ; \text { Nrf2/HO- } 1 \uparrow\end{array}$ & {$[83,418]$} \\
\hline Oxysophoridine & Antiapoptosis & [419] \\
\hline Sophoridine & Antiapoptosis; ASIC1 $\downarrow$; TRAF6 $\downarrow / E r k 1 / 2 \uparrow$ & {$[425,426]$} \\
\hline Piperine & Anti-inflammation & [420] \\
\hline Rhynchophylline & $\mathrm{PI} 3 \mathrm{~K} / \mathrm{Akt} / \mathrm{mTOR} \uparrow$ & {$[421]$} \\
\hline Sinomenine & $\begin{array}{c}\text { Anti-inflammation: NLRP3 inflammasomes } \downarrow \text {, DRD2 } \uparrow / C R Y A B \uparrow / S T A T 3 \downarrow ; \\
\text { AMPK } \uparrow ; \text { acidosis } \downarrow: \text { ASIC1a } \downarrow\end{array}$ & {$[87,422,423]$} \\
\hline Solasodine & Antioxidation & {$[424]$} \\
\hline Tetrandrine & 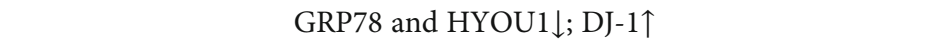 & [427] \\
\hline Trigonelline & Glutathione-mediated myeloperoxidase expression $\downarrow$ & [428] \\
\hline Vinpocetine & Anti-inflammation: TLR4/MyD88/NF- $\kappa \mathrm{B} \downarrow$ & {$[93,429]$} \\
\hline
\end{tabular}

e Notes: $\uparrow$ : activation or upregulation; $\downarrow$ : inhibition or downregulation. Abbreviations do not appear in the text. c-Abl: nonreceptor Abelson tyrosine kinase; DJ1: PARK7, Parkinsonism associated deglycase; GRP78: glucose-regulated protein of $78 \mathrm{kDa}$; HYU1: hypoxia upregulated protein 1.

ischemic stroke patients were divided into two groups: half of the patients only received standard treatment, while the other half of the patients received $30 \mathrm{mg} / \mathrm{d}$ (i.v. for $14 \mathrm{~d}$ ) vinpocetine treatment plus standard treatment [431]. Results showed that vinpocetine reduced the secondary infarction enlargement and NF- $\kappa \mathrm{B}$-mediated inflammation and improved poststroke neurological functional recovery [431]. More importantly, vinpocetine had high BBB permeability, greatly enhancing its bioavailability in CNS disorders [431]. Accordingly, vinpocetine might be one of the most promising candidates for ischemic stroke treatment.

In summary, vinpocetine and berberine had high BBB permeability and showed great neuroprotective efficacy in experimental ischemic stroke. Besides, vinpocetine also showed neuroprotective activity in the phase II clinical trial. Hence, vinpocetine and berberine might possess great potential in clinical ischemic stroke treatment. As for capsaicin and dihydrocapsaicin, although they showed good neuroprotective efficacy in experimental ischemic stroke, their poor bioavailability and BBB permeability might limit their further clinical applications.

\section{Conclusion}

Phytochemicals have been well studied in experimental ischemic stroke due to their marked neuroprotective activities. In this review, we listed 148 phytochemicals that were reported to exhibit neuroprotection in various animal models of ischemic stroke, including flavonoids (46), stilbenoids (7), other phenols (20), terpenoids (56), and alkaloids (19). Notably, several phytochemicals have been primarily applied in clinical ischemic stroke treatment or have shown neuroprotective activities in clinical trials. Those phytochemicals include scutellarin, pinocembrin, puerarin, hydroxysafflor yellow A, salvianolic acids, rosmarinic acid, borneol, bilobalide, ginkgolides, ginsenoside Rd, and vinpocetine. However, the clinical application was mainly carried out in China with purified/concentrated plant extracts or a mixture of several compounds. In addition, many phytochemicals, such as baicalein and baicalin, CBD, carvacrol, andrographolide, astaxanthin, and berberine, showed great neuroprotective efficacy and high BBB permeability and bioavailability in experimental ischemic stroke research. Hence, they also possessed great potential for clinical application. However, other 
agents such as naringenin, curcumin, EGCG, capsaicin, dihydrocapsaicin, and tanshinone IIA exhibited marked neuroprotective efficacy in experimental ischemic stroke but had poor solubility and BBB permeability. For those phytochemicals, modification of their chemical structures or development of efficient drug delivery systems is needed to enhance their BBB permeability.

\section{Conflicts of Interest}

The authors declare no conflicts of interest.

\section{Acknowledgments}

This work was partially supported by the HKU Seed Fund for Translational and Applied Research (201811160003).

\section{References}

[1] G. Chauhan and S. Debette, "Genetic risk factors for ischemic and hemorrhagic stroke," Current Cardiology Reports, vol. 18, no. 12, p. 124, 2016.

[2] A. Durukan and T. Tatlisumak, "Acute ischemic stroke: overview of major experimental rodent models, pathophysiology, and therapy of focal cerebral ischemia," Pharmacology Biochemistry and Behavior, vol. 87, no. 1, pp. 179-197, 2007.

[3] E. S. Donkor, "Stroke in the Century: A Snapshot of the Burden, Epidemiology, and Quality of Life," Stroke Research and Treatment, vol. 2018, Article ID 3238165, 10 pages, 2018.

[4] C. Xing, K. Arai, E. H. Lo, and M. Hommel, "Pathophysiologic cascades in ischemic stroke," International Journal of Stroke, vol. 7, no. 5, pp. 378-385, 2012.

[5] E. H. Lo, T. Dalkara, and M. A. Moskowitz, "Mechanisms, challenges and opportunities in stroke," Nature Reviews Neuroscience, vol. 4, no. 5, pp. 399-414, 2003.

[6] A. Kunz, U. Dirnagl, and P. Mergenthaler, "Acute pathophysiological processes after ischaemic and traumatic brain injury," Best Practice \& Research. Clinical Anaesthesiology, vol. 24, no. 4, pp. 495-509, 2010.

[7] M. Y. Wu, G. T. Yiang, W. T. Liao et al., "Current mechanistic concepts in ischemia and reperfusion injury," Cellular Physiology and Biochemistry, vol. 46, no. 4, pp. 1650-1667, 2018.

[8] J. Minnerup, B. A. Sutherland, A. M. Buchan, and C. Kleinschnitz, "Neuroprotection for stroke: current status and future perspectives," International Journal of Molecular Sciences, vol. 13, no. 12, pp. 11753-11772, 2012.

[9] J. C. Zhang, H. Xu, Y. Yuan et al., "Delayed treatment with green tea polyphenol EGCG promotes neurogenesis after ischemic stroke in adult mice," Molecular Neurobiology, vol. 54, no. 5, pp. 3652-3664, 2017.

[10] S. Liu, Y. Cao, M. Qu et al., "Curcumin protects against stroke and increases levels of Notch intracellular domain," Neurological Research, vol. 38, no. 6, pp. 553-559, 2016.

[11] M. Chen, W. Zou, M. Chen et al., "Ginkgolide K promotes angiogenesis in a middle cerebral artery occlusion mouse model via activating JAK2/STAT3 pathway," European Journal of Pharmacology, vol. 833, pp. 221-229, 2018.

[12] L. Catanese, J. Tarsia, and M. Fisher, "Acute ischemic stroke therapy overview," Circulation Research, vol. 120, no. 3, pp. 541-558, 2017.
[13] S. Bansal, K. S. Sangha, and P. Khatri, "Drug treatment of acute ischemic stroke," American Journal of Cardiovascular Drugs, vol. 13, no. 1, pp. 57-69, 2013.

[14] M. G. Lansberg, M. J. O'Donnell, P. Khatri et al., “Antithrombotic and thrombolytic therapy for ischemic stroke: antithrombotic therapy and prevention of thrombosis, 9th ed: American College of Chest Physicians evidence-based clinical practice guidelines," Chest, vol. 141, no. 2, pp. e601S-e636S, 2012.

[15] N. Kapil, Y. H. Datta, N. Alakbarova et al., "Antiplatelet and anticoagulant therapies for prevention of ischemic stroke," Clinical and Applied Thrombosis/Hemostasis, vol. 23, no. 4, pp. 301-318, 2017.

[16] A. Moretti, F. Ferrari, and R. F. Villa, "Neuroprotection for ischaemic stroke: current status and challenges," Pharmacology \& Therapeutics, vol. 146, pp. 23-34, 2015.

[17] O. Y. Bang, "Neuroprotective strategies for acute ischemic stroke: recent progress and future perspectives," Precision of Future Medicine, vol. 1, no. 3, pp. 115-121, 2017.

[18] K. Watanabe, M. Tanaka, S. Yuki, M. Hirai, and Y. Yamamoto, "How is edaravone effective against acute ischemic stroke and amyotrophic lateral sclerosis?," Journal of Clinical Biochemistry and Nutrition, vol. 62, no. 1, pp. 20-38, 2018.

[19] S. Kobayashi, S. Fukuma, T. Ikenoue, S. Fukuhara, S. Kobayashi, and on behalf of the Japan Stroke Data Bank, "Effect of edaravone on neurological symptoms in realworld patients with acute ischemic stroke," Stroke, vol. 50, no. 7, pp. 1805-1811, 2019.

[20] S. Kono, K. Deguchi, N. Morimoto et al., "Intravenous thrombolysis with neuroprotective therapy by edaravone for ischemic stroke patients older than 80 years of age," Journal of Stroke and Cerebrovascular Diseases, vol. 22, no. 7, pp. 1175-1183, 2013.

[21] M. Enomoto, A. Endo, H. Yatsushige, K. Fushimi, and Y. Otomo, "Clinical effects of early edaravone use in acute ischemic stroke patients treated by endovascular reperfusion therapy," Stroke, vol. 50, no. 3, pp. 652-658, 2019.

[22] Z. Q. Xu, Y. Zhou, B. Z. Shao, J. J. Zhang, and C. Liu, "A systematic review of neuroprotective efficacy and safety of Dl-3n-butylphthalide in ischemic stroke," The American Journal of Chinese Medicine, vol. 47, no. 3, pp. 507-525, 2019.

[23] S. Wang, F. Ma, L. Huang et al., "Dl-3-n-butylphthalide (NBP): a promising therapeutic agent for ischemic stroke," CNS \& Neurological Disorders Drug Targets, vol. 17, no. 5, pp. 338-347, 2018.

[24] R. G. Robinson, W. J. Shoemaker, M. Schlumpf, T. Valk, and F. E. Bloom, "Effect of experimental cerebral infarction in rat brain on catecholamines and behaviour," Nature, vol. 255, no. 5506, pp. 332-334, 1975.

[25] C. J. Sommer, "Ischemic stroke: experimental models and reality," Acta Neuropathologica, vol. 133, no. 2, pp. 245261, 2017.

[26] M. Gennaro, A. Mattiello, and T. Pizzorusso, "Rodent models of developmental ischemic stroke for translational research: strengths and weaknesses," Neural Plasticity, vol. 2019, 16 pages, 2019.

[27] M. Mehra, N. Henninger, J. A. Hirsch, J. Chueh, A. K. Wakhloo, and M. J. Gounis, "Preclinical acute ischemic stroke modeling," Journal of NeuroInterventional Surgery, vol. 4, no. 4, pp. 307-313, 2012. 
[28] C. Kleinschnitz, F. Fluri, and M. Schuhmann, "Animal models of ischemic stroke and their application in clinical research," Drug Design, Development and Therapy, vol. 9, pp. 3445-3454, 2015.

[29] Y. Ma, L. Li, L. Kong et al., "Pinocembrin protects bloodbrain barrier function and expands the therapeutic time window for tissue-type plasminogen activator treatment in a rat thromboembolic stroke model," BioMed Research International, vol. 2018, Article ID 8943210, 13 pages, 2018.

[30] R. J. Traystman, "Animal models of focal and global cerebral ischemia," ILAR Journal, vol. 44, no. 2, pp. 85-95, 2003.

[31] K. P. Doyle, R. P. Simon, and M. P. Stenzel-Poore, "Mechanisms of ischemic brain damage," Neuropharmacology, vol. 55, no. 3, pp. 310-318, 2008.

[32] U. Dirnagl, C. Iadecola, and M. A. Moskowitz, "Pathobiology of ischaemic stroke: an integrated view," Trends in Neurosciences, vol. 22, no. 9, pp. 391-397, 1999.

[33] W. Krzyzanowska, B. Pomierny, M. Filip, and J. Pera, "Glutamate transporters in brain ischemia: to modulate or not?," Acta Pharmacologica Sinica, vol. 35, no. 4, pp. 444-462, 2014.

[34] S. Wang, M. Li, Y. Guo et al., "Effects of Panax notoginseng ginsenoside $\mathrm{Rb} 1$ on abnormal hippocampal microenvironment in rats," Journal of Ethnopharmacology, vol. 202, pp. 138-146, 2017.

[35] P. Sun, S. Zhang, Y. Li, and L. Wang, "Harmine mediated neuroprotection via evaluation of glutamate transporter 1 in a rat model of global cerebral ischemia," Neuroscience Letters, vol. 583, pp. 32-36, 2014.

[36] D. Amantea and G. Bagetta, "Excitatory and inhibitory amino acid neurotransmitters in stroke: from neurotoxicity to ischemic tolerance," Current Opinion in Pharmacology, vol. 35, pp. 111-119, 2017.

[37] J. Ding, G. Fu, Y. Zhao et al., "EGCG ameliorates the suppression of long-term potentiation induced by ischemia at the Schaffer collateral-CA1 synapse in the rat," Cellular and Molecular Neurobiology, vol. 32, no. 2, pp. 267-277, 2012.

[38] Z. Z. Yang, J. Li, S. X. Li, W. Feng, and H. Wang, "Effect of ginkgolide B on striatal extracellular amino acids in middle cerebral artery occluded rats," Journal of Ethnopharmacology, vol. 136, no. 1, pp. 117-122, 2011.

[39] Q. J. Wu and M. Tymianski, "Targeting NMDA receptors in stroke: new hope in neuroprotection," Molecular Brain, vol. 11, no. 1, p. 15, 2018.

[40] B. Ballarin and M. Tymianski, "Discovery and development of NA-1 for the treatment of acute ischemic stroke," Acta Pharmacologica Sinica, vol. 39, no. 5, pp. 661-668, 2018.

[41] B. Huang, P. Chen, L. Huang et al., "Geniposide attenuates post-ischaemic neurovascular damage via GluN2A/AKT/ERK-dependent mechanism," Cellular Physiology and Biochemistry, vol. 43, no. 2, pp. 705-716, 2017.

[42] H. S. Sun, "Role of TRPM7 in cerebral ischaemia and hypoxia," The Journal of Physiology, vol. 595, no. 10, pp. 30773083, 2017.

[43] C. Guo, Y. Ma, S. Ma et al., "The role of TRPC6 in the neuroprotection of calycosin against cerebral ischemic injury," Scientific Reports, vol. 7, no. 1, p. 3039, 2017.

[44] Z. Cao, A. Balasubramanian, S. E. Pedersen, J. Romero, R. G. Pautler, and S. P. Marrelli, "TRPV1-mediated pharmacological hypothermia promotes improved functional recovery following ischemic stroke," Scientific Reports, vol. 7, no. 1, article 17685, 2017.
[45] Y. Lin, F. Chen, J. Zhang et al., "Neuroprotective effect of resveratrol on ischemia/reperfusion injury in rats through TRPC6/CREB pathways," Journal of Molecular Neuroscience, vol. 50, no. 3, pp. 504-513, 2013.

[46] W. Chen, B. Xu, A. Xiao et al., "TRPM7 inhibitor carvacrol protects brain from neonatal hypoxic-ischemic injury," Molecular Brain, vol. 8, no. 1, p. 11, 2015.

[47] N. H. Khatibi, V. Jadhav, S. Charles et al., "Capsaicin pretreatment provides neurovascular protection against neonatal hypoxic-ischemic brain injury in rats," Acta Neurochirurgica. Supplement, vol. 111, pp. 225-230, 2011.

[48] S. Vullo and S. Kellenberger, "A molecular view of the function and pharmacology of acid-sensing ion channels," Pharmacological Research, vol. 154, article 104166, 2020.

[49] Y. Zhang, L. Zhou, X. Zhang, J. Bai, M. Shi, and G. Zhao, "Ginsenoside-Rd attenuates TRPM7 and ASIC1a but promotes ASIC2a expression in rats after focal cerebral ischemia," Neurological Sciences, vol. 33, no. 5, pp. 1125-1131, 2012.

[50] T. H. Sanderson, C. A. Reynolds, R. Kumar, K. Przyklenk, and M. Huttemann, "Molecular mechanisms of ischemiareperfusion injury in brain: pivotal role of the mitochondrial membrane potential in reactive oxygen species generation," Molecular Neurobiology, vol. 47, no. 1, pp. 9-23, 2013.

[51] C. L. Allen and U. Bayraktutan, "Oxidative stress and its role in the pathogenesis of ischaemic stroke," International Journal of Stroke, vol. 4, no. 6, pp. 461-470, 2009.

[52] S. E. Lakhan, A. Kirchgessner, D. Tepper, and A. Leonard, "Matrix metalloproteinases and blood-brain barrier disruption in acute ischemic stroke," Frontiers in Neurology, vol. 4, p. 32, 2013.

[53] T. Kahles and R. P. Brandes, "NADPH oxidases as therapeutic targets in ischemic stroke," Cellular and Molecular Life Sciences, vol. 69, no. 14, pp. 2345-2363, 2012.

[54] Y. Dai, H. Zhang, J. Zhang, and M. Yan, "Isoquercetin attenuates oxidative stress and neuronal apoptosis after ischemia/reperfusion injury via Nrf2-mediated inhibition of the NOX4/ROS/NF- $\kappa \mathrm{B}$ pathway," Chemico-Biological Interactions, vol. 284, pp. 32-40, 2018.

[55] W. Chen, Y. Guo, W. Yang, P. Zheng, J. Zeng, and W. Tong, "Protective effect of ginsenoside $\mathrm{Rb} 1$ on integrity of bloodbrain barrier following cerebral ischemia," Experimental Brain Research, vol. 233, no. 10, pp. 2823-2831, 2015.

[56] L. Zhai, M. Liu, T. Wang, H. Zhang, S. Li, and Y. Guo, "Picroside II protects the blood-brain barrier by inhibiting the oxidative signaling pathway in cerebral ischemia-reperfusion injury," PLoS One, vol. 12, no. 4, article e0174414, 2017.

[57] T. L. Yen, R. J. Chen, T. Jayakumar et al., “Andrographolide stimulates p38 mitogen-activated protein kinase-nuclear factor erythroid-2-related factor 2-heme oxygenase 1 signaling in primary cerebral endothelial cells for definite protection against ischemic stroke in rats," Translational Research, vol. 170, pp. 57-72, 2016.

[58] E. Birben, U. M. Sahiner, C. Sackesen, S. Erzurum, and O. Kalayci, "Oxidative stress and antioxidant defense," World Allergy Organization Journal, vol. 5, no. 1, pp. 9-19, 2012.

[59] L. Liu, L. M. Locascio, and S. Dore, "Critical role of Nrf2 in experimental ischemic stroke," Frontiers in Pharmacology, vol. 10, p. 153, 2019.

[60] L. Zhang, X. Zhang, C. Zhang et al., "Nobiletin promotes antioxidant and anti-inflammatory responses and elicits 
protection against ischemic stroke in vivo," Brain Research, vol. 1636, pp. 130-141, 2016.

[61] K. Wang, Z. Chen, J. Huang et al., "Naringenin prevents ischaemic stroke damage via anti-apoptotic and anti-oxidant effects," Clinical and Experimental Pharmacology \& Physiology, vol. 44, no. 8, pp. 862-871, 2017.

[62] H. Li, P. Wang, F. Huang et al., "Astragaloside IV protects blood-brain barrier integrity from LPS-induced disruption via activating Nrf2 antioxidant signaling pathway in mice," Toxicology and Applied Pharmacology, vol. 340, pp. 58-66, 2018.

[63] C. Wu, J. Chen, R. Yang, F. Duan, S. Li, and X. Chen, "Mitochondrial protective effect of neferine through the modulation of nuclear factor erythroid 2-related factor 2 signalling in ischaemic stroke," British Journal of Pharmacology, vol. 176, no. 3, pp. 400-415, 2019.

[64] R. Liu, M.-X. Pan, J.-C. Tang et al., "Role of neuroinflammation in ischemic stroke," Neuroimmunol Neuroinflamm, vol. 4, no. 8, pp. 158-166, 2017.

[65] M. Ahmad, N. Dar, Z. Bhat et al., "Inflammation in ischemic stroke: mechanisms, consequences and possible drug targets," CNS \& Neurological Disorders Drug Targets, vol. 13, no. 8, pp. 1378-1396, 2014.

[66] R. L. Jayaraj, S. Azimullah, R. Beiram, F. Y. Jalal, and G. A. Rosenberg, "Neuroinflammation: friend and foe for ischemic stroke," Journal of Neuroinflammation, vol. 16, no. 1, p. 142, 2019.

[67] M. Fang, Y. Yuan, P. Rangarajan et al., "Scutellarin regulates microglia-mediated TNC1 astrocytic reaction and astrogliosis in cerebral ischemia in the adult rats," BMC Neuroscience, vol. 16, no. 1, p. 84, 2015.

[68] C. C. Leonardo, M. Agrawal, N. Singh, J. R. Moore, S. Biswal, and S. Dore, "Oral administration of the flavanol (-)-epicatechin bolsters endogenous protection against focal ischemia through the Nrf2 cytoprotective pathway," The European Journal of Neuroscience, vol. 38, no. 11, pp. 3659-3668, 2013.

[69] M. Gelderblom, F. Leypoldt, J. Lewerenz et al., "The flavonoid fisetin attenuates postischemic immune cell infiltration, activation and infarct size after transient cerebral middle artery occlusion in mice," Journal of Cerebral Blood Flow and Metabolism, vol. 32, no. 5, pp. 835-843, 2012.

[70] Y. Wang, Q. Ren, X. Zhang, H. Lu, and J. Chen, "Neuroprotective mechanisms of calycosin against focal cerebral ischemia and reperfusion injury in rats," Cellular Physiology and Biochemistry, vol. 45, no. 2, pp. 537-546, 2018.

[71] X. Liu, S. Wen, F. Yan et al., "Salidroside provides neuroprotection by modulating microglial polarization after cerebral ischemia," Journal of Neuroinflammation, vol. 15, no. 1, p. 39, 2018.

[72] Z. M. Shu, X. D. Shu, H. Q. Li et al., "Ginkgolide B protects against ischemic stroke via modulating microglia polarization in mice," CNS Neuroscience \& Therapeutics, vol. 22, no. 9, pp. 729-739, 2016.

[73] M. Jiang, X. Liu, D. Zhang et al., "Celastrol treatment protects against acute ischemic stroke-induced brain injury by promoting an IL-33/ST2 axis-mediated microglia/macrophage M2 polarization," Journal of Neuroinflammation, vol. 15, no. 1, p. 78, 2018.

[74] M. Kim, M. S. Shin, J. M. Lee et al., "Inhibitory effects of isoquinoline alkaloid berberine on ischemia-induced apoptosis via activation of phosphoinositide 3-kinase/protein kinase B signaling pathway," International Neurourology Journal, vol. 18, no. 3, pp. 115-125, 2014.

[75] L. Zhou, S. C. Bondy, L. Jian et al., "Tanshinone IIA attenuates the cerebral ischemic injury-induced increase in levels of GFAP and of caspases-3 and -8," Neuroscience, vol. 288, pp. 105-111, 2015.

[76] C. Rink and S. Khanna, "Significance of brain tissue oxygenation and the arachidonic acid cascade in stroke," Antioxidants \& Redox Signaling, vol. 14, no. 10, pp. 1889-1903, 2011.

[77] S. K. Ha, P. Lee, J. A. Park et al., "Apigenin inhibits the production of $\mathrm{NO}$ and $\mathrm{PGE}_{2}$ in microglia and inhibits neuronal cell death in a middle cerebral artery occlusion-induced focal ischemia mice model," Neurochemistry International, vol. 52, no. 4-5, pp. 878-886, 2008.

[78] Y. Yao, L. Chen, J. Xiao et al., "Chrysin protects against focal cerebral ischemia/reperfusion injury in mice through attenuation of oxidative stress and inflammation," International Journal of Molecular Sciences, vol. 15, no. 11, pp. 2091320926, 2014.

[79] T. Wang, L. Zhai, H. Zhang, L. Zhao, and Y. Guo, "Picroside II inhibits the MEK-ERK1/2-COX2 signal pathway to prevent cerebral ischemic injury in rats," Journal of Molecular Neuroscience, vol. 57, no. 3, pp. 335-351, 2015.

[80] G. Liang, B. Shi, W. Luo, and J. Yang, "The protective effect of caffeic acid on global cerebral ischemia-reperfusion injury in rats," Behavioral and Brain Functions, vol. 11, no. 1, p. 18, 2015.

[81] Y. Ding, Y. Qiao, M. Wang et al., "Enhanced neuroprotection of Acetyl-11-Keto- $\beta$-Boswellic acid (AKBA)-loaded Ocarboxymethyl chitosan nanoparticles through antioxidant and anti-inflammatory pathways," Molecular Neurobiology, vol. 53, no. 6, pp. 3842-3853, 2016.

[82] L. Cui, X. Zhang, R. Yang et al., "Baicalein is neuroprotective in rat MCAO model: role of 12/15-lipoxygenase, mitogenactivated protein kinase and cytosolic phospholipase A2," Pharmacology, Biochemistry, and Behavior, vol. 96, no. 4, pp. 469-475, 2010.

[83] L. Cui, X. Zhang, R. Yang et al., "Neuroprotection and underlying mechanisms of oxymatrine in cerebral ischemia of rats," Neurological Research, vol. 33, no. 3, pp. 319-324, 2011.

[84] J. Yi, S. Park, R. Kapadia, and R. Vemuganti, "Role of transcription factors in mediating post-ischemic cerebral inflammation and brain damage," Neurochemistry International, vol. 50, no. 7-8, pp. 1014-1027, 2007.

[85] X. Bai, X. Zhang, L. Chen et al., "Protective effect of naringenin in experimental ischemic stroke: down-regulated NOD2, RIP2, NF- $\kappa$ B, MMP-9 and up-regulated claudin-5 expression," Neurochemical Research, vol. 39, no. 8, pp. 1405-1415, 2014.

[86] K. Zhou, J. Chen, J. Wu et al., “Atractylenolide III ameliorates cerebral ischemic injury and neuroinflammation associated with inhibiting JAK2/STAT3/Drp1-dependent mitochondrial fission in microglia," Phytomedicine, vol. 59, p. 152922, 2019.

[87] J. Qiu, Z. Yan, K. Tao et al., "Sinomenine activates astrocytic dopamine D2 receptors and alleviates neuroinflammatory injury via the CRYAB/STAT3 pathway after ischemic stroke in mice," Journal of Neuroinflammation, vol. 13, no. 1, p. 263, 2016.

[88] J. Pan, J. L. Jin, H. M. Ge et al., "Malibatol A regulates microglia M1/M2 polarization in experimental stroke in a $\operatorname{PPAR} \gamma$ - 
dependent manner," Journal of Neuroinflammation, vol. 12, no. 1 , p. $51,2015$.

[89] D. Xiong, Y. Deng, B. Huang et al., "Icariin attenuates cerebral ischemia-reperfusion injury through inhibition of inflammatory response mediated by NF- $\kappa \mathrm{B}, \operatorname{PPAR} \alpha$ and PPAR $\gamma$ in rats," International Immunopharmacology, vol. 30, pp. 157-162, 2016.

[90] Y. Wang, P. Ge, and Y. Zhu, "TLR2 and TLR4 in the brain injury caused by cerebral ischemia and reperfusion," Mediators of Inflammation, vol. 2013, Article ID 124614, 8 pages, 2013.

[91] J. Zhang, Y. Wu, Z. Weng, T. Zhou, T. Feng, and Y. Lin, “Glycyrrhizin protects brain against ischemia-reperfusion injury in mice through HMGB1-TLR4-IL-17A signaling pathway," Brain Research, vol. 1582, pp. 176-186, 2014.

[92] J. R. Zhu, H. D. Lu, C. Guo et al., "Berberine attenuates ischemia-reperfusion injury through inhibiting HMGB1 release and NF- $\kappa$ B nuclear translocation," Acta Pharmacologica Sinica, vol. 39, no. 11, pp. 1706-1715, 2018.

[93] L. R. Wu, L. Liu, X. Y. Xiong et al., "Vinpocetine alleviate cerebral ischemia/reperfusion injury by down-regulating TLR4/MyD88/NF- $\kappa \mathrm{B}$ signaling," Oncotarget, vol. 8, no. 46, pp. 80315-80324, 2017.

[94] X. K. Tu, W. Z. Yang, S. S. Shi et al., "Baicalin inhibits TLR2/4 signaling pathway in rat brain following permanent cerebral ischemia," Inflammation, vol. 34, no. 5, pp. 463-470, 2011.

[95] H. Qiao, X. Zhang, C. Zhu et al., "Luteolin downregulates TLR4, TLR5, NF- $\kappa$ B and p-p38MAPK expression, upregulates the p-ERK expression, and protects rat brains against focal ischemia," Brain Research, vol. 1448, pp. 71-81, 2012.

[96] L. Huang, C. Chen, X. Zhang et al., "Neuroprotective effect of curcumin against cerebral ischemia-reperfusion via mediating autophagy and inflammation," Journal of Molecular Neuroscience, vol. 64, no. 1, pp. 129-139, 2018.

[97] P. H. Chan, "Mitochondrial dysfunction and oxidative stress as determinants of cell death/survival in stroke," Annals of the New York Academy of Sciences, vol. 1042, no. 1, pp. 203-209, 2005.

[98] S. Ramagiri and R. Taliyan, "Neuroprotective effect of hydroxy safflor yellow A against cerebral ischemiareperfusion injury in rats: putative role of mPTP," Journal of Basic and Clinical Physiology and Pharmacology, vol. 27, no. 1, pp. 1-8, 2016.

[99] J. Sun, D. D. Ren, J. Y. Wan et al., "Desensitizing mitochondrial permeability transition by ERK-cyclophilin D axis contributes to the neuroprotective effect of gallic acid against cerebral ischemia/reperfusion injury," Frontiers in Pharmacology, vol. 8, p. 184, 2017.

[100] S. Li, T. Wang, L. Zhai et al., "Picroside II exerts a neuroprotective effect by inhibiting MPTP permeability and EndoG release after cerebral ischemia/reperfusion injury in rats," Journal of Molecular Neuroscience, vol. 64, no. 1, pp. 144$155,2018$.

[101] S. Li, C. Wu, L. Zhu et al., "By improving regional cortical blood flow, attenuating mitochondrial dysfunction and sequential apoptosis galangin acts as a potential neuroprotective agent after acute ischemic stroke," Molecules, vol. 17, no. 11, pp. 13403-13423, 2012.

[102] T. F. Franke, C. P. Hornik, L. Segev, G. A. Shostak, and C. Sugimoto, "PI3K/Akt and apoptosis: size matters," Oncogene, vol. 22, no. 56, pp. 8983-8998, 2003.
[103] J. Tao, Y. Cui, Y. Duan, N. Zhang, C. Wang, and F. Zhang, "Puerarin attenuates locomotor and cognitive deficits as well as hippocampal neuronal injury through the PI3K/Akt1/GSK-3 $\beta$ signaling pathway in anin vivomodel of cerebral ischemia," Oncotarget, vol. 8, no. 63, pp. 106283106295, 2017.

[104] C. Wang, Z. Wang, X. Zhang et al., "Protection by silibinin against experimental ischemic stroke: Up-regulated pAkt, pmTOR, HIF- $1 \alpha$ and Bcl-2, down-regulated Bax, NF- $\kappa$ B expression, NF-kappaB expression," Neuroscience Letters. Supplement, vol. 529, no. 1, pp. 45-50, 2012.

[105] P. Wang, Y. F. Guan, H. du, Q. W. Zhai, D. F. Su, and C. Y. Miao, "Induction of autophagy contributes to the neuroprotection of nicotinamide phosphoribosyltransferase in cerebral ischemia," Autophagy, vol. 8, no. 1, pp. 77-87, 2012.

[106] Z. Qi, F. Yan, W. Shi et al., "AKT-related autophagy contributes to the neuroprotective efficacy of hydroxysafflor yellow A against ischemic stroke in rats," Translational Stroke Research, vol. 5, no. 4, pp. 501-509, 2014.

[107] S. M. Jin and R. J. Youle, "PINK1- and Parkin-mediated mitophagy at a glance," Journal of Cell Science, vol. 125, no. 4, pp. 795-799, 2012.

[108] P. Wang, B. Z. Shao, Z. Deng, S. Chen, Z. Yue, and C. Y. Miao, "Autophagy in ischemic stroke," Progress in Neurobiology, vol. 163-164, pp. 98-117, 2018.

[109] S. Yang, H. Wang, Y. Yang et al., "Baicalein administered in the subacute phase ameliorates ischemia-reperfusioninduced brain injury by reducing neuroinflammation and neuronal damage," Biomedicine \& Pharmacotherapy, vol. 117, article 109102, 2019.

[110] H. Hongyun, G. Tao, Z. Pengyue, Y. Liqiang, and D. Yihao, "Puerarin provides a neuroprotection against transient cerebral ischemia by attenuating autophagy at the ischemic penumbra in neurons but not in astrocytes," Neuroscience Letters, vol. 643, pp. 45-51, 2017.

[111] J. Feng, X. Chen, and J. Shen, "Reactive nitrogen species as therapeutic targets for autophagy: implication for ischemic stroke," Expert Opinion on Therapeutic Targets, vol. 21, no. 3, pp. 305-317, 2017.

[112] A. Rami, "Review: autophagy in neurodegeneration: firefighter and/or incendiarist?," Neuropathology and Applied Neurobiology, vol. 35, no. 5, pp. 449-461, 2009.

[113] Y. Yang, K. Gao, Z. Hu et al., “Autophagy upregulation and apoptosis downregulation in DAHP and triptolide treated cerebral ischemia," Mediators of Inflammation, vol. 2015, Article ID 120198, 12 pages, 2015.

[114] Y. Zhang, Y. Zhang, X. F. Jin et al., "The role of astragaloside IV against cerebral ischemia/reperfusion injury: suppression of apoptosis via promotion of P62-LC3-autophagy," Molecules, vol. 24, no. 9, p. 1838, 2019.

[115] T. Lu, Y. Jiang, Z. Zhou et al., "Intranasal ginsenoside Rb1 targets the brain and ameliorates cerebral ischemia/reperfusion injury in rats," Biological \& Pharmaceutical Bulletin, vol. 34, no. 8, pp. 1319-1324, 2011.

[116] S. Cao, S. Shrestha, J. Li et al., "Melatonin-mediated mitophagy protects against early brain injury after subarachnoid hemorrhage through inhibition of NLRP3 inflammasome activation," Scientific Reports, vol. 7, no. 1, article 2417, 2017.

[117] N. K. Zenkov, A. V. Chechushkov, P. M. Kozhin, N. V. Kandalintseva, G. G. Martinovich, and E. B. Menshchikova, 
"Plant phenols and autophagy," Biochemistry (Moscow), vol. 81, no. 4, pp. 297-314, 2016.

[118] J. Houlton, N. Abumaria, S. F. R. Hinkley, and A. N. Clarkson, "Therapeutic potential of neurotrophins for repair after brain injury: a helping hand from biomaterials," Frontiers in Neuroscience, vol. 13, p. 790, 2019.

[119] H. Liu, L. Zhong, Y. Zhang, X. Liu, and J. Li, "Rutin attenuates cerebral ischemia-reperfusion injury in ovariectomized rats via estrogen-receptor-mediated BDNF-TrkB and NGFTrkA signaling," Biochemistry and Cell Biology, vol. 96, no. 5, pp. 672-681, 2018.

[120] L. Pan, Y. Zhou, X. F. Li, Q. J. Wan, and L. H. Yu, "Preventive treatment of astaxanthin provides neuroprotection through suppression of reactive oxygen species and activation of antioxidant defense pathway after stroke in rats," Brain Research Bulletin, vol. 130, pp. 211-220, 2017.

[121] D. Wu, "Neuroprotection in experimental stroke with targeted neurotrophins," NeuroRx, vol. 2, no. 1, pp. 120-128, 2005.

[122] J. Kim, D. Y. W. Fann, R. C. S. Seet, D. G. Jo, M. P. Mattson, and T. V. Arumugam, "Phytochemicals in ischemic stroke," Neuromolecular Medicine, vol. 18, no. 3, pp. 283-305, 2016.

[123] H. S. Chen, S. H. Qi, and J. G. Shen, "One-compoundmulti-target: combination prospect of natural compounds with thrombolytic therapy in acute ischemic stroke," Current Neuropharmacology, vol. 15, no. 1, pp. 134-156, 2017.

[124] C. Gutierrez-Merino, C. Lopez-Sanchez, R. Lagoa, K. A. Samhan-Arias, C. Bueno, and V. Garcia-Martinez, "Neuroprotective actions of flavonoids," Current Medicinal Chemistry, vol. 18, no. 8, pp. 1195-1212, 2011.

[125] F. Tu, Q. Pang, T. Huang, Y. Zhao, M. Liu, and X. Chen, "Apigenin ameliorates post-stroke cognitive deficits in rats through histone acetylation-mediated neurochemical alterations," Medical Science Monitor, vol. 23, pp. 4004-4013, 2017.

[126] M. Cai, Y. Ma, W. Zhang et al., “Apigenin-7-O- $\beta-D-(-6 "-p-$ coumaroyl)-Glucopyranoside treatment elicits neuroprotective effect against experimental ischemic stroke," International Journal of Biological Sciences, vol. 12, no. 1, pp. 4252, 2016.

[127] Y. Wang, Y. Zhen, X. Wu et al., "Vitexin protects brain against ischemia/reperfusion injury via modulating mitogen-activated protein kinase and apoptosis signaling in mice," Phytomedicine, vol. 22, no. 3, pp. 379-384, 2015.

[128] M. Xu, X. Chen, Y. Gu et al., "Baicalin can scavenge peroxynitrite and ameliorate endogenous peroxynitrite- mediated neurotoxicity in cerebral ischemia-reperfusion injury," Journal of Ethnopharmacology, vol. 150, no. 1, pp. 116-124, 2013.

[129] X. Xue, X. J. Qu, Y. Yang et al., "Baicalin attenuates focal cerebral ischemic reperfusion injury through inhibition of nuclear factor $\kappa \mathrm{B}$ p65 activation," Biochemical and Biophysical Research Communications, vol. 403, no. 3-4, pp. 398404, 2010.

[130] S. Li, X. Sun, L. Xu et al., "Baicalin attenuates in vivo and in vitro hyperglycemia-exacerbated ischemia/reperfusion injury by regulating mitochondrial function in a manner dependent on AMPK," European Journal of Pharmacology, vol. 815, pp. 118-126, 2017.

[131] P. Wang, Y. Cao, J. Yu et al., "Baicalin alleviates ischemiainduced memory impairment by inhibiting the phosphoryla- tion of CaMKII in hippocampus," Brain Research, vol. 1642, pp. 95-103, 2016.

[132] A. Sarkaki, Y. Farbood, S. M. T. Mansouri et al., "Chrysin prevents cognitive and hippocampal long-term potentiation deficits and inflammation in rat with cerebral hypoperfusion and reperfusion injury," Life Sciences, vol. 226, pp. 202-209, 2019.

[133] M. K. Shooshtari, A. Sarkaki, S. M. T. Mansouri et al., "Protective effects of chrysin against memory impairment, cerebral hyperemia and oxidative stress after cerebral hypoperfusion and reperfusion in rats," Metabolic Brain Disease, vol. 35, no. 2, pp. 401-412, 2020.

[134] X. Liu, X. Zhang, J. Zhang et al., "Diosmin protects against cerebral ischemia/reperfusion injury through activating JAK2/STAT3 signal pathway in mice," Neuroscience, vol. 268, pp. 318-327, 2014.

[135] B. Xu, X. He, Y. Sui et al., "Ginkgetin aglycone attenuates neuroinflammation and neuronal injury in the rats with ischemic stroke by modulating STAT3/JAK2/SIRT1," Folia Neuropathologica, vol. 57, no. 1, pp. 16-23, 2019.

[136] P. An, J. Xie, S. Qiu et al., "Hispidulin exhibits neuroprotective activities against cerebral ischemia reperfusion injury through suppressing NLRP3-mediated pyroptosis," Life Sciences, vol. 232, p. 116599, 2019.

[137] H. Qiao, L. Dong, X. Zhang et al., "Protective effect of luteolin in experimental ischemic stroke: upregulated SOD1, CAT, Bcl-2 and claudin-5, down-regulated MDA and Bax expression," Neurochemical Research, vol. 37, no. 9, pp. 20142024, 2012.

[138] G. Zhao, S. Y. Zang, Z. H. Jiang et al., "Postischemic administration of liposome-encapsulated luteolin prevents against ischemia-reperfusion injury in a rat middle cerebral artery occlusion model," The Journal of Nutritional Biochemistry, vol. 22, no. 10, pp. 929-936, 2011.

[139] Q. Li, Z. Tian, M. Wang et al., "Luteoloside attenuates neuroinflammation in focal cerebral ischemia in rats via regulation of the PPAR $\gamma / \mathrm{Nrf} 2 / \mathrm{NF}-\kappa \mathrm{B}$ signaling pathway," International Immunopharmacology, vol. 66, pp. 309-316, 2019.

[140] X. Wang, F. An, S. Wang, Z. An, and S. Wang, "Orientin attenuates cerebral ischemia/reperfusion injury in rat model through the AQP- 4 and TLR4/NF- $\kappa \mathrm{B} / \mathrm{TNF}-\alpha$ signaling pathway," Journal of Stroke and Cerebrovascular Diseases, vol. 26, no. 10, pp. 2199-2214, 2017.

[141] Y. Zheng, J. Bu, L. Yu, J. Chen, and H. Liu, "Nobiletin improves propofol-induced neuroprotection via regulating Akt/mTOR and TLR 4/NF- $\kappa$ B signaling in ischemic brain injury in rats," Biomedicine \& Pharmacotherapy, vol. 91, pp. 494-503, 2017.

[142] N. Yasuda, T. Ishii, D. Oyama et al., "Neuroprotective effect of nobiletin on cerebral ischemia-reperfusion injury in transient middle cerebral artery-occluded rats," Brain Research, vol. 1559, pp. 46-54, 2014.

[143] L. Zhang, H. Zhao, X. Zhang et al., "Nobiletin protects against cerebral ischemia via activating the $\mathrm{p}$-Akt, $\mathrm{p}$-CREB, BDNF and Bcl-2 pathway and ameliorating BBB permeability in rat," Brain Research Bulletin, vol. 96, pp. 45-53, 2013.

[144] Y. Yuan, H. Zha, P. Rangarajan, E. A. Ling, and C. Wu, “Antiinflammatory effects of edaravone and scutellarin in activated microglia in experimentally induced ischemia injury in rats and in BV-2 microglia," BMC Neuroscience, vol. 15, no. 1, p. $125,2014$. 
[145] H. Guo, L. M. Hu, S. X. Wang et al., "Neuroprotective effects of scutellarin against hypoxic-ischemic-induced cerebral injury via augmentation of antioxidant defense capacity," The Chinese Journal of Physiology, vol. 54, no. 6, pp. 399405, 2011.

[146] L. Chai, H. Guo, H. Li et al., "Scutellarin and caffeic acid ester fraction, active components of Dengzhanxixin injection, upregulate neurotrophins synthesis and release in hypoxia/reoxygenation rat astrocytes," Journal of Ethnopharmacology, vol. 150, no. 1, pp. 100-107, 2013.

[147] W. Wang, X. Ma, J. Han et al., "Neuroprotective effect of scutellarin on ischemic cerebral injury by down-regulating the expression of angiotensin-converting enzyme and AT1 receptor," PLoS One, vol. 11, no. 1, article e0146197, 2016.

[148] W. L. Jiang, Y. Xu, S. P. Zhang, H. B. Zhu, and J. Hou, “Tricin 7-glucoside protects against experimental cerebral ischemia by reduction of NF- $\kappa \mathrm{B}$ and HMGB1 expression," European Journal of Pharmaceutical Sciences, vol. 45, no. 1-2, pp. 50$57,2012$.

[149] H. Huang, Y. Zhang, R. Yang, and X. Tang, "Determination of baicalin in rat cerebrospinal fluid and blood using microdialysis coupled with ultra-performance liquid chromatography-tandem mass spectrometry," Journal of Chromatography. B, Analytical Technologies in the Biomedical and Life Sciences, vol. 874, no. 1-2, pp. 77-83, 2008.

[150] W. Liang, X. Huang, and W. Chen, "The effects of baicalin and baicalein on cerebral ischemia: a review," Aging and Disease, vol. 8, no. 6, pp. 850-867, 2017.

[151] S. Wang, H. Wang, H. Guo, L. Kang, X. Gao, and L. Hu, "Neuroprotection of scutellarin is mediated by inhibition of microglial inflammatory activation," Neuroscience, vol. 185, pp. 150-160, 2011.

[152] E. . O. Ferreira, M. Y. S. D. Fernandes, N. M. R. . Lima et al., "Neuroinflammatory response to experimental stroke is inhibited by eriodictyol," Behavioural Brain Research, vol. 312, pp. 321-332, 2016.

[153] X. Jing, D. Ren, X. Wei et al., "Eriodictyol-7-O-glucoside activates Nrf2 and protects against cerebral ischemic injury," Toxicology and Applied Pharmacology, vol. 273, no. 3, pp. 672-679, 2013.

[154] A. Kumar, S. Lalitha, and J. Mishra, "Possible nitric oxide mechanism in the protective effect of hesperidin against pentylenetetrazole (PTZ)-induced kindling and associated cognitive dysfunction in mice," Epilepsy \& Behavior, vol. 29, no. 1, pp. 103-111, 2013.

[155] S. S. Raza, M. M. Khan, A. Ahmad et al., "Neuroprotective effect of naringenin is mediated through suppression of NF$\kappa \mathrm{B}$ signaling pathway in experimental stroke," Neuroscience, vol. 230, pp. 157-171, 2013.

[156] J. Feng, X. Chen, S. Lu et al., "Naringin attenuates cerebral ischemia-reperfusion injury through inhibiting peroxynitritemediated mitophagy activation," Molecular Neurobiology, vol. 55, no. 12, pp. 9029-9042, 2018.

[157] J. J. Wang and P. Cui, "Neohesperidin attenuates cerebral ischemia-reperfusion injury via inhibiting the apoptotic pathway and activating the Akt/Nrf2/HO-1 pathway," Journal of Asian Natural Products Research, vol. 15, no. 9, pp. 1023-1037, 2013.

[158] G. Zhao, W. Zhang, L. Li, S. Wu, and G. Du, "Pinocembrin protects the brain against ischemia-reperfusion injury and reverses the autophagy dysfunction in the penumbra area," Molecules, vol. 19, no. 10, pp. 15786-15798, 2014.
[159] S. B. Wang, X. B. Pang, M. Gao, L. H. Fang, and G. H. Du, "Pinocembrin protects rats against cerebral ischemic damage through soluble epoxide hydrolase and epoxyeicosatrienoic acids," Chinese Journal of Natural Medicines, vol. 11, no. 3, pp. 207-213, 2013.

[160] F. Meng, Y. Wang, R. Liu, M. Gao, and G. Du, "Pinocembrin alleviates memory impairment in transient global cerebral ischemic rats," Experimental and Therapeutic Medicine, vol. 8, no. 4, pp. 1285-1290, 2014.

[161] X. Lan, W. Wang, Q. Li, and J. Wang, "The natural flavonoid pinocembrin: molecular targets and potential therapeutic applications," Molecular Neurobiology, vol. 53, no. 3, pp. 1794-1801, 2016.

[162] X. Shen, Y. Liu, X. Luo, and Z. Yang, “Advances in biosynthesis, pharmacology, and pharmacokinetics of pinocembrin, a promising natural small-molecule drug," Molecules, vol. 24, no. 12, p. 2323, 2019.

[163] Z. A. Shah, R. C. Li, A. S. Ahmad et al., "The flavanol (-)-epicatechin prevents stroke damage through the Nrf2/HO1 pathway," Journal of Cerebral Blood Flow \& Metabolism, vol. 30, no. 12, pp. 1951-1961, 2010.

[164] X. Wang and Y. You, "Epigallocatechin gallate extends therapeutic window of recombinant tissue plasminogen activator treatment for brain ischemic stroke: a randomized doubleblind and placebo-controlled trial," Clinical Neuropharmacology, vol. 40, no. 1, pp. 24-28, 2017.

[165] Y. P. You, "Epigallocatechin gallate extends the therapeutic window of recombinant tissue plasminogen activator treatment in ischemic rats," Journal of Stroke and Cerebrovascular Diseases, vol. 25, no. 4, pp. 990-997, 2016.

[166] J. Han, M. Wang, X. Jing, H. Shi, M. Ren, and H. Lou, "(-)-Epigallocatechin gallate protects against cerebral ischemia-induced oxidative stress via Nrf2/ARE signaling," Neurochemical Research, vol. 39, no. 7, pp. 1292-1299, 2014.

[167] F. Zhang, N. Li, L. Jiang, L. Chen, and M. Huang, "Neuroprotective effects of (-)-epigallocatechin-3-gallate against focal cerebral ischemia/reperfusion injury in rats through attenuation of inflammation," Neurochemical Research, vol. 40, no. 8, pp. 1691-1698, 2015.

[168] W. Nan, X. Zhonghang, C. Keyan, L. Tongtong, G. Wanshu, and X. Zhongxin, "Epigallocatechin-3-gallate reduces neuronal apoptosis in rats after middle cerebral artery occlusion injury via PI3K/Akt/eNOS signaling pathway," BioMed Research International, vol. 2018, Article ID 6473580, 9 pages, 2018.

[169] C. Yao, J. Zhang, G. Liu, F. Chen, and Y. Lin, "Neuroprotection by (-)-epigallocatechin-3-gallate in a rat model of stroke is mediated through inhibition of endoplasmic reticulum stress," Molecular Medicine Reports, vol. 9, no. 1, pp. 69-72, 2014.

[170] J. W. Park, J. S. Hong, K. S. Lee, H. Y. Kim, J. J. Lee, and S. R. Lee, "Green tea polyphenol (-)-epigallocatechin gallate reduces matrix metalloproteinase- 9 activity following transient focal cerebral ischemia," Journal of Nutritional Biochemistry, vol. 21, no. 11, pp. 1038-1044, 2010.

[171] S. Wu, Y. Yue, J. Li et al., "Procyanidin B2 attenuates neurological deficits and blood-brain barrier disruption in a rat model of cerebral ischemia," Molecular Nutrition \& Food Research, vol. 59, no. 10, pp. 1930-1941, 2015.

[172] S. Jin, M. Park, and J. H. Song, “(-)-Epigallocatechin-3-gallate inhibits voltage-gated proton currents in BV2 microglial 
cells," European Journal of Pharmacology, vol. 698, no. 1-3, pp. 154-160, 2013.

[173] Q. Bai, Z. Lyu, X. Yang, Z. Pan, J. Lou, and T. Dong, "Epigallocatechin-3-gallate promotes angiogenesis via upregulation of Nfr2 signaling pathway in a mouse model of ischemic stroke," Behavioural Brain Research, vol. 321, pp. 79-86, 2017.

[174] B. B. Wei, M. Y. Liu, X. Zhong, W. F. Yao, and M. J. Wei, "Increased BBB permeability contributes to EGCG-caused cognitive function improvement in natural aging rats: pharmacokinetic and distribution analyses," Acta Pharmacologica Sinica, vol. 40, no. 11, pp. 1490-1500, 2019.

[175] C. Wu, J. Chen, C. Chen et al., "Wnt/ $\beta$-catenin coupled with HIF-1 $\alpha$ /VEGF signaling pathways involved in galangin neurovascular unit protection from focal cerebral ischemia," Scientific Reports, vol. 5, no. 1, article 16151, 2015.

[176] X. Wang, J. Li, L. Qian et al., "Icariin promotes histone acetylation and attenuates post-stroke cognitive impairment in the central cholinergic circuits of mice," Neuroscience, vol. 236, pp. 281-288, 2013.

[177] H. R. Zhu, Z. Y. Wang, X. L. Zhu, X. X. Wu, E. G. Li, and $\mathrm{Y}$. $\mathrm{Xu}$, "Icariin protects against brain injury by enhancing SIRT1-dependent PGC-1 $\alpha$ Expression in experimental stroke," Neuropharmacology, vol. 59, no. 1-2, pp. 70-76, 2010.

[178] L. Yu, C. Chen, L. F. Wang et al., "Neuroprotective effect of kaempferol glycosides against brain injury and neuroinflammation by inhibiting the activation of NF- $\kappa \mathrm{B}$ and STAT3 in transient focal stroke," PLoS One, vol. 8, no. 2, article e55839, 2013.

[179] S. Wang, H. Xu, Y. Xin et al., "Neuroprotective effects of Kaempferide-7-O-(4"-O-acetylrhamnosyl)-3-O-rutinoside on cerebral ischemia- reperfusion injury in rats," European Journal of Pharmacology, vol. 788, pp. 335-342, 2016.

[180] D. J. Park, J. B. Kang, M. A. Shan, and P. O. Koh, "Quercetin alleviates the injury-induced decrease of protein phosphatase $2 \mathrm{~A}$ subunit $\mathrm{B}$ in cerebral ischemic animal model and glutamate-exposed HT22 cells," Journal of Veterinary Medical Science, vol. 81, no. 7, pp. 1047-1054, 2019.

[181] D. J. Park, F. A. Shah, and P. O. Koh, "Quercetin attenuates neuronal cells damage in a middle cerebral artery occlusion animal model," Journal of Veterinary Medical Science, vol. 80, no. 4, pp. 676-683, 2018.

[182] F. A. Shah, D. J. Park, and P. O. Koh, "Identification of proteins differentially expressed by quercetin treatment in a middle cerebral artery occlusion model: a proteomics approach," Neurochemical Research, vol. 43, no. 8, pp. 1608-1623, 2018.

[183] J. W. Jang, J. K. Lee, H. Hur, T. W. Kim, S. P. Joo, and M. S. Piao, "Rutin improves functional outcome via reducing the elevated matrix metalloproteinase-9 level in a photothrombotic focal ischemic model of rats," Journal of the Neurological Sciences, vol. 339, no. 1-2, pp. 75-80, 2014.

[184] C. P. Wang, Y. W. Shi, M. Tang et al., "Isoquercetin ameliorates cerebral impairment in focal ischemia through anti-oxidative, anti-inflammatory, and anti-apoptotic effects in primary culture of rat hippocampal neurons and hippocampal CA1 region of rats," Molecular Neurobiology, vol. 54, no. 3, pp. 2126-2142, 2017.

[185] J. J. Zhao, J. Q. Song, S. Y. Pan, and K. Wang, "Treatment with isorhamnetin protects the brain against ischemic injury in mice," Neurochemical Research, vol. 41, no. 8, pp. 19391948, 2016.
[186] L. Sun, P. Xu, T. Fu et al., "Myricetin against ischemic cerebral injury in rat middle cerebral artery occlusion model," Molecular Medicine Reports, vol. 17, no. 2, pp. 3274-3280, 2018.

[187] S. Chirumbolo, A. Vella, and G. Bjorklund, "Quercetin might promote autophagy in a middle cerebral artery occlusionmediated ischemia model: comments on Fawad-Ali Shah et al.," Neurochemical Research, vol. 44, no. 2, pp. 297-300, 2019.

[188] R. A. Rifaai, S. A. Mokhemer, E. A. Saber, S. A. A. El-Aleem, and N. F. G. El-Tahawy, "Neuroprotective effect of quercetin nanoparticles: a possible prophylactic and therapeutic role in Alzheimer's disease," Journal of Chemical Neuroanatomy, vol. 107, article 101795, 2020.

[189] A. Annapurna, M. A. Ansari, and P. M. Manjunath, "Partial role of multiple pathways in infarct size limiting effect of quercetin and rutin against cerebral ischemia-reperfusion injury in rats," European Review for Medical and Pharmacological Sciences, vol. 17, no. 4, pp. 491-500, 2013.

[190] C. C. Hsu, T. W. Kuo, W. P. Liu, C. P. Chang, and H. J. Lin, "Calycosin preserves BDNF/TrkB signaling and reduces post-stroke neurological injury after cerebral ischemia by reducing accumulation of hypertrophic and TNF- $\alpha$-Containing microglia in rats," Journal of Neuroimmune Pharmacology, vol. 15, no. 2, pp. 326-339, 2020.

[191] T. Kuo and C. Chang, "Calycosin may improve outcomes of ischemic stroke in rats by inhibiting activated microglia in brain,” FASEB Journal, vol. 29, pp. 834-835, 2015.

[192] S. Fu, Y. Gu, J. Q. Jiang et al., "Calycosin-7-O- $\beta$-d-glucoside regulates nitric oxide /caveolin-1/matrix metalloproteinases pathway and protects blood-brain barrier integrity in experimental cerebral ischemia-reperfusion injury," Journal of Ethnopharmacology, vol. 155, no. 1, pp. 692-701, 2014.

[193] K. Liang, Y. Ye, Y. Wang, J. Zhang, and C. Li, "Formononetin mediates neuroprotection against cerebral ischemia/reperfusion in rats via downregulation of the $\mathrm{Bax} / \mathrm{Bcl}-2$ ratio and upregulation PI3K/Akt signaling pathway," Journal of the Neurological Sciences, vol. 344, no. 1-2, pp. 100104, 2014.

[194] A. B. Aras, M. Guven, T. Akman et al., "Genistein exerts neuroprotective effect on focal cerebral ischemia injury in rats," Inflammation, vol. 38, no. 3, pp. 1311-1321, 2015.

[195] Y. Qian, T. Guan, M. Huang et al., "Neuroprotection by the soy isoflavone, genistein, via inhibition of mitochondriadependent apoptosis pathways and reactive oxygen induced-NF- $\kappa \mathrm{B}$ activation in a cerebral ischemia mouse model," Neurochemistry International, vol. 60 , no. 8 , pp. 759-767, 2012.

[196] B. Cortina, G. Torregrosa, M. Castello-Ruiz et al., "Improvement of the circulatory function partially accounts for the neuroprotective action of the phytoestrogen genistein in experimental ischemic stroke," European Journal of Pharmacology, vol. 708, no. 1-3, pp. 88-94, 2013.

[197] L. Y. Lu, Y. Liu, Y. F. Gong, and X. Y. Zheng, "A preliminary report: genistein attenuates cerebral ischemia injury in ovariectomized rats via regulation of the PI3K-Akt-mTOR pathway," General Physiology and Biophysics, vol. 38, no. 5, pp. 389-397, 2019.

[198] Z. Y. Miao, X. Xia, L. Che, and Y. T. Song, "Genistein attenuates brain damage induced by transient cerebral ischemia through up-regulation of Nrf2 expression in ovariectomized rats," Neurological Research, vol. 40, no. 8, pp. 689-695, 2018. 
[199] S. Wang, H. Wei, M. Cai et al., "Genistein attenuates brain damage induced by transient cerebral ischemia through upregulation of ERK activity in ovariectomized mice," International Journal of Biological Sciences, vol. 10, no. 4, pp. 457465, 2014.

[200] A. B. Aras, M. Guven, T. Akman et al., "Neuroprotective effects of daidzein on focal cerebral ischemia injury in rats," Neural Regeneration Research, vol. 10, no. 1, pp. 146-152, 2015.

[201] Y. Chang, C. Y. Hsieh, Z. A. Peng et al., "Neuroprotective mechanisms of puerarin in middle cerebral artery occlusion-induced brain infarction in rats," Journal of Biomedical Science, vol. 16, no. 1, p. 9, 2009.

[202] X. Liu, Z. Mei, J. Qian, Y. Zeng, and M. Wang, "Puerarin partly counteracts the inflammatory response after cerebral ischemia/reperfusion via activating the cholinergic antiinflammatory pathway," Neural Regeneration Research, vol. 8, no. 34, pp. 3203-3215, 2013.

[203] N. Wang, Y. Zhang, L. Wu et al., "Puerarin protected the brain from cerebral ischemia injury via astrocyte apoptosis inhibition," Neuropharmacology, vol. 79, pp. 282-289, 2014.

[204] D. A. Schreihofer and A. Oppong-Gyebi, "Genistein: mechanisms of action for a pleiotropic neuroprotective agent in stroke," Nutritional Neuroscience, vol. 22, no. 6, pp. 375391, 2019.

[205] J. M. Stout, A. N. Knapp, W. J. Banz, D. G. Wallace, and J. L. Cheatwood, "Subcutaneous daidzein administration enhances recovery of skilled ladder rung walking performance following stroke in rats," Behavioural Brain Research, vol. 256, pp. 428-431, 2013.

[206] Y. Ma, J. C. Sullivan, and D. A. Schreihofer, "Dietary genistein and equol (4', 7 isoflavandiol) reduce oxidative stress and protect rats against focal cerebral ischemia," American Journal of Physiology. Regulatory, Integrative and Comparative Physiology, vol. 299, no. 3, pp. R871-R877, 2010.

[207] A. Kloska, M. Narajczyk, J. Jakóbkiewicz-Banecka et al., "Synthetic genistein derivatives as modulators of glycosaminoglycan storage," Journal of Translational Medicine, vol. 10, no. 1, p. 153, 2012.

[208] B. X. Xiao, L. Feng, F. R. Cao et al., "Pharmacokinetic profiles of the five isoflavonoids from Pueraria lobata roots in the CSF and plasma of rats," Journal of Ethnopharmacology, vol. 184, pp. 22-29, 2016.

[209] M. Yuan, G. Liu, X. Zheng et al., "Effects of puerarin combined with conventional therapy on ischemic stroke," Experimental and Therapeutic Medicine, vol. 14, no. 4, pp. 29432946, 2017.

[210] Q. H. Zheng, X. L. Li, Z. G. Mei et al., "Efficacy and safety of puerarin injection in curing acute ischemic stroke: a metaanalysis of randomized controlled trials," Medicine, vol. 96, no. 1, article e5803, 2017.

[211] J. Min, S. W. Yu, S. H. Baek et al., "Neuroprotective effect of cyanidin-3-_O_-glucoside anthocyanin in mice with focal cerebral ischemia," Neuroscience Letters, vol. 500, no. 3, pp. 157-161, 2011.

[212] Y. Lv, Y. Qian, L. Fu, X. Chen, H. Zhong, and X. Wei, "Hydroxysafflor yellow A exerts neuroprotective effects in cerebral ischemia reperfusion-injured mice by suppressing the innate immune TLR4-inducing pathway," European Journal of Pharmacology, vol. 769, pp. 324-332, 2015.
[213] L. Chen, Y. Xiang, L. Kong et al., "Hydroxysafflor yellow A protects against cerebral ischemia-reperfusion injury by anti-apoptotic effect through PI3K/Akt/GSK3 $\beta$ pathway in rat," Neurochemical Research, vol. 38, no. 11, pp. 22682275, 2013.

[214] L. Deng, H. Wan, H. Zhou, L. Yu, and Y. He, "Protective effect of hydroxysafflor yellow A alone or in combination with acetylglutamine on cerebral ischemia reperfusion injury in rat: A PET study using ${ }^{18} \mathrm{~F}$-fuorodeoxyglucose," European Journal of Pharmacology, vol. 825, pp. 119-132, 2018.

[215] S. Chen, M. Sun, X. Zhao et al., "Neuroprotection of hydroxysafflor yellow A in experimental cerebral ischemia/reperfusion injury via metabolic inhibition of phenylalanine and mitochondrial biogenesis," Molecular Medicine Reports, vol. 19, no. 4, pp. 3009-3020, 2019.

[216] T. Yen, C. Hsu, W. Lu et al., "Neuroprotective effects of xanthohumol, a prenylated flavonoid from hops (Humulus lupulus), in ischemic stroke of rats," Journal of Agricultural and Food Chemistry, vol. 60, no. 8, pp. 1937-1944, 2012.

[217] M. Wang, Y. J. Li, Y. Ding et al., "Silibinin prevents autophagic cell death upon oxidative stress in cortical neurons and cerebral ischemia-reperfusion injury," Molecular Neurobiology, vol. 53, no. 2, pp. 932-943, 2016.

[218] S. S. Raza, M. M. Khan, M. Ashafaq et al., "Silymarin protects neurons from oxidative stress associated damages in focal cerebral ischemia: a behavioral, biochemical and immunohistological study in Wistar rats," Journal of the Neurological Sciences, vol. 309, no. 1-2, pp. 45-54, 2011.

[219] H. Ao, W. Feng, and C. Peng, "Hydroxysafflor yellow A: a promising therapeutic agent for a broad spectrum of diseases," Evidence-based Complementary and Alternative Medicine, vol. 2018, Article ID 8259280, 17 pages, 2018.

[220] X. Wang, Z. Ma, Z. Fu et al., "Hydroxysafflor yellow A protects neurons from excitotoxic death through inhibition of NMDARs," ASN Neuro, vol. 8, no. 2, article $175909141664234,2016$.

[221] P. He, "Effect of cerebral ischemia/reperfusion injury on hydroxysafflor yellow A penetrating across the blood-brain barrier," Scientia Pharmaceutica, vol. 76, no. 4, pp. 713-723, 2008.

[222] M. Dvorakova and P. Landa, "Anti-inflammatory activity of natural stilbenoids: a review," Pharmacological Research, vol. 124, pp. 126-145, 2017.

[223] C. Pang, L. Cao, F. Wu et al., "The effect of_trans_-resveratrol on post-stroke depression via regulation of hypothalamus-pituitary-adrenal axis," Neuropharmacology, vol. 97, pp. 447-456, 2015.

[224] H. Yang, A. Zhang, Y. Zhang, S. Ma, and C. Wang, "Resveratrol pretreatment protected against cerebral ischemia/reperfusion injury in rats via expansion of T regulatory cells," Journal of Stroke and Cerebrovascular Diseases, vol. 25, no. 8, pp. 1914-1921, 2016.

[225] J. Ren, C. Fan, N. Chen, J. Huang, and Q. Yang, "Resveratrol pretreatment attenuates cerebral ischemic injury by upregulating expression of transcription factor Nrf2 and $\mathrm{HO}-1$ in rats," Neurochemical Research, vol. 36, no. 12, pp. 23522362, 2011.

[226] Z. Li, L. Pang, F. Fang et al., "Resveratrol attenuates brain damage in a rat model of focal cerebral ischemia via upregulation of hippocampal Bcl-2," Brain Research, vol. 1450, pp. 116-124, 2012. 
[227] H. Wei, S. Wang, L. Zhen et al., "Resveratrol attenuates the blood-brain barrier dysfunction by regulation of the MMP9/TIMP-1 balance after cerebral ischemia reperfusion in rats," Journal of Molecular Neuroscience, vol. 55, no. 4, pp. 872-879, 2015.

[228] K. B. Koronowski, N. Khoury, I. Saul et al., "Neuronal SIRT1 (silent information regulator 2 homologue 1) regulates glycolysis and mediates resveratrol-induced ischemic tolerance," Stroke, vol. 48, no. 11, pp. 3117-3125, 2017.

[229] D. Wan, Y. Zhou, K. Wang, Y. Hou, R. Hou, and X. Ye, "Resveratrol provides neuroprotection by inhibiting phosphodiesterases and regulating the cAMP/AMPK/SIRT1 pathway after stroke in rats," Brain Research Bulletin, vol. 121, pp. 255-262, 2016.

[230] L. M. Wang, Y. J. Wang, M. Cui et al., “A dietary polyphenol resveratrol acts to provide neuroprotection in recurrent stroke models by regulating AMPK and SIRT1 signaling, thereby reducing energy requirements during ischemia," The European Journal of Neuroscience, vol. 37, no. 10, pp. 1669-1681, 2013.

[231] K. B. Koronowski, K. R. Dave, I. Saul et al., "Resveratrol preconditioning induces a novel extended window of ischemic tolerance in the mouse brain," Stroke, vol. 46, no. 8, pp. 2293-2298, 2015.

[232] N. Khoury, J. Xu, S. D. Stegelmann et al., "Resveratrol preconditioning induces genomic and metabolic adaptations within the long-term window of cerebral ischemic tolerance leading to bioenergetic efficiency," Molecular Neurobiology, vol. 56, no. 6, pp. 4549-4565, 2019.

[233] P. Yu, L. Wang, F. Tang et al., "Resveratrol pretreatment decreases ischemic injury and improves neurological function via sonic hedgehog signaling after stroke in rats," Molecular Neurobiology, vol. 54, no. 1, pp. 212-226, 2017.

[234] M. C. Saleh, B. J. Connell, and T. M. Saleh, "Resveratrol preconditioning induces cellular stress proteins and is mediated via NMDA and estrogen receptors," Neuroscience, vol. 166, no. 2, pp. 445-454, 2010.

[235] Z. Dou, X. Rong, E. Zhao, L. Zhang, and Y. Lv, "Neuroprotection of resveratrol against focal cerebral ischemia/reperfusion injury in mice through a mechanism targeting gut-brain axis," Cellular and Molecular Neurobiology, vol. 39, no. 6, pp. 883-898, 2019.

[236] Y. Gao, T. Chen, X. Lei et al., "Neuroprotective effects of polydatin against mitochondrial-dependent apoptosis in the rat cerebral cortex following ischemia/reperfusion injury," Molecular Medicine Reports, vol. 14, no. 6, pp. 5481-5488, 2016.

[237] H. Ji, X. Zhang, Y. Du, H. Liu, S. Li, and L. Li, "Polydatin modulates inflammation by decreasing NF- $\kappa$ B activation and oxidative stress by increasing Gli1, Ptch1, SOD1 expression and ameliorates blood-brain barrier permeability for its neuroprotective effect in pMCAO rat brain," Brain Research Bulletin, vol. 87, no. 1, pp. 50-59, 2012.

[238] W. Yang, X. Chen, J. Pan et al., "Malibatol A protects against brain injury through reversing mitochondrial dysfunction in experimental stroke," Neurochemistry International, vol. 80, pp. 33-40, 2015.

[239] S. A. Andrabi, M. G. Spina, P. Lorenz, U. Ebmeyer, G. Wolf, and T. F. Horn, "Oxyresveratrol (trans-2,3',4,5' -tetrahydroxystilbene) is neuroprotective and inhibits the apoptotic cell death in transient cerebral ischemia," Brain Research, vol. 1017, no. 1-2, pp. 98-107, 2004.
[240] C. P. Wang, L. Z. Zhang, G. C. Li et al., "Mulberroside A protects against ischemic impairment in primary culture of rat cortical neurons after oxygen-glucose deprivation followed by reperfusion," Journal of Neuroscience Research, vol. 92, no. 7, pp. 944-954, 2014.

[241] Y. Zhou, X. M. Zhang, A. Ma et al., "Orally administrated pterostilbene attenuates acute cerebral ischemia- reperfusion injury in a dose- and time-dependent manner in mice," Pharmacology, Biochemistry, and Behavior, vol. 135, pp. 199-209, 2015.

[242] Y. Mu, Z. Xu, X. Zhou et al., "2,3,5,4'-Tetrahydroxystilbene$2-O-\beta$-D-Glucoside attenuates ischemia/reperfusioninduced brain injury in rats by promoting angiogenesis," Planta Medica, vol. 83, no. 8, pp. 676-683, 2017.

[243] N. Nakamichi, R. Fukumori, T. Takarada et al., "Preferential inhibition by antidiarrheic 2-methoxy-4-methylphenol of $\mathrm{Ca}(2+)$ influx across acquired $\mathrm{N}$-methyl-D-aspartate receptor channels composed of NR1/NR2B subunit assembly," Journal of Neuroscience Research, vol. 88, no. 11, pp. 24832493, 2010.

[244] C. J. Xie, A. P. Gu, J. Cai, Y. Wu, and R. C. Chen, "Curcumin protects neural cells against ischemic injury in N2a cells and mouse brain with ischemic stroke," Brain and Behavior: A Cognitive Neuroscience Perspective, vol. 8, no. 2, article e00921, 2018.

[245] S. Altinay, M. Cabalar, C. Isler et al., "Is chronic curcumin supplementation neuroprotective against ischemia for antioxidant activity, neurological deficit, or neuronal apoptosis in an experimental stroke model?," Turkish Neurosurgery, vol. 27, no. 4, pp. 537-545, 2017.

[246] Y. Miao, S. Zhao, Y. Gao et al., "Curcumin pretreatment attenuates inflammation and mitochondrial dysfunction in experimental stroke: the possible role of Sirt1 signaling," Brain Research Bulletin, vol. 121, pp. 9-15, 2016.

[247] W. Li, N. C. Suwanwela, and S. Patumraj, "Curcumin by down-regulating NF-kB and elevating Nrf2, reduces brain edema and neurological dysfunction after cerebral I/R," Microvascular Research, vol. 106, pp. 117-127, 2016.

[248] J. Wu, Q. Li, X. Wang et al., "Neuroprotection by curcumin in ischemic brain injury involves the Akt/Nrf2 pathway," PLoS One, vol. 8, no. 3, article e59843, 2013.

[249] M. Xia, Z. Ye, Y. Shi, L. Zhou, and Y. Hua, "Curcumin improves diabetes mellitus associated cerebral infarction by increasing the expression of GLUT1 and GLUT3," Molecular Medicine Reports, vol. 17, no. 1, pp. 1963-1969, 2018.

[250] M. Ceprian, L. Jimenez-Sanchez, C. Vargas, L. Barata, W. Hind, and J. Martinez-Orgado, "Cannabidiol reduces brain damage and improves functional recovery in a neonatal rat model of arterial ischemic stroke," Neuropharmacology, vol. 116, pp. 151-159, 2017.

[251] S. Khaksar and M. R. Bigdeli, "Intra-cerebral cannabidiol infusion-induced neuroprotection is partly associated with the TNF- $\alpha /$ TNFR1/NF-кB pathway in transient focal cerebral ischaemia," Brain Injury, vol. 31, no. 13-14, pp. 19321943, 2017.

[252] S. Khaksar and M. R. Bigdeli, "Anti-excitotoxic effects of cannabidiol are partly mediated by enhancement of NCX2 and NCX3 expression in animal model of cerebral ischemia," European Journal of Pharmacology, vol. 794, pp. 270-279, 2017.

[253] J. Calahorra, J. Shenk, V. H. Wielenga et al., "Hydroxytyrosol, the major phenolic compound of olive oil, as an acute 
therapeutic strategy after ischemic stroke," Nutrients, vol. 11, no. 10, p. 2430, 2019.

[254] D. Xia, Z. Zhang, and Y. Zhao, "Acteoside attenuates oxidative stress and neuronal apoptosis in rats with focal cerebral ischemia-reperfusion injury," Biological \& Pharmaceutical Bulletin, vol. 41, no. 11, pp. 1645-1651, 2018.

[255] J. Ha Park, K. Y. Yoo, I. Hye Kim et al., "Hydroquinone strongly alleviates focal ischemic brain injury via blockage of blood-brain barrier disruption in rats," Toxicological Sciences, vol. 154, no. 2, pp. 430-441, 2016.

[256] K. Gao, M. Liu, Y. Ding et al., "A phenolic amide (LyA) isolated from the fruits oflycium barbarumprotects against cerebral ischemia-reperfusion injury via $\mathrm{PKC} \varepsilon / \mathrm{Nrf} 2 / \mathrm{HO}-1$ pathway," Aging, vol. 11, no. 24, pp. 12361-12374, 2019.

[257] H. Yu, P. Liu, H. Tang et al., "Oleuropein, a natural extract from plants, offers neuroprotection in focal cerebral ischemia/reperfusion injury in mice," European Journal of Pharmacology, vol. 775, pp. 113-119, 2016.

[258] W. Zhang, X. Liu, and Q. Li, "Protective effects of oleuropein against cerebral ischemia/reperfusion by inhibiting neuronal apoptosis," Medical Science Monitor, vol. 24, pp. 6587-6598, 2018.

[259] W. Lai, X. Xie, X. Zhang et al., "Inhibition of complement drives increase in early growth response proteins and neuroprotection mediated by salidroside after cerebral ischemia," Inflammation, vol. 41, no. 2, pp. 449-463, 2018.

[260] W. Lai, Z. Zheng, X. Zhang et al., "Salidroside-mediated neuroprotection is associated with induction of early growth response genes (Egrs) across a wide therapeutic window," Neurotoxicity Research, vol. 28, no. 2, pp. 108-121, 2015.

[261] Y. Wei, H. Hong, X. Zhang et al., "Salidroside inhibits inflammation through PI3K/Akt/HIF signaling after focal cerebral ischemia in rats," Inflammation, vol. 40, no. 4, pp. 12971309, 2017.

[262] X. Zhang, Q. Du, Y. Yang et al., "Salidroside alleviates ischemic brain injury in mice with ischemic stroke through regulating BDNK mediated PI3K/Akt pathway," Biochemical Pharmacology, vol. 156, pp. 99-108, 2018.

[263] X. Zhang, W. Lai, X. Ying et al., "Salidroside reduces inflammation and brain injury after permanent middle cerebral artery occlusion in rats by regulating $\mathrm{PI} 3 \mathrm{~K} / \mathrm{PKB} / \mathrm{Nrf} 2 / \mathrm{NF} \kappa \mathrm{B}$ signaling rather than complement C3 Activity," Inflammation, vol. 42, no. 5, pp. 1830-1842, 2019.

[264] W. Zuo, F. Yan, B. Zhang, X. Hu, and D. Mei, "Salidroside improves brain ischemic injury by activating PI3K/Akt pathway and reduces complications induced by delayed tPA treatment," European Journal of Pharmacology, vol. 830, pp. 128138, 2018.

[265] J. Y. Na, K. Song, J. W. Lee, S. Kim, and J. Kwon, "Pretreatment of 6-shogaol attenuates oxidative stress and inflammation in middle cerebral artery occlusion-induced mice," European Journal of Pharmacology, vol. 788, pp. 241-247, 2016.

[266] S. S. Yu, J. Zhao, S. P. Lei, X. M. Lin, L. L. Wang, and Y. Zhao, "4-Hydroxybenzyl alcohol ameliorates cerebral injury in rats by antioxidant action," Neurochemical Research, vol. 36, no. 2, pp. 339-346, 2011.

[267] F. He, X. Duan, R. Dai, Y. Li, and Q. Lin, "Protective effect of 4-methoxy benzyl alcohol on the blood-brain barrier after cerebral ischemia reperfusion injury," Journal of Stroke and Cerebrovascular Diseases, vol. 26, no. 6, pp. 1258-1265, 2017.
[268] T. Y. Chen, S. H. Tai, E. J. Lee et al., "Cinnamophilin offers prolonged neuroprotection against gray and white matter damage and improves functional and electrophysiological outcomes after transient focal cerebral ischemia," Critical Care Medicine, vol. 39, no. 5, pp. 1130-1137, 2011.

[269] Y. Lin, J. C. Zhang, J. Fu et al., "Hyperforin attenuates brain damage induced by transient middle cerebral artery occlusion (MCAO) in rats via inhibition of TRPC6 channels degradation," Journal of Cerebral Blood Flow and Metabolism, vol. 33, no. 2, pp. 253-262, 2013.

[270] L. Yaidikar, B. Byna, and S. R. Thakur, "Neuroprotective Effect of Punicalagin against Cerebral Ischemia Reperfusioninduced Oxidative Brain Injury in Rats," Journal of Stroke and Cerebrovascular Diseases, vol. 23, no. 10, pp. 28692878, 2014.

[271] L. Yaidikar and S. Thakur, "Punicalagin attenuated cerebral ischemia-reperfusion insult via inhibition of proinflammatory cytokines, up-regulation of $\mathrm{Bcl}-2$, down-regulation of Bax, and caspase-3," Molecular and Cellular Biochemistry, vol. 402, no. 1-2, pp. 141-148, 2015.

[272] F. D. Pinheiro Fernandes, A. P. Fontenele Menezes, J. C. de Sousa Neves et al., "Caffeic acid protects mice from memory deficits induced by focal cerebral ischemia," Behavioural Pharmacology, vol. 25, no. 7, pp. 637-647, 2014.

[273] J. H. Sung, S. A. Gim, and P. O. Koh, "Ferulic acid attenuates the cerebral ischemic injury-induced decrease in peroxiredoxin-2 and thioredoxin expression," Neuroscience Letters, vol. 566, pp. 88-92, 2014.

[274] J. Sun, Y. Z. Li, Y. H. Ding et al., "Neuroprotective effects of gallic acid against hypoxia/reoxygenation-induced mitochondrial dysfunctions in vitro and cerebral ischemia/reperfusion injury in vivo," Brain Research, vol. 1589, pp. 126139, 2014.

[275] A. A. Fonteles, C. M. de Souza, J. C. de Sousa Neves et al., "Rosmarinic acid prevents against memory deficits in ischemic mice," Behavioural Brain Research, vol. 297, pp. 91103, 2016.

[276] H. Luan, Z. Kan, Y. Xu, C. Lv, and W. Jiang, "Rosmarinic acid protects against experimental diabetes with cerebral ischemia: relation to inflammation response," Journal of Neuroinflammation, vol. 10, no. 1, p. 810, 2013.

[277] S. Q. Feng, N. Aa, J. L. Geng et al., "Pharmacokinetic and metabolomic analyses of the neuroprotective effects of salvianolic acid A in a rat ischemic stroke model," Acta Pharmacologica Sinica, vol. 38, no. 11, pp. 1435-1444, 2017.

[278] K. Bavarsad, G. E. Barreto, M. A. Hadjzadeh, and A. Sahebkar, "Protective effects of curcumin against ischemia-reperfusion injury in the nervous system," Molecular Neurobiology, vol. 56, no. 2, pp. 1391-1404, 2019.

[279] Y. Li, J. Li, S. Li et al., "Curcumin attenuates glutamate neurotoxicity in the hippocampus by suppression of ER stressassociated TXNIP/NLRP3 inflammasome activation in a manner dependent on AMPK," Toxicology and Applied Pharmacology, vol. 286, no. 1, pp. 53-63, 2015.

[280] P. Wicha, J. Tocharus, A. Janyou et al., "Hexahydrocurcumin protects against cerebral ischemia/reperfusion injury, attenuates inflammation, and improves antioxidant defenses in a rat stroke model," PLoS One, vol. 12, no. 12, article e0189211, 2017.

[281] J. Fernandez-Ruiz, M. A. Moro, and J. Martinez-Orgado, "Cannabinoids in neurodegenerative disorders and 
stroke/brain trauma: from preclinical models to clinical applications," Neurotherapeutics, vol. 12, no. 4, pp. 793-806, 2015.

[282] T. J. England, W. H. Hind, N. A. Rasid, and S. E. O'Sullivan, "Cannabinoids in experimental stroke: a systematic review and meta-analysis," Journal of Cerebral Blood Flow and Metabolism, vol. 35, no. 3, pp. 348-358, 2015.

[283] F. Calapai, L. Cardia, E. E. Sorbara et al., "Cannabinoids, blood-brain barrier, and brain disposition," Pharmaceutics, vol. 12 , no. 3 , p. $265,2020$.

[284] Z. Zhong, J. Han, J. Zhang, Q. Xiao, J. Hu, and L. Chen, "Pharmacological activities, mechanisms of action, and safety of salidroside in the central nervous system," Drug Design, Development and Therapy, vol. Volume 12, pp. 1479-1489, 2018.

[285] F. Fan, L. Yang, R. Li et al., "Salidroside as a potential neuroprotective agent for ischemic stroke: a review of sources, pharmacokinetics, mechanism and safety," Biomedicine \& Pharmacotherapy, vol. 129, article 110458, 2020.

[286] S. Hou, M. M. Zhao, P. P. Shen, X. P. Liu, Y. Sun, and J. C. Feng, "Neuroprotective effect of salvianolic acids against cerebral ischemia/reperfusion injury," International Journal of Molecular Sciences, vol. 17, no. 7, p. 1190, 2016.

[287] P. Zhuang, Y. Wan, S. Geng et al., "Salvianolic acids for injection (SAFI) suppresses inflammatory responses in activated microglia to attenuate brain damage in focal cerebral ischemia," Journal of Ethnopharmacology, vol. 198, pp. 194-204, 2017.

[288] Y. Zhang, X. Zhang, L. Cui et al., "Salvianolic acids for injection (SAFI) promotes functional recovery and neurogenesis via sonic hedgehog pathway after stroke in mice," Neurochemistry International, vol. 110, pp. 38-48, 2017.

[289] T. Dong, N. Chen, X. Ma et al., “The protective roles of_L-_ borneolum,_D__ borneolum and synthetic borneol in cerebral ischaemia via modulation of the neurovascular unit," Biomedicine \& Pharmacotherapy, vol. 102, pp. 874-883, 2018.

[290] H. Y. Wu, Y. Tang, L. Y. Gao et al., "The synergetic effect of edaravone and borneol in the rat model of ischemic stroke," European Journal of Pharmacology, vol. 740, pp. 522-531, 2014.

[291] X. Guan, X. Li, X. Yang et al., "The neuroprotective effects of carvacrol on ischemia/reperfusion-induced hippocampal neuronal impairment by ferroptosis mitigation," Life Sciences, vol. 235, article 116795, 2019.

[292] Z. Li, C. Hua, X. Pan, X. Fu, and W. Wu, "Carvacrol exerts neuroprotective effects via suppression of the inflammatory response in middle cerebral artery occlusion rats," Inflammation, vol. 39, no. 4, pp. 1566-1572, 2016.

[293] H. Yu, Z. L. Zhang, J. Chen et al., "Carvacrol, a food-additive, provides neuroprotection on focal cerebral ischemia/reperfusion injury in mice," PLoS One, vol. 7, no. 3, article e33584, 2012.

[294] W. Dong, Y. Xian, W. Yuan et al., "Catalpol stimulates VEGF production via the JAK2/STAT3 pathway to improve angiogenesis in rats' stroke model," Journal of Ethnopharmacology, vol. 191, pp. 169-179, 2016.

[295] Y. R. Liu, R. Y. Lei, C. E. Wang et al., "Effects of catalpol on ATPase and amino acids in gerbils with cerebral ischemia/reperfusion injury," Neurological Sciences, vol. 35, no. 8, pp. 1229-1233, 2014.
[296] W. L. Jiang, S. P. Zhang, H. B. Zhu, Jian-Hou, and J. W. Tian, "Cornin ameliorates cerebral infarction in rats by antioxidant action and stabilization of mitochondrial function," Phytotherapy Research, vol. 24, no. 4, pp. 547-552, 2010.

[297] B. Zhao, L. K. Sun, X. Jiang et al., "Genipin protects against cerebral ischemia-reperfusion injury by regulating the UCP2-SIRT3 signaling pathway," European Journal of Pharmacology, vol. 845, pp. 56-64, 2019.

[298] A. M. Sabogal-Guaqueta, R. Posada-Duque, N. C. Cortes, J. D. Arias-Londono, and G. P. Cardona-Gomez, "Changes in the hippocampal and peripheral phospholipid profiles are associated with neurodegeneration hallmarks in a longterm global cerebral ischemia model: attenuation by linalool," Neuropharmacology, vol. 135, pp. 555-571, 2018.

[299] O. Ciftci, M. N. Oztanir, and A. Cetin, "Neuroprotective effects of $\beta$-Myrcene following global cerebral ischemia/reperfusion-mediated oxidative and neuronal damage in a C57BL/J6 mouse," Neurochemical Research, vol. 39, no. 9, pp. 1717-1723, 2014.

[300] R. B. Guo, G. F. Wang, A. P. Zhao, J. Gu, X. L. Sun, and G. Hu, "Paeoniflorin protects against ischemia-induced brain damages in rats via inhibiting MAPKs/NF- $\kappa$ B-Mediated inflammatory responses," PLoS One, vol. 7, no. 11, article e49701, 2012.

[301] Y. Zhang, H. Li, M. Huang et al., "Paeoniflorin, a monoterpene glycoside, protects the brain from cerebral ischemic injury via inhibition of apoptosis," The American Journal of Chinese Medicine, vol. 43, no. 3, pp. 543-557, 2015.

[302] Y. Zhang, L. Qiao, W. Xu et al., "Paeoniflorin attenuates cerebral ischemia-induced injury by regulating $\mathrm{Ca} 2+/ \mathrm{CaMKII} /-$ CREB signaling pathway," Molecules, vol. 22, no. 3, p. 359, 2017.

[303] L. Xu, Y. Li, Q. Fu, and S. Ma, "Perillaldehyde attenuates cerebral ischemia-reperfusion injury-triggered overexpression of inflammatory cytokines via modulating Akt/JNK pathway in the rat brain cortex," Biochemical and Biophysical Research Communications, vol. 454, no. 1, pp. 65-70, 2014.

[304] R. Tabassum, K. Vaibhav, P. Shrivastava et al., "Perillyl alcohol improves functional and histological outcomes against ischemia-reperfusion injury by attenuation of oxidative stress and repression of COX-2, NOS- 2 and NF- $\kappa$ B in middle cerebral artery occlusion rats," European Journal of Pharmacology, vol. 747, pp. 190-199, 2015.

[305] M. Khoshnazar, M. R. Bigdeli, S. Parvardeh, and R. Pouriran, "Attenuating effect of $\alpha$-pinene on neurobehavioural deficit, oxidative damage and inflammatory response following focal ischaemic stroke in rat," The Journal of Pharmacy and Pharmacology, vol. 71, no. 11, pp. 1725-1733, 2019.

[306] Q. Li, Z. Li, X. Y. Xu, Y. L. Guo, and F. Du, "Neuroprotective properties of picroside II in a rat model of focal cerebral ischemia," International Journal of Molecular Sciences, vol. 11, no. 11, pp. 4580-4590, 2010.

[307] H. Zhang, L. Zhai, T. Wang, S. Li, and Y. Guo, "Picroside II exerts a neuroprotective effect by inhibiting the mitochondria cytochrome c signal pathway following ischemia reperfusion injury in rats," Journal of Molecular Neuroscience, vol. 61, no. 2, pp. 267-278, 2017.

[308] H. R. Sadeghnia, H. Shaterzadeh, F. Forouzanfar, and H. Hosseinzadeh, "Neuroprotective effect of safranal, an active ingredient of Crocus sativus, in a rat model of transient cerebral ischemia," Folia Neuropathologica, vol. 55, no. 3, pp. 206-213, 2017. 
[309] Q. L. Zhang, B. M. Fu, and Z. J. Zhang, "Borneol, a novel agent that improves central nervous system drug delivery by enhancing blood-brain barrier permeability," Drug Delivery, vol. 24, no. 1, pp. 1037-1044, 2017.

[310] D. D. Luo, X. Y. Chen, Z. B. Zhang et al., "Different effects of (+)borneol and ()borneol on the pharmacokinetics of osthole in rats following oral administration," Molecular Medicine Reports, vol. 15, no. 6, pp. 4239-4246, 2017.

[311] F. M. Sisti, N. A. G. Dos Santos, L. do Amaral, and A. C. Dos Santos, "The neurotrophic-like effect of carvacrol: perspective for axonal and synaptic regeneration," Neurotoxicity Research, 2021.

[312] L. Tan, J. Li, Y. Wang, and R. Tan, “Anti-neuroinflammatory effect of alantolactone through the suppression of the NF- $\kappa \mathrm{B}$ and MAPK signaling pathways," Cell, vol. 8, no. 7, p. 739, 2019.

[313] Q. Jiang, R. P. Li, Y. Tang, Y. Q. Wang, C. Liu, and M. L. Guo, "Bakkenolide-IIIa protects against cerebral damage via inhibiting NF- $\kappa$ B activation," CNS Neuroscience \& Therapeutics, vol. 21, no. 12, pp. 943-952, 2015.

[314] M. Jiang, J. Li, Q. Peng et al., "Neuroprotective effects of bilobalide on cerebral ischemia and reperfusion injury are associated with inhibition of pro-inflammatory mediator production and down-regulation of JNK1/2 and p38 MAPK activation," Journal of Neuroinflammation, vol. 11, no. 1, p. 167, 2014.

[315] D. Lang, C. Kiewert, A. Mdzinarishvili et al., "Neuroprotective effects of bilobalide are accompanied by a reduction of ischemia-induced glutamate release _in vivo_," Brain Research, vol. 1425, pp. 155-163, 2011.

[316] T. M. Schwarzkopf, S. Hagl, G. P. Eckert, and J. Klein, "Neuroprotection by bilobalide in ischemia: improvement of mitochondrial function," Die Pharmazie-An International Journal of Pharmaceutical Sciences, vol. 68, pp. 584-589, 2013.

[317] Y. Zheng, Z. Wu, F. Yi et al., "By activating Akt/eNOS bilobalide B inhibits autophagy and promotes angiogenesis following focal cerebral ischemia reperfusion," Cellular Physiology and Biochemistry, vol. 47, no. 2, pp. 604-616, 2018.

[318] M. Y. D. Fernandes, M. Carmo, A. A. Fonteles et al., "(-)- $\alpha$ Bisabolol prevents neuronal damage and memory deficits through reduction of proinflammatory markers induced by permanent focal cerebral ischemia in mice," European Journal of Pharmacology, vol. 842, pp. 270-280, 2019.

[319] L. Dong, H. Qiao, X. Zhang et al., "Parthenolide Is Neuroprotective in Rat Experimental Stroke Model: Downregulating NF- B, Phospho-p38MAPK, and Caspase-1 and Ameliorating BBB Permeability," Mediators of Inflammation, vol. 2013, Article ID 370804, 10 pages, 2013.

[320] L. L. Wei, Y. Chen, Q. Y. Yu, Y. Wang, and G. Liu, "Patchouli alcohol protects against ischemia/reperfusion-induced brain injury _via_ inhibiting neuroinflammation in normal and obese mice," Brain Research, vol. 1682, pp. 61-70, 2018.

[321] X. Tian, H. Liu, F. Xiang, L. Xu, and Z. Dong, “ $\beta$-Caryophyllene protects against ischemic stroke by promoting polarization of microglia toward M2 phenotype via the TLR4 pathway," Life Sciences, vol. 237, article 116915, 2019.

[322] Z. Feng, Q. Sun, W. Chen, Y. Bai, D. Hu, and X. Xie, "The neuroprotective mechanisms of ginkgolides and bilobalide in cerebral ischemic injury: a literature review," Molecular Medicine, vol. 25, no. 1, p. 57, 2019.
[323] J. Xiang, J. Zhang, X. Cai et al., "Bilobalide protects astrocytes from oxygen and glucose deprivation-induced oxidative injury by upregulating manganese superoxide dismutase," Phytotherapy Research, vol. 33, no. 9, pp. 2329-2336, 2019.

[324] V. Madgula, B. Avula, Y. Yu et al., "Intestinal and blood-brain barrier permeability of ginkgolides and Bilobalide:In VitroandIn VivoApproaches," Planta Medica, vol. 76, no. 6, pp. 599-606, 2010.

[325] S. J. Chan, W. S. Wong, P. T. Wong, and J. S. Bian, "Neuroprotective effects of andrographolide in a rat model of permanent cerebral ischaemia," British Journal of Pharmacology, vol. 161, no. 3, pp. 668-679, 2010.

[326] C. M. Chern, K. T. Liou, Y. H. Wang, J. F. Liao, J. C. Yen, and Y. C. Shen, "Andrographolide inhibits PI3K/AKT-dependent NOX2 and iNOS expression protecting mice against hypoxia/ischemia-induced oxidative brain injury," Planta Medica, vol. 77, no. 15, pp. 1669-1679, 2011.

[327] Y. C. Hou, Y. H. Wang, K. T. Liou, and Y. C. Shen, "Neuroprotective effect of andrographolide against ischemic stroke in rats through reducing iNOS and gp91phox/NOX2 expression," Journal of Chinese Medicine, vol. 21, pp. 85-98, 2010.

[328] D. P. Wang, H. Yin, Q. Lin et al., “Andrographolide enhances hippocampal BDNF signaling and suppresses neuronal apoptosis, astroglial activation, neuroinflammation, and spatial memory deficits in a rat model of chronic cerebral hypoperfusion," Naunyn-Schmiedeberg's Archives of Pharmacology, vol. 392, no. 10, pp. 1277-1284, 2019.

[329] K. F. Lee, J. H. Chen, C. C. Teng et al., "Protective effects of Hericium erinaceus mycelium and its isolated erinacine A against ischemia-injury-induced neuronal cell death via the inhibition of iNOS/p38 MAPK and nitrotyrosine," International Journal of Molecular Sciences, vol. 15, no. 9, pp. 15073-15089, 2014.

[330] J. H. Gu, J. B. Ge, M. Li, F. Wu, W. Zhang, and Z. H. Qin, "Inhibition of NF- $\kappa \mathrm{B}$ activation is associated with antiinflammatory and anti- apoptotic effects of Ginkgolide B in a mouse model of cerebral ischemia/reperfusion injury," European Journal of Pharmaceutical Sciences, vol. 47, no. 4, pp. 652-660, 2012.

[331] P. Lv, W. Fang, X. Geng, Q. Yang, Y. Li, and L. Sha, “Therapeutic neuroprotective effects of ginkgolide $\mathrm{B}$ on cortex and basal ganglia in a rat model of transient focal ischemia," European Journal of Pharmaceutical Sciences, vol. 44, no. 3, pp. 235-240, 2011.

[332] S. Ma, H. Yin, L. Chen, H. Liu, M. Zhao, and X. Zhang, "Neuroprotective effect of ginkgolide $\mathrm{K}$ against acute ischemic stroke on middle cerebral ischemia occlusion in rats," Journal of Natural Medicines, vol. 66, no. 1, pp. 25-31, 2012.

[333] X. Niu, C. Yu, G. Jiang et al., "Pseudopterosin A ameliorates ischaemia-induced brain injury by acting on Akt signalling pathway," Folia Neuropathologica, vol. 56, no. 2, pp. 104$111,2018$.

[334] H. Dong, W. Zhou, J. Xin et al., "Salvinorin A moderates postischemic brain injury by preserving endothelial mitochondrial function _via_AMPK/Mfn2 activation," Experimental Neurology, vol. 322, article 113045, 2019.

[335] J. H. Park, O. Park, J. H. Cho et al., “Anti-inflammatory effect of tanshinone I in neuroprotection against cerebral ischemiareperfusion injury in the gerbil hippocampus," Neurochemical Research, vol. 39, no. 7, pp. 1300-1312, 2014.

[336] Y. Chen, X. Wu, S. Yu et al., "Neuroprotective capabilities of tanshinone IIA against cerebral ischemia/reperfusion injury 
via anti-apoptotic pathway in rats," Biological \& Pharmaceutical Bulletin, vol. 35, no. 2, pp. 164-170, 2012.

[337] Y. Chen, X. Wu, S. Yu et al., "Neuroprotection of tanshinone IIA against cerebral ischemia/reperfusion injury through inhibition of macrophage migration inhibitory factor in rats," PLoS One, vol. 7, no. 6, article e40165, 2012.

[338] L. Liu, X. Zhang, L. Wang et al., "The neuroprotective effects of tanshinone IIA are associated with induced nuclear translocation of TORC1 and upregulated expression of TORC1, pCREB and BDNF in the acute stage of ischemic stroke," Brain Research Bulletin, vol. 82, no. 3-4, pp. 228-233, 2010.

[339] X. Liu, C. An, P. Jin, X. Liu, and L. Wang, "Protective effects of cationic bovine serum albumin-conjugated PEGylated tanshinone IIA nanoparticles on cerebral ischemia," Biomaterials, vol. 34, no. 3, pp. 817-830, 2013.

[340] X. Liu, M. Ye, C. An, L. Pan, and L. Ji, “The effect of cationic albumin-conjugated PEGylated tanshinone IIA nanoparticles on neuronal signal pathways and neuroprotection in cerebral ischemia," Biomaterials, vol. 34, no. 28, pp. 6893-6905, 2013.

[341] Q. Tang, R. Han, H. Xiao, J. Shen, Q. Luo, and J. Li, "Neuroprotective effects of tanshinone IIA and/or tetramethylpyrazine in cerebral ischemic injury in vivo and in vitro," Brain Research, vol. 1488, pp. 81-91, 2012.

[342] J. G. Wang, S. C. Bondy, L. Zhou et al., "Protective effect of tanshinone IIA against infarct size and increased HMGB1, $\mathrm{NF} \kappa \mathrm{B}, \mathrm{GFAP}$ and apoptosis consequent to transient middle cerebral artery occlusion," Neurochemical Research, vol. 39, no. 2, pp. 295-304, 2014.

[343] L. Zhou, J. Zhang, C. Wang, and Q. Sun, “Tanshinone inhibits neuronal cell apoptosis and inflammatory response in cerebral infarction rat model," International Journal of Immunopathology and Pharmacology, vol. 30, no. 2, pp. 123-129, 2017.

[344] Y. Gao, X. Xu, S. Chang et al., “Totarol prevents neuronal injury in vitro and ameliorates brain ischemic stroke: potential roles of Akt activation and HO-1 induction," Toxicology and Applied Pharmacology, vol. 289, no. 2, pp. 142-154, 2015.

[345] S. Bai, Z. Hu, Y. Yang et al., "Anti-inflammatory and neuroprotective effects of triptolide via the NF- $\kappa \mathrm{B}$ signaling pathway in a rat MCAO model," The Anatomical Record, vol. 299, no. 2, pp. 256-266, 2016.

[346] M. Hao, X. Li, J. Feng, and N. Pan, "Triptolide protects against ischemic stroke in rats," Inflammation, vol. 38, no. 4, pp. 1617-1623, 2015.

[347] A. H. Martins, J. Hu, Z. Xu et al., "Neuroprotective activity of (1_S_,2_E_,4_R_,6_R_,-7_E_, 11_E_ )-2,7,11-cembratriene-4,6-diol (4R) _in vitro_ and _in vivo_ in rodent models of brain ischemia," Neuroscience, vol. 291, pp. 250259, 2015.

[348] T. Jayakumar, C. Y. Hsieh, J. J. Lee, and J. R. Sheu, "Experimental and clinical pharmacology of Andrographis paniculata and its major bioactive phytoconstituent andrographolide," Evidence-based Complementary and Alternative Medicine, vol. 2013, Article ID 846740, 16 pages, 2013.

[349] J. Lu, Y. Ma, J. Wu et al., "A review for the neuroprotective effects of andrographolide in the central nervous system," Biomedicine \& Pharmacotherapy, vol. 117, article 109078, 2019.

[350] J. L. Geng, J. Y. Aa, S. Q. Feng et al., "Exploring the neuroprotective effects of ginkgolides injection in a rodent model of cerebral ischemia-reperfusion injury by GC-MS based meta- bolomic profiling," Journal of Pharmaceutical and Biomedical Analysis, vol. 142, pp. 190-200, 2017.

[351] W. Zhang, J. K. Song, R. Yan et al., "Diterpene ginkgolides protect against cerebral ischemia/reperfusion damage in rats by activating Nrf2 and CREB through PI3K/Akt signaling," Acta Pharmacologica Sinica, vol. 39, no. 8, pp. 1259-1272, 2018.

[352] X. Zhou, H. Y. Wang, B. Wu et al., "Ginkgolide K attenuates neuronal injury after ischemic stroke by inhibiting mitochondrial fission and GSK-3 $\beta$-dependent increases in mitochondrial membrane permeability," Oncotarget, vol. 8, no. 27, pp. 44682-44693, 2017.

[353] Y. Zhang and J. M. Miao, "Ginkgolide K promotes astrocyte proliferation and migration after oxygen- glucose deprivation via inducing protective autophagy through the AMPK/mTOR/ULK1 signaling pathway," European Journal of Pharmacology, vol. 832, pp. 96-103, 2018.

[354] Q. Liu, Z. Jin, Z. Xu et al., “Antioxidant effects of ginkgolides and bilobalide against cerebral ischemia injury by activating the Akt/Nrf2 pathway in vitro and in vivo," Cell Stress \& Chaperones, vol. 24, no. 2, pp. 441-452, 2019.

[355] Z. Jiang, W. Gao, and L. Huang, "Tanshinones, critical pharmacological components in Salvia miltiorrhiza," Frontiers in Pharmacology, vol. 10, p. 202, 2019.

[356] S. R. Chen, Y. Dai, J. Zhao, L. Lin, Y. Wang, and Y. Wang, "A mechanistic overview of triptolide and celastrol, natural products from Tripterygium wilfordii Hook F," Frontiers in Pharmacology, vol. 9, p. 104, 2018.

[357] L. Yaidikar and S. Thakur, "Arjunolic acid, a pentacyclic triterpenoidal saponin of _Terminalia arjuna_ bark protects neurons from oxidative stress associated damage in focal cerebral ischemia and reperfusion," Pharmacological Reports, vol. 67, no. 5, pp. 890-895, 2015.

[358] K. Y. Lee, O. N. Bae, K. Serfozo et al., "Asiatic acid attenuates infarct volume, mitochondrial dysfunction, and matrix metalloproteinase-9 induction after focal cerebral ischemia," Stroke, vol. 43, no. 6, pp. 1632-1638, 2012.

[359] K. Y. Lee, O. N. Bae, S. Weinstock, M. Kassab, and A. Majid, "Neuroprotective effect of asiatic acid in rat model of focal embolic stroke," Biological \& Pharmaceutical Bulletin, vol. 37, no. 8, pp. 1397-1401, 2014.

[360] Y. Ding, M. Chen, M. Wang et al., "Neuroprotection by Acetyl-11-Keto- $\beta$-Boswellic Acid, in Ischemic Brain Injury Involves the Nrf2/HO-1 defense Pathway," Scientific Reports, vol. 4, 2015.

[361] Y. Ding, M. Chen, M. Wang, Y. Li, and A. Wen, "Posttreatment with 11-Keto- $\beta$-Boswellic acid ameliorates cerebral ischemia-reperfusion injury: $\mathrm{Nrf} 2 / \mathrm{HO}-1$ pathway as a potential mechanism," Molecular Neurobiology, vol. 52, no. 3, pp. 1430-1439, 2015.

[362] Z. Ruan, H. M. Wang, X. T. Huang et al., "A novel caffeoyl triterpene attenuates cerebral ischemic injury with potent anti-inflammatory and hypothermic effects," Journal of Neurochemistry, vol. 133, no. 1, pp. 93-103, 2015.

[363] Y. Li, D. He, X. Zhang et al., "Protective effect of celastrol in rat cerebral ischemia model: Down-regulating p-JNK, p-cJun and NF- $\kappa$ B," Brain Research, vol. 1464, pp. 8-13, 2012.

[364] H. Yu, W. Li, X. Cao et al., "Echinocystic acid, a natural plant extract, alleviates cerebral ischemia/reperfusion injury via inhibiting the JNK signaling pathway," European Journal of Pharmacology, vol. 861, article 172610, 2019. 
[365] M. N. Oztanir, O. Ciftci, A. Cetin, M. A. Durak, N. Basak, and Y. Akyuva, "The beneficial effects of $18 \beta$-glycyrrhetinic acid following oxidative and neuronal damage in brain tissue caused by global cerebral ischemia/reperfusion in a C57BL/J6 mouse model," Neurological Sciences, vol. 35, no. 8, pp. 1221-1228, 2014.

[366] Y. Qian, M. Huang, T. Guan et al., "Maslinic acid promotes synaptogenesis and axon growth via Akt/GSK-3 $\beta$ activation in cerebral ischemia model," European Journal of Pharmacology, vol. 764, pp. 298-305, 2015.

[367] L. Li, X. Zhang, L. Cui et al., "Ursolic acid promotes the neuroprotection by activating Nrf2 pathway after cerebral ischemia in mice," Brain Research, vol. 1497, pp. 32-39, 2013.

[368] Y. Luo, Y. P. Yang, J. Liu et al., "Neuroprotective effects of madecassoside against focal cerebral ischemia reperfusion injury in rats,” Brain Research, vol. 1565, pp. 37-47, 2014.

[369] Y. Li, Y. Yang, Y. Zhao et al., “Astragaloside IV reduces neuronal apoptosis and parthanatos in ischemic injury by preserving mitochondrial hexokinase-II," Free Radical Biology \& Medicine, vol. 131, pp. 251-263, 2019.

[370] J. Yang, J. Li, J. Lu, Y. Zhang, Z. Zhu, and H. Wan, "Synergistic protective effect of_astragaloside IV_ - _tetramethylpyrazine_ against cerebral ischemic-reperfusion injury induced by transient focal ischemia," Journal of Ethnopharmacology, vol. 140, no. 1, pp. 64-72, 2012.

[371] W. Barakat, N. Safwet, N. N. el-Maraghy, and M. N. Zakaria, "Candesartan and glycyrrhizin ameliorate ischemic brain damage through downregulation of the TLR signaling cascade," European Journal of Pharmacology, vol. 724, pp. 43-50, 2014.

[372] G. Gong, L. Xiang, L. Yuan et al., "Protective effect of glycyrrhizin, a direct HMGB1 inhibitor, on focal cerebral ischemia/reperfusion-induced inflammation, oxidative stress, and apoptosis in rats," PLoS One, vol. 9, no. 3, article e89450, 2014.

[373] S. W. Kim, Y. Jin, J. H. Shin et al., "Glycyrrhizic acid affords robust neuroprotection in the postischemic brain via antiinflammatory effect by inhibiting HMGB1 phosphorylation and secretion," Neurobiology of Disease, vol. 46, no. 1 , pp. 147-156, 2012.

[374] S. Z. Hou, Y. Li, X. L. Zhu, Z. Y. Wang, X. Wang, and Y. Xu, "Ameliorative effects of diammonium glycyrrhizinate on inflammation in focal cerebral ischemic-reperfusion injury," Brain Research, vol. 1447, pp. 20-27, 2012.

[375] X. Dong, L. Zheng, S. Lu, and Y. Yang, "Neuroprotective effects of pretreatment of ginsenoside $\mathrm{Rb} 1$ on severe cerebral ischemia-induced injuries in aged mice: involvement of antioxidant signaling," Geriatrics \& Gerontology International, vol. 17, no. 2, pp. 338-345, 2017.

[376] X. Q. Gao, C. X. Yang, G. J. Chen et al., “Ginsenoside Rb1 regulates the expressions of brain-derived neurotrophic factor and caspase- 3 and induces neurogenesis in rats with experimental cerebral ischemia," Journal of Ethnopharmacology, vol. 132, no. 2, pp. 393-399, 2010.

[377] G. Hu, Z. Wu, F. Yang et al., "Ginsenoside Rd blocks AIF mitochondrio-nuclear translocation and NF- $\kappa$ B nuclear accumulation by inhibiting poly(ADP-ribose) polymerase-1 after focal cerebral ischemia in rats," Neurological Sciences, vol. 34, no. 12, pp. 2101-2106, 2013.

[378] L. X. Yang, X. Zhang, and G. Zhao, "Ginsenoside Rd attenuates DNA damage by increasing expression of
DNA glycosylase endonuclease VIII-like proteins after focal cerebral ischemia," Chinese Medical Journal, vol. 129, no. 16, pp. 1955-1962, 2016.

[379] R. Ye, X. Kong, Q. Yang, Y. Zhang, J. Han, and G. Zhao, "Ginsenoside Rd attenuates redox imbalance and improves stroke outcome after focal cerebral ischemia in aged mice," Neuropharmacology, vol. 61, no. 4, pp. 815-824, 2011.

[380] R. Ye, Q. Yang, X. Kong et al., "Ginsenoside Rd attenuates early oxidative damage and sequential inflammatory response after transient focal ischemia in rats," Neurochemistry International, vol. 58, no. 3, pp. 391-398, 2011.

[381] R. Ye, X. Zhang, X. Kong et al., "Ginsenoside Rd attenuates mitochondrial dysfunction and sequential apoptosis after transient focal ischemia," Neuroscience, vol. 178, pp. 169180, 2011.

[382] G. Zhang, F. Xia, Y. Zhang et al., "Ginsenoside Rd is efficacious against acute ischemic stroke by suppressing microglial proteasome-mediated inflammation," Molecular Neurobiology, vol. 53, no. 4, pp. 2529-2540, 2016.

[383] R. Ye, X. Kong, Q. Yang et al., "Ginsenoside Rd in experimental stroke: superior neuroprotective efficacy with a wide therapeutic window," Neurotherapeutics, vol. 8, no. 3, pp. 515-525, 2011.

[384] J. Chen, X. Zhang, X. Liu et al., "Ginsenoside Rg1 promotes cerebral angiogenesis via the PI3K/Akt/mTOR signaling pathway in ischemic mice," European Journal of Pharmacology, vol. 856, article 172418, 2019.

[385] S. F. Chu, Z. Zhang, X. Zhou et al., "Ginsenoside Rg1 protects against ischemic/reperfusion-induced neuronal injury through miR-144/Nrf2/ARE pathway," Acta Pharmacologica Sinica, vol. 40, no. 1, pp. 13-25, 2019.

[386] L. Wang, H. Zhao, Z. Z. Zhai, and L. X. Qu, "Protective effect and mechanism of ginsenoside Rg1 in cerebral ischaemiareperfusion injury in mice," Biomedicine \& Pharmacotherapy, vol. 99, pp. 876-882, 2018.

[387] C. L. Xie, J. H. Li, W. W. Wang, G. Q. Zheng, and L. X. Wang, "Neuroprotective effect of ginsenoside-Rg1 on cerebral ische$\mathrm{mia}$ /reperfusion injury in rats by downregulating proteaseactivated receptor-1 expression," Life Sciences, vol. 121, pp. 145-151, 2015.

[388] T. Zheng, H. Jiang, R. Jin et al., "Ginsenoside Rg1 attenuates protein aggregation and inflammatory response following cerebral ischemia and reperfusion injury," European Journal of Pharmacology, vol. 853, pp. 65-73, 2019.

[389] Y. Zhou, H. Q. Li, L. Lu et al., "Ginsenoside Rg1 provides neuroprotection against blood brain barrier disruption and neurological injury in a rat model of cerebral ischemia/reperfusion through downregulation of aquaporin 4 expression," Phytomedicine, vol. 21, no. 7, pp. 998-1003, 2014.

[390] B. He, P. Chen, J. Yang et al., "Neuroprotective effect of 20(R)-ginsenoside $\mathrm{Rg}_{3}$ against transient focal cerebral ischemia in rats," Neuroscience Letters, vol. 526, no. 2, pp. 106$111,2012$.

[391] Y. Y. Liu, T. Y. Zhang, X. Xue et al., "Pseudoginsenoside-F11 attenuates cerebral ischemic injury by alleviating autophagic/lysosomal defects," CNS Neuroscience \& Therapeutics, vol. 23, no. 7, pp. 567-579, 2017.

[392] T. Zhang, C. Wu, X. Yang et al., "Pseudoginsenoside-F11 protects against transient cerebral ischemia injury in rats involving repressing calcium overload," Neuroscience, vol. 411, pp. 86-104, 2019. 
[393] X. Meng, M. Wang, X. Wang et al., "Suppression of NADPH oxidase-and mitochondrion-derived superoxide by notoginsenoside R1 protects against cerebral ischemia-reperfusion injury through estrogen receptor-dependent activation of Akt/Nrf2 pathways," Free Radical Research, vol. 48, no. 7, pp. 823-838, 2014.

[394] N. K. Roy, D. Parama, K. Banik et al., “An update on pharmacological potential of boswellic acids against chronic diseases," International Journal of Molecular Sciences, vol. 20, no. 17, article 4101, 2019.

[395] H. L. Wang, Q. H. Zhou, M. B. Xu, X. L. Zhou, and G. Q. Zheng, "Astragaloside IV for experimental focal cerebral ischemia: preclinical evidence and possible mechanisms," Oxidative Medicine and Cellular Longevity, vol. 2017, Article ID 8424326, 13 pages, 2017.

[396] X. Huang, N. Li, Y. Pu, T. Zhang, and B. Wang, "Neuroprotective effects of ginseng phytochemicals: recent perspectives," Molecules, vol. 24, no. 16, p. 2939, 2019.

[397] X. Liu, L. Wang, A. Wen et al., "Ginsenoside-Rd improves outcome of acute ischaemic stroke - a randomized, doubleblind, placebo-controlled, multicenter trial," European Journal of Neurology, vol. 19, no. 6, pp. 855-863, 2012.

[398] X. S. Zeng, X. S. Zhou, F. C. Luo et al., "Comparative analysis of the neuroprotective effects of ginsenosides Rg1 and Rb1 extracted from Panax notoginseng against cerebral ischemia," Canadian Journal of Physiology and Pharmacology, vol. 92, no. 2, pp. 102-108, 2014.

[399] J. Shen, Z. Zhao, W. Shang et al., "Ginsenoside Rg1 nanoparticle penetrating the blood-brain barrier to improve the cerebral function of diabetic rats complicated with cerebral infarction," International Journal of Nanomedicine, vol. 12, pp. 6477-6486, 2017.

[400] Y. P. Lu, S. Y. Liu, H. Sun, X. M. Wu, J. J. Li, and L. Zhu, "Neuroprotective effect of astaxanthin on $\mathrm{H}_{2} \mathrm{O}_{2}$-induced neurotoxicity in vitro and on focal cerebral ischemia in vivo," Brain Research, vol. 1360, pp. 40-48, 2010.

[401] Y. Nai, H. Liu, X. Bi, H. Gao, and C. Ren, "Protective effect of astaxanthin on acute cerebral infarction in rats," Human \& Experimental Toxicology, vol. 37, no. 9, pp. 929-936, 2018.

[402] L. Hu, W. Chen, F. Tian, C. Yuan, H. Wang, and H. Yue, "Neuroprotective role of fucoxanthin against cerebral ischemic/reperfusion injury through activation of Nrf2/HO-1 signaling," Biomedicine \& Pharmacotherapy, vol. 106, pp. 1484-1489, 2018.

[403] S. Y. Li, D. Yang, Z. J. Fu, T. Woo, D. Wong, and A. C. Lo, "Lutein enhances survival and reduces neuronal damage in a mouse model of ischemic stroke," Neurobiology of Disease, vol. 45, no. 1, pp. 624-632, 2012.

[404] C. Galasso, I. Orefice, P. Pellone et al., "On the neuroprotective role of Astaxanthin: new perspectives?" Marine Drugs, vol. 16, no. 8, p. 247, 2018.

[405] J. Hu, Y. Chai, Y. Wang et al., "PI3K p55 $\gamma$ promoter activity enhancement is involved in the anti-apoptotic effect of berberine against cerebral ischemia-reperfusion," European Journal of Pharmacology, vol. 674, no. 2-3, pp. 132-142, 2012.

[406] S. N. Maleki, N. Aboutaleb, and F. Souri, "Berberine confers neuroprotection in coping with focal cerebral ischemia by targeting inflammatory cytokines," Journal of Chemical Neuroanatomy, vol. 87, pp. 54-59, 2018.

[407] J. Yang, H. Yan, S. Li, and M. Zhang, "Berberine ameliorates MCAO induced cerebral ischemia/reperfusion injury via activation of the BDNF-TrkB-PI3K/Akt signaling pathway," Neurochemical Research, vol. 43, no. 3, pp. 702-710, 2018.

[408] X. Zhang, X. Zhang, C. Wang et al., "Neuroprotection of early and short-time applying berberine in the acute phase of cerebral ischemia: Up-regulated pAkt, pGSK and pCREB, downregulated NF- $\kappa$ B expression, ameliorated BBB permeability," Brain Research, vol. 1459, pp. 61-70, 2012.

[409] J. Zhu, D. Cao, C. Guo et al., "Berberine facilitates angiogenesis against ischemic stroke through modulating microglial polarization via AMPK signaling," Cellular and Molecular Neurobiology, vol. 39, no. 6, pp. 751-768, 2019.

[410] N. M. de Lima, E. O. Ferreira, M. Y. Fernandes et al., "Neuroinflammatory response to experimental stroke is inhibited by boldine," Behavioural Pharmacology, vol. 28, no. 2 and 3, pp. 223-237, 2017.

[411] M. Huang, G. Cheng, H. Tan et al., "Capsaicin protects cortical neurons against ischemia/reperfusion injury via downregulating NMDA receptors," Experimental Neurology, vol. 295, pp. 66-76, 2017.

[412] A. Janyou, P. Wicha, J. Jittiwat, A. Suksamrarn, C. Tocharus, and J. Tocharus, "Dihydrocapsaicin attenuates blood brain barrier and cerebral damage in focal cerebral ischemia/reperfusion via oxidative stress and inflammatory," Scientific Reports, vol. 7, no. 1, article 10556, 2017.

[413] D. Wu, J. Shi, O. Elmadhoun et al., "Dihydrocapsaicin (DHC) enhances the hypothermia-induced neuroprotection following ischemic stroke via PI3K/Akt regulation in rat," Brain Research, vol. 1671, pp. 18-25, 2017.

[414] Z. Cao, A. Balasubramanian, and S. Marrelli, "Pharmacologically induced hypothermia via TRPV1 channel agonism provides neuroprotection following ischemic stroke when initiated 90 min after reperfusion," American Journal of Physiology. Regulatory, Integrative and Comparative Physiology, vol. 306, no. 2, pp. R149-R156, 2014.

[415] Y. M. Ha, M. Y. Kim, M. K. Park et al., "Higenamine reduces HMGB1 during hypoxia-induced brain injury by induction of heme oxygenase-1 through PI3K/Akt/Nrf-2 signal pathways," Apoptosis, vol. 17, no. 5, pp. 463-474, 2012.

[416] Y. Z. Guan, X. D. Jin, L. X. Guan et al., "Nicotine inhibits microglial proliferation and is neuroprotective in global ischemia rats," Molecular Neurobiology, vol. 51, no. 3, pp. 1480-1488, 2015.

[417] R. Sun, Y. Song, S. Li et al., "Levo-tetrahydropalmatine attenuates neuron apoptosis induced by cerebral ischemia-reperfusion injury: involvement of c-Abl activation," Journal of Molecular Neuroscience, vol. 65, no. 3, pp. 391-399, 2018.

[418] M. Li, X. Zhang, L. Cui et al., "The neuroprotection of oxymatrine in cerebral ischemia/reperfusion is related to nuclear factor erythroid 2-related factor 2 (nrf2)-mediated antioxidant response: role of nrf2 and hemeoxygenase-1 expression," Biological \& Pharmaceutical Bulletin, vol. 34, no. 5, pp. 595-601, 2011.

[419] C. Rui, L. Yuxiang, J. Ning et al., "Anti-apoptotic and neuroprotective effects of oxysophoridine on cerebral ischemia both in vivo and in vitro," Planta Medica, vol. 79, no. 11, pp. 916-923, 2013.

[420] K. Vaibhav, P. Shrivastava, H. Javed et al., "Piperine suppresses cerebral ischemia-reperfusion-induced inflammation through the repression of COX-2, NOS-2, and NF- $\kappa$ B in middle cerebral artery occlusion rat model," Molecular and Cellular Biochemistry, vol. 367, no. 1-2, pp. 73-84, 2012. 
[421] H. Huang, R. Zhong, Z. Xia, J. Song, and L. Feng, "Neuroprotective effects of rhynchophylline against ischemic brain injury via regulation of the Akt/mTOR and TLRs signaling pathways," Molecules, vol. 19, no. 8, pp. 11196-11210, 2014.

[422] J. Qiu, M. Wang, J. Zhang et al., "The neuroprotection of sinomenine against ischemic stroke in mice by suppressing NLRP3 inflammasome via AMPK signaling," International Immunopharmacology, vol. 40, pp. 492-500, 2016.

[423] S. Yang, F. Ning, J. Li et al., "Therapeutic effect analysis of sinomenine on rat cerebral ischemia-reperfusion injury," Journal of Stroke and Cerebrovascular Diseases, vol. 25, no. 5, pp. 1263-1269, 2016.

[424] T. Sharma, V. Airao, N. Panara et al., "Solasodine protects rat brain against ischemia/reperfusion injury through its antioxidant activity," European Journal of Pharmacology, vol. 725, pp. 40-46, 2014.

[425] Z. Liu, D. He, X. Zhang et al., "Neuroprotective effect of early and short-time applying sophoridine in pMCAO rat brain: down-regulated TRAF6 and up-regulated p-ERK1/2 expression, ameliorated brain infaction and edema," Brain Research Bulletin, vol. 88, no. 4, pp. 379-384, 2012.

[426] Y. Miao, B. Wu, W. Zhang, Y. Qiu, B. Li, and X. Lu, "Neuroprotective effect of sophocarpine against transient focal cerebral ischemia via down-regulation of the acid-sensing ion channel 1 in rats," Brain Research, vol. 1382, pp. 245-251, 2011.

[427] L. Ruan, H. S. Huang, W. X. Jin, H. M. Chen, X. J. Li, and Q. J. Gong, "Tetrandrine attenuated cerebral ischemia/reperfusion injury and induced differential proteomic changes in a MCAO mice model using 2-D DIGE," Neurochemical Research, vol. 38, no. 9, pp. 1871-1879, 2013.

[428] K. Pravalika, D. Sarmah, H. Kaur et al., "Trigonelline therapy confers neuroprotection by reduced glutathione mediated myeloperoxidase expression in animal model of ischemic stroke," Life Sciences, vol. 216, pp. 49-58, 2019.

[429] H. Wang, K. Zhang, L. Zhao, J. Tang, L. Gao, and Z. Wei, "Anti-inflammatory effects of vinpocetine on the functional expression of nuclear factor-kappa B and tumor necrosis factor-alpha in a rat model of cerebral ischemia-reperfusion injury," Neuroscience Letters, vol. 566, pp. 247-251, 2014.

[430] W. N. Wu, P. F. Wu, X. L. Chen et al., "Sinomenine protects against ischaemic brain injury: involvement of co-inhibition of acid-sensing ion channel 1a and L-type calcium channels," British Journal of Pharmacology, vol. 164, no. 5, pp. 14451459, 2011.

[431] F. Zhang, C. Yan, C. Wei et al., "Vinpocetine inhibits NF- $\kappa$ BDependent inflammation in acute ischemic stroke patients," Translational Stroke Research, vol. 9, no. 2, pp. 174-184, 2018. 